\title{
An atlas of white matter anatomy, its variability, and reproducibility based on Constrained Spherical Deconvolution of diffusion MRI
}

Ahmed Radwan ${ }^{1,2}$, Stefan Sunaert ${ }^{1,2,3}$, Kurt Schilling ${ }^{4}$, Maxime Descoteaux ${ }^{5}$, Bennett A. Landman ${ }^{6}$, Mathieu Vandenbulcke ${ }^{2,7,8}$, Tom Theys ${ }^{2,9,10}$, Patrick Dupont ${ }^{2,11}$, Louise Emsell $1,2,7,8$

[1] KU Leuven, Department of Imaging and pathology, Translational MRI, Leuven, Belgium

[2] KU Leuven, Leuven Brain Institute, Department of Neurosciences, Leuven, Belgium

[3] UZ Leuven, Department of Radiology, Leuven, Belgium

[4] Department of Radiology and Radiological Sciences, Vanderbilt University Medical Center, Nashville, TN, USA

[5] SCIL, Université de Sherbrooke, Quebec, Canada

[6] Department of Electrical Engineering and Computer Science, Vanderbilt University, Nashville, TN, USA

[7] KU Leuven, Department of Neurosciences, Neuropsychiatry, Leuven, Belgium [8] University Psychiatric Center (UPC) - Leuven, Geriatric Psychiatry, Leuven, Belgium

[9] KU Leuven, Department of Neurosciences, Research Group Experimental Neurosurgery and Neuroanatomy, Leuven, Belgium

[10] Department of Neurosurgery, University Hospitals Leuven, Leuven, Belgium

[11] KU Leuven, Laboratory for Cognitive Neurology, Department of Neurosciences, Leuven, Belgium 


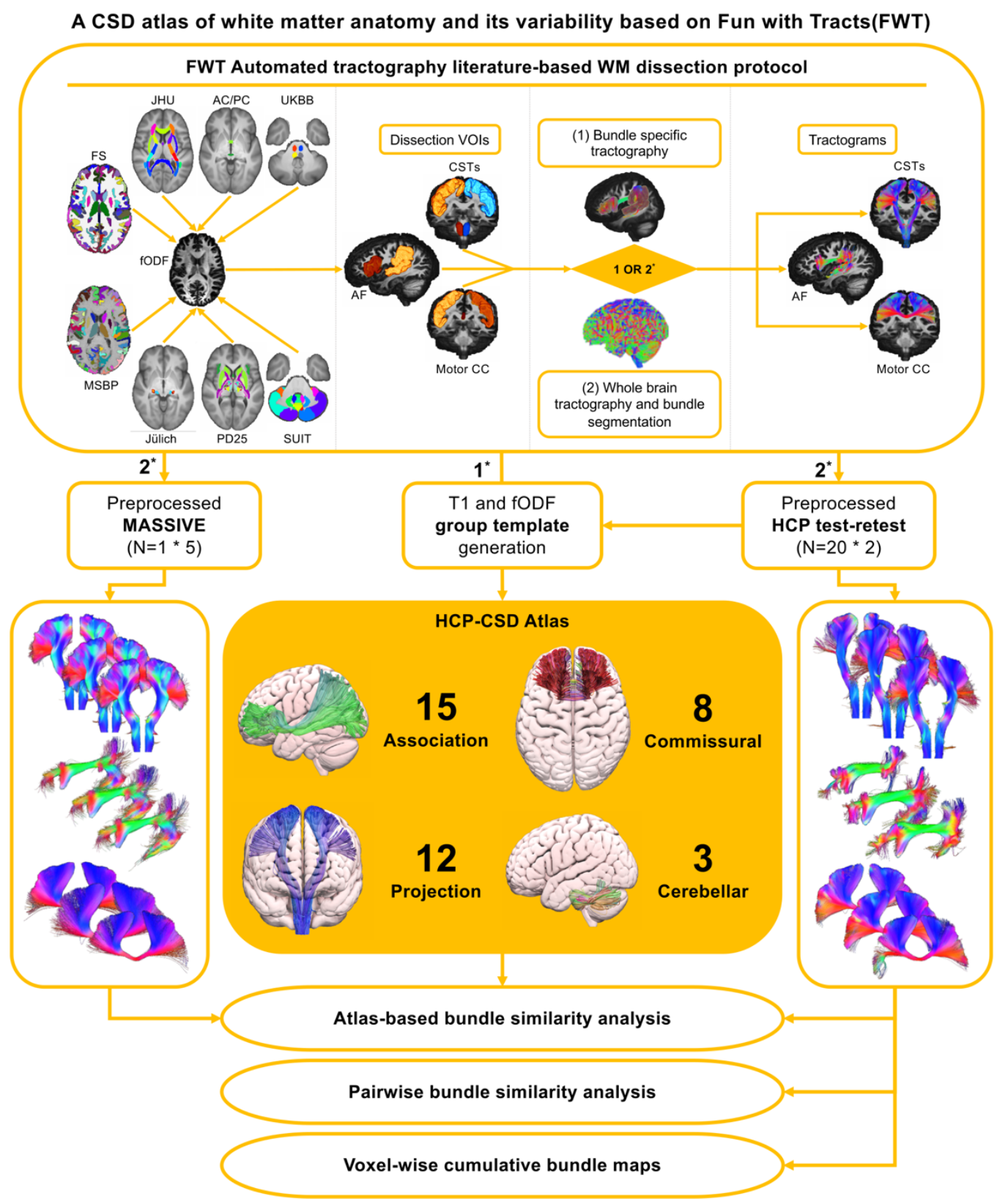

Graphical abstract: (Top) shows the FWT pipeline for both CSTs, AF, and motor CC bundles. (Left to right) show the required input structural parcellation maps and a priori atlases for FWT and the resulting virtual dissection include/exclude VOIs. FWT provides two approaches to virtual dissection: (1) is a bundle-specific approach where streamlines are only seeded for the bundle of interest, (2) is a whole brain tractography followed by streamlines segmentation, (top right) shows output tractograms. (Middle) Group-averaged T1 and fODF images are generated from the HCP testretest data, and FWT is applied to generate the HCP- atlas using the bundle-specific approach $\left(1^{*}\right)$. FWT's whole brain tracking and segmentation approach $\left(2^{*}\right)$ was applied to the HCP and MASSIVE dataset (right and left) and conducted model-based, and pair-wise similarity analyses and generated voxel-wise cumulative maps per bundle. FWT= Fun With Tracts, FS= FreeSurfer, MSBP= MultiScaleBrainParcellator, PD25= NIST Parkinson's histological, JHU= John's Hopkins university, Juelich= Juelich university histological atlas, $\mathrm{AC} / \mathrm{PC}=$ anterior commissure/posterior commissure) UKBB= UK Biobank, SUIT (spatially unbiased cerebellar atlas template), dMRI= diffusion magnetic resonance imaging, $C S D=$ constrained spherical deconvolution, $\mathrm{fODF}=$ fiber orientation distribution function, $\mathrm{CST}=$ corticospinal tract, $\mathrm{AF}=$ arcuate fasciculus, $\mathrm{CC}=$ corpus callosum, $\mathrm{HCP}=$ human connectome project, MASSIVE= Multiple acquisitions for standardization of structural imaging validation and evaluation. 


\section{Abstract}

Virtual dissection of white matter (WM) using diffusion MRI tractography is confounded by its poor reproducibility. Despite the increased adoption of advanced reconstruction models, early region-of-interest driven protocols based on diffusion tensor imaging (DTI) remain the dominant reference for virtual dissection protocols. Here we bridge this gap by providing a comprehensive description of typical WM anatomy reconstructed using a reproducible automated subject-specific parcellationbased approach based on probabilistic constrained-spherical deconvolution (CSD) tractography. We complement this with a WM template in MNI space comprising 68 bundles, including all associated anatomical tract selection labels and associated automated workflows. Additionally, we demonstrate bundle inter- and intra-subject variability using 40 (20 test-retest) datasets from the human connectome project (HCP) and 5 sessions with varying b-values and number of b-shells from the singlesubject Multiple Acquisitions for Standardization of Structural Imaging Validation and Evaluation (MASSIVE) dataset. The most reliably reconstructed bundles were the whole pyramidal tracts, primary corticospinal tracts, whole superior longitudinal fasciculi, frontal, parietal and occipital segments of the corpus callosum and middle cerebellar peduncles. More variability was found in less dense bundles, e.g., the first segment of the superior longitudinal fasciculus, fornix, dentato-rubro-thalamic tract (DRTT), and premotor pyramidal tract. Using the DRTT as an example, we show that this variability can be reduced by using a higher number of seeding attempts. Overall inter-session similarity was high for HCP test-retest data (median weighted-dice = 0.963 , stdev $=0.201$ and $I Q R=0.099)$. Compared to the HCP-template bundles there was a high level of agreement for the HCP test-retest data (median weighted-dice = 0.747 , stdev $=0.220$ and IQR $=0.277$ ) and for the MASSIVE data (median weighteddice $=0.767$, stdev $=0.255$ and $I Q R=0.338)$. In summary, this $\mathrm{WM}$ atlas provides an overview of the capabilities and limitations of automated subject-specific probabilistic CSD tractography for mapping white matter fasciculi in healthy adults. It will be most useful in applications requiring a highly reproducible parcellation-based dissection protocol, as well as being an educational resource for applied neuroimaging and clinical professionals. 


\section{Introduction}

Characterising the macroscopic structural organisation of brain connectivity in

3 vivo is central to understanding the human nervous system in health and disease. The

4 advent of diffusion magnetic resonance imaging (dMRI) fiber tractography (FT) more

5 than twenty years ago enabled significant progress in mapping major white matter

6 (WM) fiber bundles described in anatomical and surgical literature. Initial work based

7 on Diffusion Tensor Imaging (DTI) (Basser et al., 1994a, 1994b; Mori et al., 1999)

8 drove the development of white matter dissection protocols (Catani, 2006; Mori et al.,

9 2009, 2008; Wakana et al., 2007), which are still widely used today. This is because

$10 \mathrm{DTI}$ is a simple and effective approach for reconstructing the core of large fasciculi

11 (Catani et al., 2002; Stieltjes et al., 2001), data acquisition for DTI modelling requires

12 a relatively short scan time (5-10 minutes) and standard pulse sequences are widely

13 available owing to their regulatory approval for clinical practice. However, it is widely

14 accepted that DTI suffers limitations that make it suboptimal for many tractography

15 applications, particularly in a clinical setting (Farquharson et al., 2013; Mori and

16 Tournier, 2014; Tournier et al., 2011). This has led to increased interest in more

17 accurate and reliable approaches using high angular resolution imaging (HARDI) data

18 (Bayrak et al., 2020; Bloy et al., 2012; Wasserthal et al., 2018; Yeh et al., 2018). At

19 the same time, technological advances to accelerate data acquisition and

20 reconstruction, such as multiband (Bouyagoub et al., 2020; Duan et al., 2015; Larkman

21 et al., 2001; Moeller et al., 2010) and compressed sensing (Lustig et al., 2007b, 2007a)

22 in combination with improved computational efficiency, will increase the adoption of

23 advanced reconstruction models in applications traditionally reserved for DTI. This

24 means that there is a need for updated reference material based on more

25 representative virtual dissections using HARDI data acquired on 3T scanners.

26 Automated approaches relying on anatomical and orientational priors have

27 been shown to considerably improve the accuracy of tract representations (Rheault et

28 al., 2019) and address the poor reproducibility, and operator dependency that

29 confound the manual virtual dissection process (Kreilkamp et al., 2019; Maffei et al.,

30 2021; Soares et al., 2013). There are currently only a limited number of approaches

31 that automate the virtual dissection process from start to finish (Warrington et al., 2020; 
32 Wasserthal et al., 2018; Yendiki et al., 2011). However, often the underlying dissection

33 protocol is not explicitly detailed or the workflow is based on model bundles defined a

34 priori.

This work addresses both the currently unmet need for an updated HARDI

36 human white matter atlas that is relevant for clinical research studies, and a

37 standardized, reproducible virtual dissection approach based on anatomical definitions. Using CSD due to its versatility and potential application to clinical data

39 (Calamuneri et al., 2018; Toselli et al., 2017; Wilkins et al., 2015) we extend earlier

40 virtual dissection protocols based on DTI by providing a descriptive summary of the 41 normal WM anatomy of 68 fiber bundles reconstructed using probabilistic

42 tractography. We complement our anatomical descriptions with an open-source group

43 atlas in MNI space and automated subject-specific virtual dissection software, which 44 we call "Fun with Tracts" (FWT) that incorporates all the anatomical inclusion and exclusion labels per bundle. As probabilistic tractography produces inherently variable results, we also demonstrate how this may vary within and between individuals using open-source test-retest datasets from the human connectome project (HCP) (Van Essen et al., 2012) and the Multiple acquisitions for standardization of structural imaging validation and evaluation (MASSIVE) dataset (Froeling et al., 2017). The result is a detailed and accessible reference for the virtual dissection of normal white matter anatomy using CSD tractography.

\section{Material and methods}

In summary, we generated a group average template from the HCP test-retest 54 data, which was used to define the atlas of bundles based on a bundle-specific 55 seeding and tracking approach. Whole-brain tractography followed by streamlines 56 dissection was applied to the individual scans of the HCP test-retest and MASSIVE 57 datasets. Both tracking approaches used the same virtual dissection protocol.

58 Individual HCP test-retest results were used to demonstrate reproducibility in a group 59 of subjects having two scans with the same acquisition parameters. Results from the 60 MASSIVE datasets were used to demonstrate reproducibility in the same subject 61 scanned multiple times with different acquisition parameters. Further details are 62 provided below. 


\subsection{Imaging data}

\subsubsection{HCP test-retest data}

The test-retest HCP dataset (Van Essen et al., 2012) (https://db.humanconnectome.org/data/projects/HCP Retest) consists of 2 scans acquired at 1 - 11 months apart using the same scanning protocol on the same 3Tesla Siemens Skyra scanner (Siemens Healthineers, Erlangen, Germany) using a 32-channel phased array receive head coil. The HCP T1-weighted images were acquired using a 3D Magnetization Prepared Rapid Acquisition Gradient Echo (MPRAGE) pulse sequence with $0.7 \mathrm{~mm}$ isotropic voxels. The diffusion data was acquired with 90 directions per shell with b-values $\left(1000,2000, \& 3000 \mathrm{~s} / \mathrm{mm}^{2}\right)$, and $1.25 \mathrm{~mm}$ isotropic voxels. We used the preprocessed (Andersson et al., 2003a; Andersson and Sotiropoulos, 2016, 2015; Glasser et al., 2013; Jenkinson et al., 2002) imaging data of 20 random subjects (10 male / 10 female) resulting in 40 scans (see supplementary table 1 for details).

\subsubsection{MASSIVE data}

The MASSIVE dataset (Froeling et al., 2017) (http://www.massive-data.org), comprises multiple scans of the same healthy individual (female, 25 years old) using various b-values and diffusion sampling schemes. Imaging data was acquired on a 3Tesla Philips Achieva scanner (Philips Healthcare, Best, The Netherlands) with an 8channel phased array receive head coil. MASSIVE 3D T1-weighted images were acquired with $1 \mathrm{~mm}$ isotropic voxels, while dMRI images were acquired with $2.5 \mathrm{~mm}$ isotropic voxels and multiple shells with $\left(0-9000 \mathrm{~s} / \mathrm{mm}^{2}\right) \mathrm{b}$-values. We used the $(0-$ $4000 \mathrm{~s} / \mathrm{mm}^{2}$ ) b-shell data with the following b-values in s/mm² and number of diffusionweighting gradient directions respectively (b500 - 125, b1000 - 250, b2000 - 250, b3000 - 250, b4000 - 300). Five different sessions were generated using the preprocessed dMRI data acquired with an anterior-posterior (AP) phase-encoding axis and a negative gradient polarity to create 5 different sessions. Two sessions had multishell and three had single shell data with varying b-values, numbers of diffusionweighted volumes and interleaved b0 volumes. Additionally, we used corresponding reversed-phase encoded b0 images for Echo-Planar Imaging (EPI) distortion correction in FSL (Jenkinson et al., 2012). We used the MASSIVE data to investigate 
94 FWT reproducibility in the same subject with different b-values, number of diffusion directions and b-shells. Table 1 lists the b-values and total number of volumes of all dMRI data used.

\section{Table 1: Diffusion weightings and total number of volumes}

\begin{tabular}{|l|l|l|l|l|}
\hline DataldMRI parameters & Number of volumes per shell & $\begin{array}{l}\text { Diffusion weighting shell } \\
\text { (b-values in } \mathbf{s} / \mathrm{mm}^{2} \text { ) }\end{array}$ & $\begin{array}{l}\text { Total number } \\
\text { of volumes }\end{array}$ \\
\hline HCP test-retest data & $18,90,90,90$ & $0,1000,2000,3000$ & 288 \\
\hline MASSIVE datasets & 1 & $60,126,251,126$ & $0,500,1000,2000$ & 563 \\
\cline { 2 - 5 } & 2 & $60,125,251,251$ & $0,2000,3000,4000$ & 687 \\
\cline { 2 - 5 } & 3 & 30,251 & 0,1000 & 281 \\
\cline { 2 - 5 } & 4 & 30,251 & 0,2000 & 281 \\
\hline 5 & 30,251 & 0,3000 & 281 \\
\hline
\end{tabular}

\section{7}

100

101

102

103

104

105

106

107

108

109

110

111 117 et al., 2019).

We created group averaged T1-weighted images and WM fODF maps from each individual HCP dataset in MRTrix3 (Tournier et al., 2019). These will be referred to throughout this work as the HCP-template for simplicity. The resulting averaged T1weighted image was parcellated using FreeSurfer (Fischl, 2012) and MSBP (Tourbier 


\subsubsection{Virtual white matter dissection protocol}

We describe 68 WM fiber bundles using a fully automated subject-specific whole brain tractography dissection workflow called "Fun With Tracts" (FWT) based on anatomical and neuroimaging literature definitions: 15 bilateral association bundles: The arcuate fasciculus, cingulum, fornix, frontal aslant tract, Inferior frontooccipital fasciculus, inferior and middle longitudinal fasciculi, whole superior longitudinal fasciculus and its subcomponents, uncinate fasciculus, vertical occipital fasciculus. 8 commissural bundles (anterior commissure, and corpus callosum (in 7 segments). 12 bilateral projection bundles: the medial lemniscus, optic pathway, whole pyramidal tract and its subcomponents, and thalamic radiations). 3 bilateral cerebellar bundles (Dentato-rubro-thalamic tract, inferior and middle cerebellar peduncles). Definitions for each bundle are described in the results section and the inclusion/exclusion VOls are provided in the supplementary methods.

\section{2 .4 FWT}

Figure 1 provides a schematic description of the employed methods in FWT. A detailed technical description including tracking and optimization parameters is provided in supplementary methods. The automated bash workflows can be found at (https://github.com/KUL-Radneuron/KUL_FWT.git). FWT employs automated tractography using MRTrix3 (Tournier et al., 2019) v3.0.2, which is constrained by a selection of grey matter and WM 3D VOIs/parcels used to create "inclusion" and "exclusion" areas, based on the neuroanatomical literature. These VOIs/parcels are obtained from FreeSurfer (Fischl, 2012) v6 (FS), the MSBP (Tourbier et al., 2019) v1.1.1, and several a priori atlases (see supplementary materials for details), along with custom VOls manually defined in template space (the anterior commissure midline, and posterior commissure VOIs), and other custom VOIs that are generated by label propagation, (e.g., sub-segmentation of the periventricular white matter, temporal stem, insula, and superior temporal gyrus subcortical white matter). Streamlines filtering is done using ScilPy (Bore et al., 2021; "Scilpy documentation," 2021 (Bore et al., 2021; "Scilpy documentation," 2021)) v.1.1.0 and DIPY (Garyfallidis et al., 2014) tools and v1.3.0 for all bundles except the optic radiations for which we used the fiber-to-Bundle coherence (FBC) (Meesters et al., 2017; Portegies et al., 
bioRxiv preprint doi: https://doi.org/10.1101/2021.10.13.464139; this version posted October 19, 2021. The copyright holder for this preprint (which was not certified by peer review) is the author/funder, who has granted bioRxiv a license to display the preprint in perpetuity. It is made available under aCC-BY 4.0 International license.
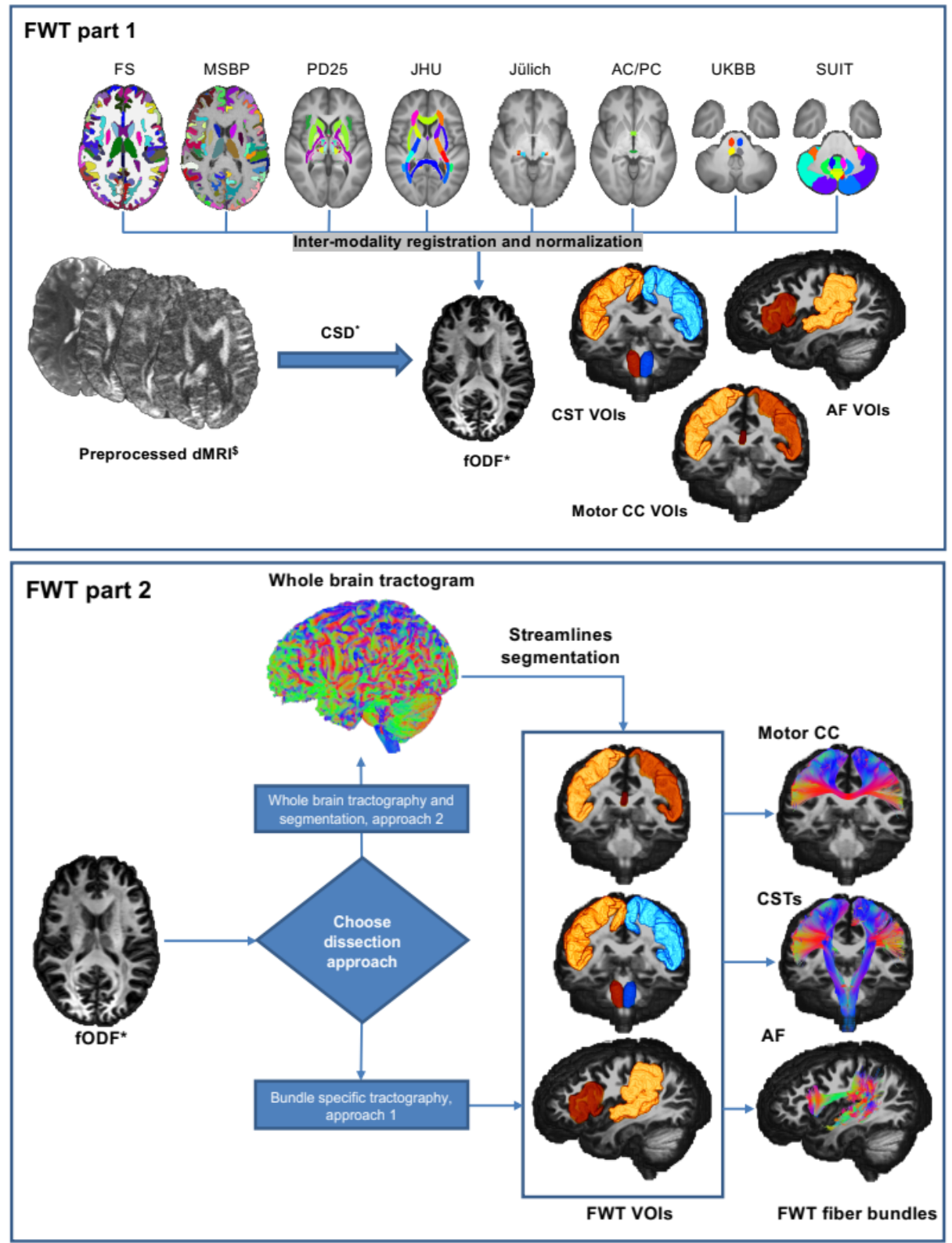

Figure 1: Graphical representation of the 2 parts of FWT for the corticospinal tracts (CSTs), arcuate fasciculi (AF), and motor corpus callosum (CC). FWT part 1 (top) requires as input preprocessed diffusion data, and FS recon-all output, and MSBP output. The script generates all VOIs used for virtual dissection by combining various anatomical VOIs from different parcellation maps and atlases. FWT part 2 (bottom) generates all tractograms from preprocessed diffusion data and VOIs created by FWT part 1, this script provides two approaches to virtual dissection; (1) Bundle specific and (2) Whole brain tractography followed by segmentation. FS = FreeSurfer, MSBP = MultiScale Brain Parcellator, PD25= NIST Parkinson's histological, $\mathrm{JHU}=$ John's Hopkins university, Juelich= Juelich university histological atlas, AC/PC $=$ anterior commissure/posterior commissure, manually defined VOIsin template space, UKBB= UK Biobank, SUIT= spatially unbiased cerebellar atlas template, $\mathrm{dMRI}=$ diffusion magnetic resonance imaging, $\mathrm{CSD}=$ constrained spherical deconvolution, fODF = fiber orientation distribution function. $\$=$ preprocessed, should include correction for motion, Eddy currents, EPI distortion, imaging noise and bias. *= Other models e.g., DTI with FACT. 


\subsubsection{Creation of inclusion and exclusion VOls}

We used the first part of the FWT workflow to automatically generate the inclusion and exclusion VOls for all bundles in every dataset. This was applied to the two sessions of each subject in the HCP test-retest dataset individually, as well as to the different scan sessions of the preprocessed MASSIVE dataset. A similar workflow designed for group template T1 images was used on the HCP-template data. FWT automatically generates VOIs from the outputs of FS (Fischl, 2012) recon-all (FreeSurferWiki, 2020), MSBP (Tourbier et al., 2019), along with the UK BioBank (UKBB) volumetric atlas of fiber bundles (Miller et al., 2016), the spatially unbiased atlas template of the cerebellum and brainstem (Diedrichsen, 2006; Diedrichsen et al., 2011, 2009; Diedrichsen and Zotow, 2015) (SUIT), the Neuroimaging and surgical technologies (NIST) Parkinson's disease histological atlas (Xiao et al., 2017, 2015, 2012) (PD25), and manually defined VOls for the anterior and posterior commissures. Finally, all resulting anatomical VOls are warped to diffusion space using ANTs (Avants et al., 2011), then combined to form bundle specific inclusion and exclusion VOIs. An equivalent workflow is available for group-averaged T1-weighted images.

\subsubsection{Individual tractography and creation of FWT-HCP template bundles}

The second part of the FWT workflow provides the choice of two different whole brain WM virtual dissection approaches, both of which rely on the inclusion and exclusion VOls created by the first part of FWT. The first approach employs bundlespecific seeding followed by streamline filtering and smoothing. The second generates a whole brain tractogram with 10 million streamlines by default followed by streamline dissection, then streamline filtering and smoothing. An equivalent workflow for fODF group averaged maps is also available generating whole brain tractograms with 20 million streamlines by default.

For this work we relied on bundle specific tractography (first approach) to generate the 68 HCP-template bundles, and whole brain tractography followed by bundle dissection (second approach) for all individual datasets. All template tractograms underwent visual quality assurance prior to further use. 


\subsubsection{Tractogram reproducilbility measures}

Tractograms can be used to generate segmentation maps and as such can be evaluated using well-known similarity/dissimilarity measures such as Hausdorff distance, overlap measures, dice similarity coefficient (DSC), etc. While image similarity metrics tend to be largely reproducible, generally intuitive and exploit the pixel or voxel-wise composition of an image, the nature of streamline data presents a particular challenge for essentially binary-based similarity measures (Rheault et al., 2020). Streamline specific variants of these measures have recently been developed but have been shown to underestimate tractogram similarity (Rheault et al., 2020). We therefore used voxel-based weighted-dice similarity (WDSC) scores to simultaneously assess overlap and streamline density agreement per voxel in our inter-subject and intra-subject variability analyses (Cousineau et al., 2017). Additional similarity measures, e.g., DSC, density correlation (Rheault et al., 2020), volume overlap and overreach (Maier-Hein et al., 2017), and bundle adjacency (Garyfallidis et al., 2012) are provided in the supplementary material. In addition, we also generated voxel-wise cumulative maps for each bundle to evaluate inter-subject variability. The equations for the different measures used, can be found in supplementary materials.

\subsection{Research questions and analyses}

(A) First, we described and presented the atlas of bundles generated with our literature-based dissection protocol (FWT) for 68 WM fasciculi based on the HCP. template data using the bundle-specific seeding and tractography approach. Additionally, we highlight inter-session variability in the HCP test-retest data with respect to the template bundles by calculating wDSC scores. These were shown in radar plots and used to calculate single-rater agreement intra-class correlation (ICC) scores.

(B) To assess inter-session/intra-subject variability in the HCP individual datasets we applied the whole-brain tractography and bundle segmentation approach. We then calculated descriptive statistics and generated violin plots for the wDSC scores resulting from simple pairwise comparisons of the output from each session. This provided a measure of FWT reproducibility within and between subjects regardless of 
210 similarity to a model bundle. HCP inter-subject variability was evaluated by generating

211 voxel-wise heat-maps of the summed binary masks of each bundle.

212 (C) FWT was applied to the MASSIVE data to test its performance on an independent 213 dataset with different acquisition parameters and sampling scheme. Model-based 214 wDSC bundle similarity scores, using the HCP-template bundles as references, were 215 then calculated for every bundle from both the HCP and MASSIVE datasets. We 216 computed wDSC descriptive statistics and generated violin plots for evaluation. This 217 provided a framework for the comparison of the outputs from both datasets. Finally, 218 we used single-rater agreement intra-class correlation scores to evaluate overall inter219 session agreement.

\section{Results}

\subsection{Qualitative bundle descriptions, template output and sample}

Detailed qualitative descriptions of the protocol used to create the inclusion and exclusion VOls for each fiber bundle along with the supporting literature are provided below. Demonstrative figures are also included for the tractography output of bundles reconstructed from the HCP-template. Bundles are grouped by type (Meynert, 1888),

227 i.e., association, commissural, and projection bundles, with the cerebellar bundles 228 grouped separately. To demonstrate inter-session variability in the source HCP test229 retest data wDSC scores for each bundle compared to HCP-template bundles are 230 shown on radar plots, and table 2 provides summary statistics for each bundle. We 231 observe that some bundles are stable and reproducible, having a high median wDSC, 232 a high minimum wDSC and a small Max.A.I.D. wDSC, while others are more variable 233 and less stable, having lower median and minimum wDSC and larger Max.A.I.D. 234 Overall single-score agreement ICC was 0.713 (upper-bound $=0.789$, lower-bound $=$ $2350.637, \mathrm{P}<0.05)$.

Table 2: Summary weighted-dice statistics for all tracked bundles derived from comparing each bundle to the corresponding HCP-template bundle

\begin{tabular}{|l|l|l|l|l|l|l|l|l|}
\hline Bundles & N & Mean & Median & STDev & Min & Max & $\begin{array}{l}\text { Max } \\
\text { A.I.D. }\end{array}$ & IQR \\
\hline AF all L/R & $40 / 40$ & $0.76 / 0.79$ & $0.80 / 0.78$ & $0.08 / 0.06$ & $0.50 / 0.65$ & $0.86 / 0.88$ & $0.03 / 0.02$ & $0.08 / 0.09$ \\
\hline CCing L/R & $40 / 40$ & $0.86 / 0.74$ & $0.86 / 0.71$ & $0.04 / 0.08$ & $0.77 / 0.59$ & $0.95 / 0.89$ & $0.03 / 0.02$ & $0.05 / 0.11$ \\
\hline
\end{tabular}




\begin{tabular}{|c|c|c|c|c|c|c|c|c|}
\hline TCing L/R & $40 / 40$ & $0.71 / 0.77$ & $0.77 / 0.82$ & $0.14 / 0.09$ & $0.17 / 0.58$ & $0.86 / 0.88$ & $0.03 / 0.03$ & $0.16 / 0.15$ \\
\hline FAT L/R & $40 / 40$ & $0.73 / 0.74$ & $0.80 / 0.79$ & $0.16 / 0.12$ & $0.25 / 0.52$ & $0.89 / 0.9$ & $0.11 / 0.13$ & $0.19 / 0.21$ \\
\hline Fx L/R & $32 / 33$ & $0.68 / 0.67$ & $0.76 / 0.73$ & $0.22 / 0.19$ & $0.13 / 0.23$ & 0.89/0.91 & $0.16 / 0.03$ & $0.28 / 0.26$ \\
\hline IFOF L/R & $40 / 40$ & $0.74 / 0.80$ & $0.77 / 0.80$ & $0.11 / 0.03$ & $0.31 / 0.70$ & $0.84 / 0.84$ & $0.10 / 0.06$ & $0.09 / 0.04$ \\
\hline ILF L/R & $40 / 40$ & $0.70 / 0.71$ & $0.7 / 0.71$ & $0.03 / 0.04$ & $0.64 / 0.61$ & $0.76 / 0.78$ & $0.05 / 0.04$ & $0.04 / 0.04$ \\
\hline IdLF L/R & $40 / 40$ & $0.73 / 0.53$ & $0.79 / 0.56$ & $0.17 / 0.15$ & $0.16 / 0.05$ & $0.89 / 0.79$ & $0.52 / 0.35$ & $0.14 / 0.18$ \\
\hline SLF all L/R & $40 / 40$ & $0.74 / 0.8$ & $0.78 / 0.81$ & $0.11 / 0.06$ & $0.35 / 0.67$ & 0.89/0.88 & $0.28 / 0.18$ & $0.09 / 0.10$ \\
\hline SLF I L/R & $40 / 40$ & $0.61 / 0.14$ & $0.67 / 0.11$ & $0.19 / 0.14$ & $0.10 / 0.00$ & $0.83 / 0.58$ & $0.10 / 0.05$ & $0.16 / 014$ \\
\hline SLF IId L/R & $40 / 40$ & $0.75 / 0.76$ & $0.79 / 0.78$ & $0.13 / 0.11$ & $0.33 / 0.40$ & $0.93 / 0.87$ & $0.2 / 0.11$ & $0.12 / 0.11$ \\
\hline SLF IIv L/R & $40 / 40$ & $0.48 / 0.52$ & $0.46 / 0.52$ & $0.22 / 0.14$ & $0.02 / 0.16$ & $0.81 / 0.75$ & $0.06 / 0.04$ & $0.33 / 0.22$ \\
\hline SLF III L/R & $40 / 40$ & $0.52 / 0.66$ & $0.53 / 0.69$ & $0.14 / 0.10$ & $0.16 / 0.41$ & $0.78 / 0.81$ & $0.03 / 0.05$ & $0.19 / 0.14$ \\
\hline UF L/R & $40 / 40$ & $0.39 / 0.50$ & $0.39 / 0.47$ & $0.08 / 0.08$ & $0.2 / 0.37$ & $0.57 / 0.64$ & $0.31 / 0.19$ & $0.10 / 0.12$ \\
\hline VOF L/R & $40 / 40$ & $0.62 / 0.28$ & $0.7 / 0.28$ & $0.19 / 0.09$ & $0 / 0.08$ & $0.80 / 0.44$ & $0.27 / 0.36$ & $0.20 / 0.15$ \\
\hline Ant Comm & 38 & 0.52 & 0.53 & 0.10 & 0.24 & 0.66 & 0.05 & 0.09 \\
\hline CC Motor & 40 & 0.67 & 0.71 & 0.16 & 0.02 & 0.84 & 0.11 & 0.10 \\
\hline CC Occipital & 40 & 0.80 & 0.82 & 0.06 & 0.64 & 0.89 & 0.49 & 0.05 \\
\hline CC Parietal & 40 & 0.84 & 0.85 & 0.03 & 0.74 & 0.90 & 0.30 & 0.04 \\
\hline $\begin{array}{l}\text { CC } \\
\text { PMCandSMA }\end{array}$ & 40 & 0.73 & 0.73 & 0.04 & 0.64 & 0.79 & 0.17 & 0.05 \\
\hline CC PreF & 40 & 0.80 & 0.80 & 0.02 & 0.75 & 0.83 & 0.20 & 0.02 \\
\hline CC Sensory & 36 & 0.72 & 0.79 & 0.19 & 0.05 & 0.90 & 0.14 & 0.20 \\
\hline CC Temporal & 39 & 0.33 & 0.37 & 0.15 & 0.02 & 0.53 & 0.03 & 0.20 \\
\hline
\end{tabular}

\begin{tabular}{lll|l|l|l|l|l|l|l}
\hline ML L/R & $40 / 40$ & $0.92 / 0.94$ & $0.94 / 0.94$ & $0.06 / 0.03$ & $0.70 / 0.85$ & $0.97 / 0.97$ & $0.46 / 0.20$ & $0.04 / 0.02$
\end{tabular}
\begin{tabular}{|l|l|l|l|l|l|l|l|l|l|}
\hline OR L/R & $40 / 40$ & $0.48 / 0.48$ & $0.55 / 0.51$ & $0.23 / 0.16$ & $0.02 / 0.11$ & $0.83 / 0.70$ & $0.11 / 0.06$ & $0.34 / 0.24$ \\
\hline
\end{tabular} \begin{tabular}{|l|l|l|l|l|l|l|l|l|l}
\hline OR OL L/R & $40 / 40$ & $0.84 / 0.83$ & $0.85 / 0.84$ & $0.04 / 0.05$ & $0.74 / 0.73$ & $0.89 / 0.93$ & $0.11 / 0.05$ & $0.05 / 0.04$ \\
\hline
\end{tabular} \begin{tabular}{|l|l|l|l|l|l|l|l|l|}
\hline OT L/R & $37 / 40$ & $0.89 / 0.87$ & $0.92 / 0.90$ & $0.09 / 0.08$ & $0.49 / 0.52$ & $0.96 / 0.95$ & $0.09 / 0.09$ & $0.06 / 0.04$ \\
\hline
\end{tabular} \begin{tabular}{|l|l|l|l|l|l|l|l|l|}
\hline PyT all L/R & $40 / 40$ & $0.91 / 0.90$ & $0.91 / 0.90$ & $0.02 / 0.01$ & $0.86 / 0.86$ & $0.94 / 0.92$ & $0.07 / 0.08$ & $0.02 / 0.01$ \\
\hline
\end{tabular} \begin{tabular}{l|l|l|l|l|l|l|l|l|l|l|}
\hline CST L/R & $40 / 40$ & $0.94 / 0.94$ & $0.94 / 0.94$ & $0.02 / 0.01$ & $0.90 / 0.91$ & $0.96 / 0.96$ & $0.45 / 0.19$ & $0.03 / 0.01$ \\
\hline
\end{tabular} \begin{tabular}{|l|l|l|l|l|l|l|l|l|l|}
\hline M1 CST L/R & $40 / 40$ & $0.94 / 0.94$ & $0.94 / 0.95$ & $0.02 / 0.01$ & $0.87 / 0.91$ & $0.96 / 0.96$ & $0.59 / 0.28$ & $0.03 / 0.02$ \\
\hline
\end{tabular} \begin{tabular}{|l|l|l|l|l|l|l|l|l|l|l|l|l|l}
\hline PyT PMC L/R & $39 / 35$ & $0.22 / 0.20$ & $0.22 / 0.21$ & $0.04 / 0.05$ & $0.11 / 0.11$ & $0.31 / 0.33$ & $0.03 / 0.06$ & $0.05 / 0.06$ \\
\hline
\end{tabular} \begin{tabular}{|l|l|l|l|l|l|l|l|l|l|}
\hline PyT SMA L/R & $40 / 40$ & $0.88 / 0.9$ & $0.90 / 0.91$ & $0.06 / 0.03$ & $0.62 / 0.79$ & $0.93 / 0.95$ & $0.40 / 0.25$ & $0.05 / 0.04$ \\
\hline
\end{tabular} \begin{tabular}{|l|l|l|l|l|l|l|l|l|l|}
\hline ATR L/R & $40 / 40$ & $0.62 / 0.56$ & $0.66 / 0.61$ & $0.19 / 0.19$ & $0.17 / 0.07$ & $0.88 / 0.84$ & $0.43 / 0.37$ & $0.24 / 0.31$ \\
\hline
\end{tabular} \begin{tabular}{|l|l|l|l|l|l|l|l|l|l}
\hline PaTR L/R & $40 / 40$ & $0.67 / 0.65$ & $0.68 / 0.67$ & $0.10 / 0.08$ & $0.42 / 0.47$ & $0.82 / 0.76$ & $0.14 / 0.14$ & $0.13 / 0.10$ \\
\hline
\end{tabular} \begin{tabular}{|l|l|l|l|l|l|l|l|l|l|}
\hline STR L/R & $40 / 40$ & $0.86 / 0.85$ & $0.87 / 0.85$ & $0.05 / 0.04$ & $0.73 / 0.70$ & $0.91 / 0.90$ & $0.10 / 0.15$ & $0.05 / 0.04$ \\
\hline
\end{tabular} \begin{tabular}{|l|l|l|l|l|l|l|l|l|l}
\hline DRTT L/R & $36 / 31$ & $0.52 / 0.46$ & $0.54 / 0.49$ & $0.18 / 0.10$ & $0.08 / 0.13$ & $0.82 / 0.60$ & $0.06 / 0.59$ & $0.22 / 0.11$ \\
\hline
\end{tabular} \begin{tabular}{|l|l|l|l|l|l|l|l|l|}
\hline ICP L/R & $40 / 40$ & $0.53 / 0.48$ & $0.57 / 0.52$ & $0.18 / 0.16$ & $0.04 / 0$ & $0.76 / 0.73$ & $0.22 / 0.40$ & $0.15 / 0.16$ \\
\hline
\end{tabular} \begin{tabular}{|l|l|l|l|l|l|l|l|l|l|l}
\hline MCP L/R & $40 / 40$ & $0.73 / 0.77$ & $0.74 / 0.77$ & $0.04 / 0.04$ & $0.63 / 0.64$ & $0.81 / 0.84$ & $0.03 / 0.29$ & $0.04 / 0.04$
\end{tabular} $\mathrm{N}=$ number of reconstructed bundles, STDev = standard deviation, Min = minimum, Max = maximum, Max. A.I.D. = maximum absolute intersession difference, $I Q R=$ interquartile range, $L=$ left, $\mathrm{R}=$ right, $\mathrm{AF}$ all = Arcuate fasciculus (excluding the precentral gyrus), $\mathrm{CCing}=$ Cingulate cingulum, TCing $=$ Temporal cingulum, FAT $=$ frontal aslant tract, $\mathrm{Fx}=$ Fornix, IFOF $=$ inferior fronto-occipital fasciculus, ILF = inferior longitudinal fasciculus, MdLF = middle longitudinal fasciculus, SLF all = superior longitudinal fasciculus (whole bundle), SLF I $=1^{\text {st }}$ segment of SLF, SLF 
bioRxiv preprint doi: $\mathrm{https}$ //doi. org/10.1101/2021.10.13.464139; this version posted October 19, 2021. The copyright holder for this preprint (which was not certified by peer review) is the author/funder, who has granted bioRxiv a license to display the preprint in perpetuity. It is made available under aCC-BY 4.0 International license.

IId = dorsal subdivision of $2^{\text {nd }}$ segment of SLF, SLF IIv = ventral subdivision of $2^{\text {nd }}$ segment of SLF, SLF III = $3^{\text {rd }}$ segment of SLF, UF = uncinate fasciculus, VOF = vertical occipital fasciculus, $\mathrm{ML}=$ medial lemniscus, $\mathrm{OR}=$ optic radiation, $\mathrm{OR} \mathrm{OL}=$ optic radiation (using whole occipital lobe as cortical inclusion), OT = optic tract, PyT all = pyramidal tract (whole bundle), CST = corticospinal tract (primary motor and primary sensory as cortical inclusion), M1 CST = corticospinal tract (only primary motor as cortical inclusion), PyT PMC = Premotor cortex pyramidal tract, PyT SMA = supplementary motor area pyramidal tract, $\mathrm{ATR}=$ anterior thalamic radiation, $\mathrm{PaTR}=$ parietal thalamic radiation, STR = superior thalamic radiation, Ant Comm = anterior commissure, $\mathrm{CC}=$ corpus callosum, CC PMCandSMA = premotor and supplementary motor corpus callosum, $\mathrm{CC}$ PreF = prefrontal corpus callosum, DRT = dentato-rubro-thalamic tract, ICP $=$ inferior cerebellar peduncle, $\mathrm{MCP}=$ middle cerebellar peduncle.

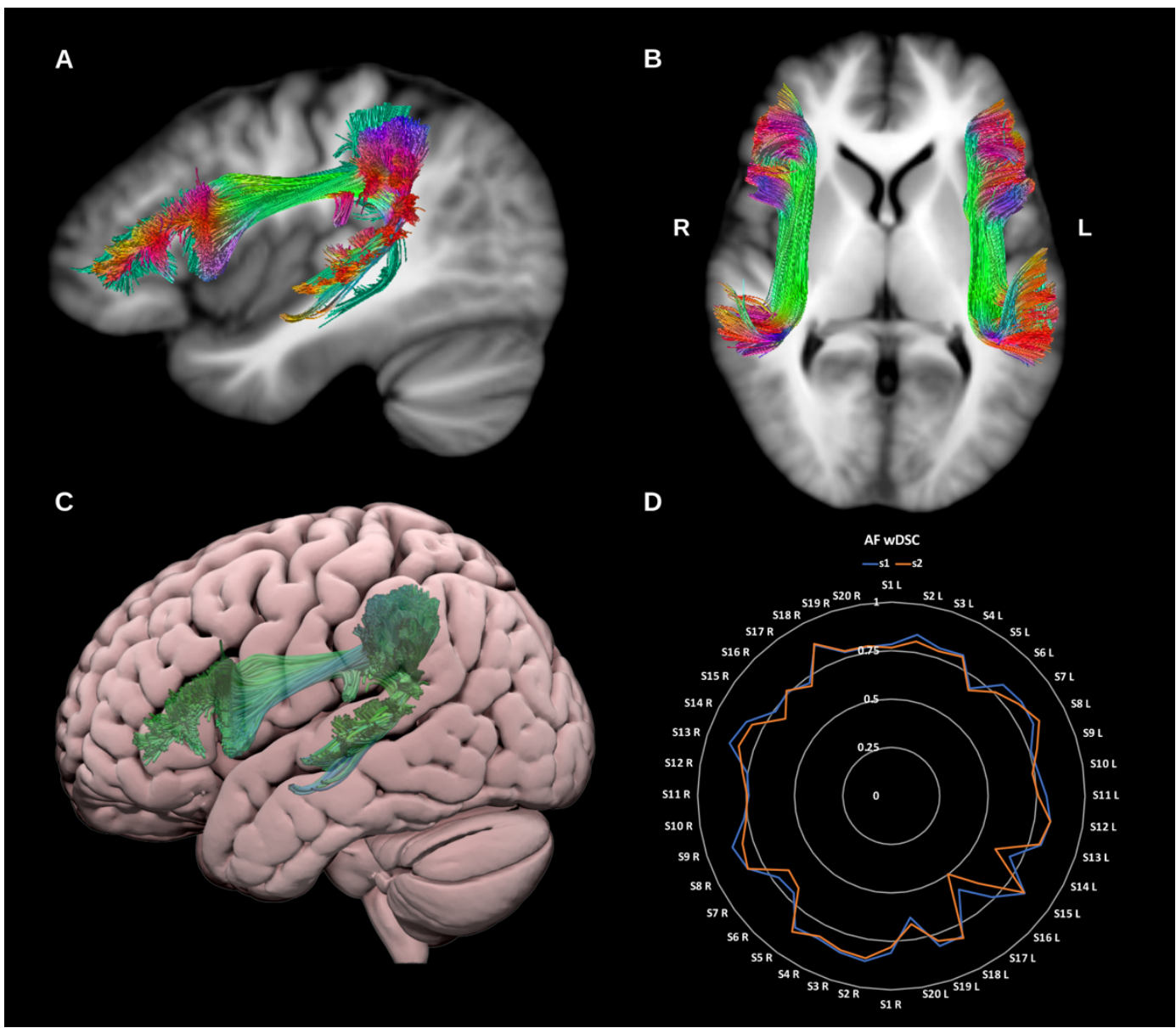

Figure 2: $(A)$ and $(B)$ show sagittal and axial slices of the T1-weighted group average image with the arcuate fasciculi overlaid in directional color coding. (C) shows a lateral projection of the semitransparent MNI pial surface with the left arcuate fasciculus in green. (D) shows a radar plot of the wDSC scores (vertical range) of both AFs using first session (blue) and second session (orange). Left $(L) A F$ 's are on the first half of the circle and right $(R)$ ones are on the second half. AF = arcuate fasciculus. $\mathrm{MNI}=$ Montreal Neurological Institute, $\mathrm{WDSC}=$ weighted dice similarity coefficient.

The AF is one of the main components of the dorsal language stream and 
240 association fiber system connecting the ventral precentral and posterior portion of the

241 inferior and middle frontal gyri with the middle and superior temporal gyri, and two

242 shorter 'in-direct' bundles: (1) an anterior network connecting the supramarginal and

243 superior temporal gyri with the precentral gyrus, and (2) a posterior network

244 connecting the posterior middle temporal gyrus with the angular gyrus (Bain et al.,

245 2019; Bernard et al., 2019; Catani, 2006; Chen et al., 2015; Fernández-Miranda et al.,

246 2015; Wakana et al., 2007; Wang et al., 2016; Yeh et al., 2018), see figure 2. The AF

247 in our data was generated for all datasets on both sides.

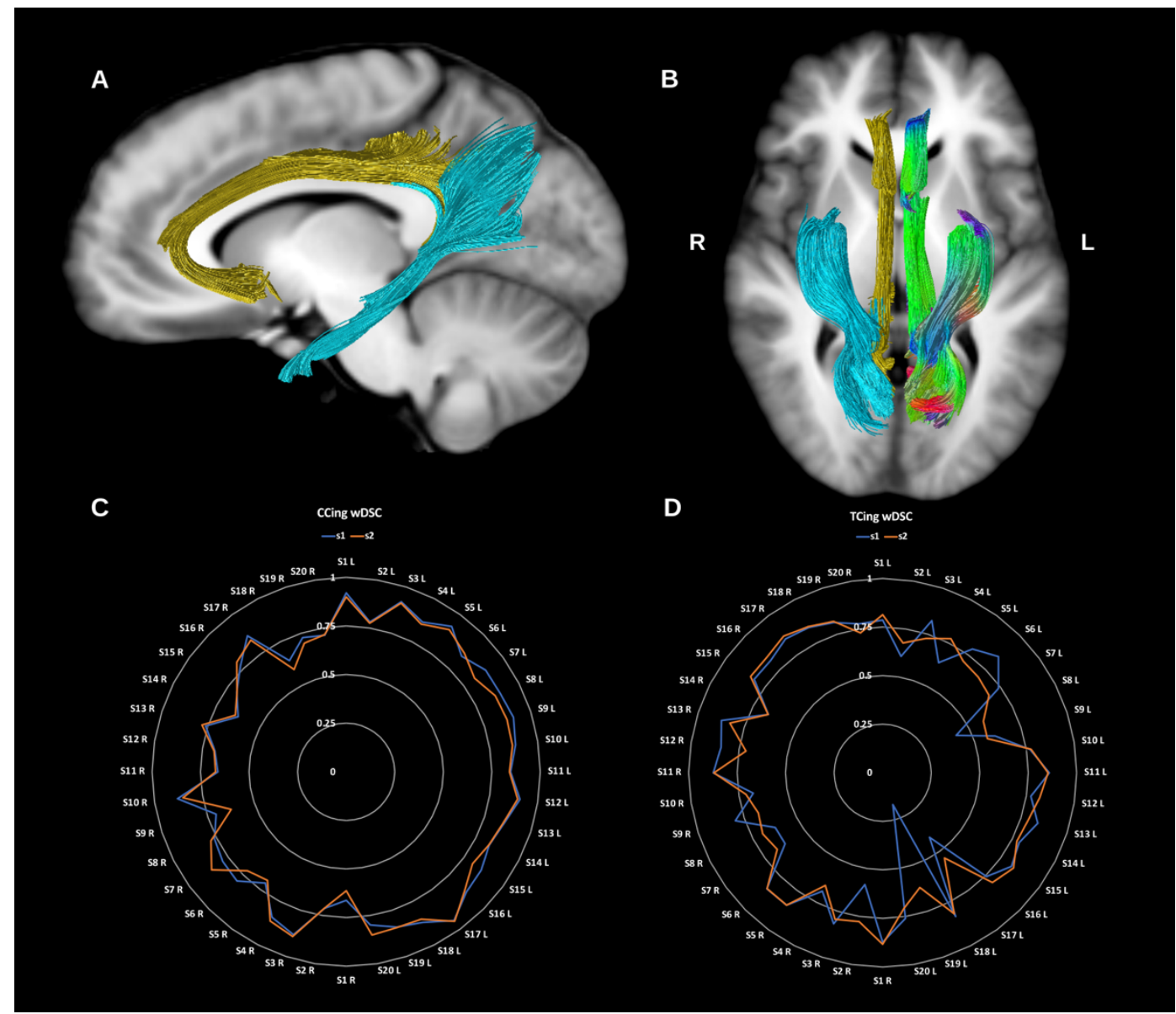

Figure 3: $(A)$ and $(B)$ show sagittal and axial slices of the T1-weighted group average image with the cingulum bundles overlaid in directional color coding for the left side and in gold for the cingulate portion (CCing) and light blue for the temporal portion (TCing). (C) and (D) show radar plots of the wDSC scores (vertical ranges) of both the CCings and TCings as reconstructed using first session (blue) and second session (orange). Left sided bundles (L) are on the first half of the circle and right ones $(\mathrm{R})$ are on the second half. $\mathrm{wDSC}=$ weighted dice similarity coefficient. 
The cingulum bundle $(\mathrm{CG})$ is the principal WM tract of the cingulate gyrus and the limbic system and is involved in a diverse range of functions spanning emotional,

252 behavioral and sensorimotor control, mnemonic processing, nociception and

253 executive function. Broadly speaking, the cingulum is a bidirectional fiber system that

254 encircles the corpus callosum lateral to the cingulate gyrus, extending from the frontal

255 lobes to the WM of the ventral temporal lobe with numerous lateral projections joining

256 and leaving the bundle along its path (Jones et al., 2013; Metzler-Baddeley et al.,

257 2012; Pascalau et al., 2018; Wu et al., 2016b), see figure 3. The complexity of

258 cingulum connections is inadequately characterized by $\mathrm{dMRI}$, which tends to

259 reconstruct either a single unilateral bundle extending from the subgenual cingulate to

260 the temporal lobe, or two subdivisions encompassing the subgenual-dorsal, and

261 dorsal-temporal segments. However, primate tracer studies suggest the CG could be

262 subdivided into three or four regions based on differences in anatomical connectivity

263 and neurotransmitter profile (Heilbronner and Haber, 2014). Here we reconstruct two

264 sub-divisions in keeping with previous dMRI literature.

\section{3.1.1.2.1 Cingulate Cingulum}

266 This represents the dorsal (cingulate) component of the cingulum and is

267 reconstructed by tracking the streamlines between the rostral and caudal anterior

268 cingulate cortex and the posterior cingulate, isthmic posterior cingulate cortex and the

269 precuneus. It is immediately superior to the body of the corpus callosum. It has a low

270 curvature $\mathrm{C}$ shape, tipped on its open end with anterior/ventral extension to the

271 subgenual cortex and ends posteriorly behind the splenium of the corpus callosum.

272 The cingulate cingulum is generated for all datasets on both sides.

\section{3.1.1.2.2 Temporal Cingullum}

274 This represents the ventral component of the cingulum and is reconstructed by

275 tracking the streamlines between the hippocampus and the isthmic posterior cingulate

276 cortex and precuneus posteriorly. It is located in the medial temporal lobe, and has a

277 slanted orientation, ascending from the anterior medial temporal lobe to the midline

278 parietal region, behind the splenium of the corpus callosum. The temporal cingulum is

279 generated for all datasets on both sides but was less reproducible than the cingulate 280 cingulum. 


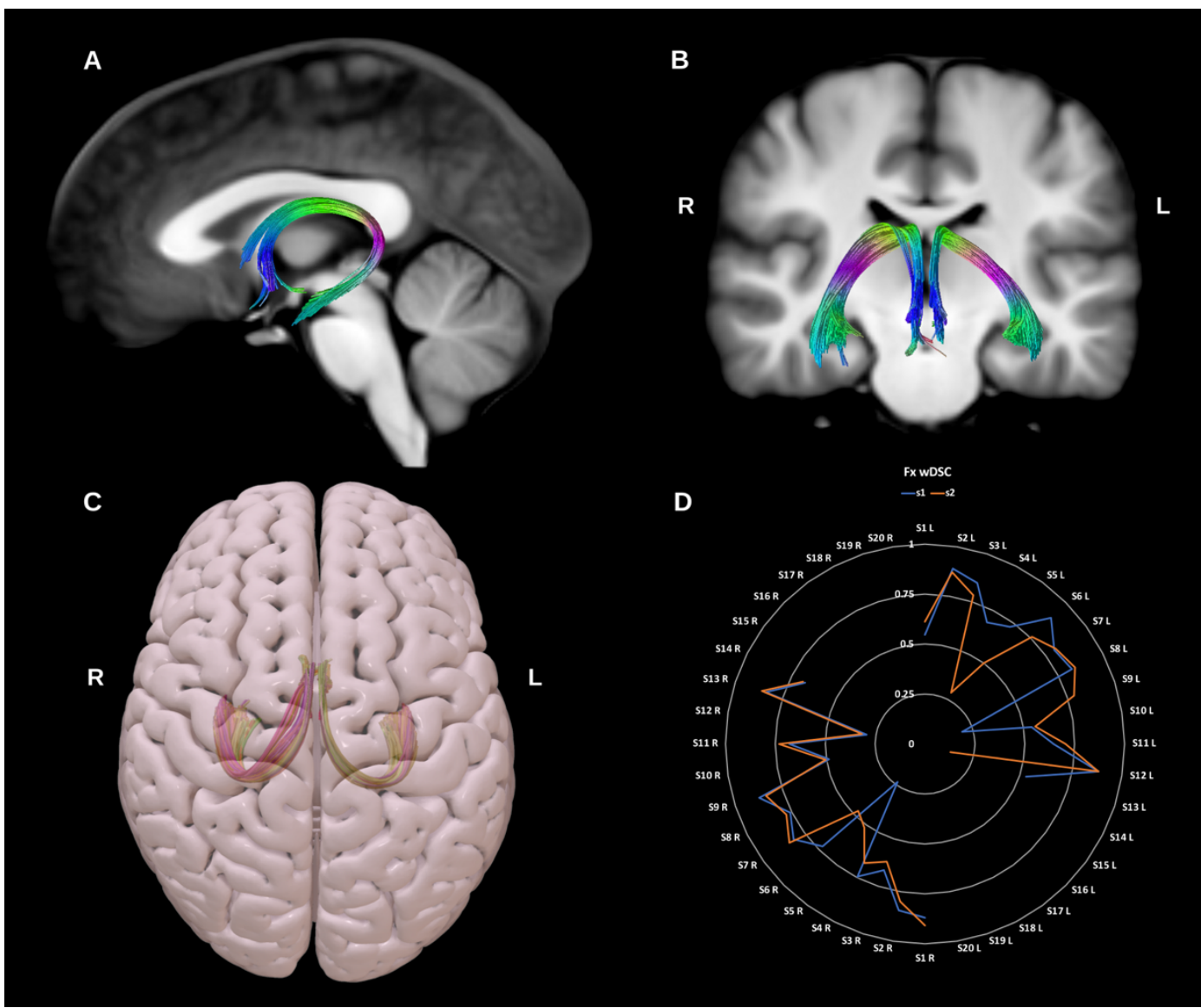

Figure 4: $(A)$ and $(B)$ show sagittal and coronal slices of the T1-weighted group average image with the fornix (Fx) overlaid in directional color coding. (C) shows a superior projection of the semitransparent MNI pial surface with both fornices shown in yellow and red. (D) shows a radar plot of the wDSC scores (vertical range) of both fornices as reconstructed using first session (blue) and second session (orange) data. A missing line indicates a missing bundle for that dataset. Left sided bundles $(L)$ are on the first half of the circle and right ones $(R)$ are on the second half. $M N I=$ Montreal Neurological Institute, wDSC = weighted dice similarity coefficient.

The fornix is the major fiber pathway associated with the hippocampus, and comprises predominantly efferent fibers connecting the hippocampus with the prefrontal cortex, the anterior thalamic nuclei, the mammillary bodies, the ventral striatum, and the basal forebrain. Initially formed by the alveus and fimbria, WM of the bilateral hippocampus coalesce as the fornix crus and body before diverging again into the pre-commissural and post-commissural fornix columns which derive their name from their position relative to the anterior commissure. Recent research suggests fibers within the fornix are arranged topographically reflecting functional 290 anterior-posterior gradient along the long axis of the hippocampus, with laterally 291 located fibers arising from the anterior hippocampus and medially located fibers originating in the posterior hippocampus (Christiansen et al., 2017; Liacu et al., 2012; 
bioRxiv preprint doi: https://doi.org/10.1101/2021.10.13.464139; this version posted October 19, 2021. The copyright holder for this preprint (which was not certified by peer review) is the author/funder, who has granted bioRxiv a license to display the preprint in perpetuity. It is made available under aCC-BY 4.0 International license.

293 Pascalau et al., 2018; Strange et al., 2014), see figure 4. Here we reconstruct the 294 fornix as a single lateralized bundle for each side. Reproducibility was poor and reconstruction failed in 15 datasets.

\subsubsection{Frontal aslant tract (FAT)}

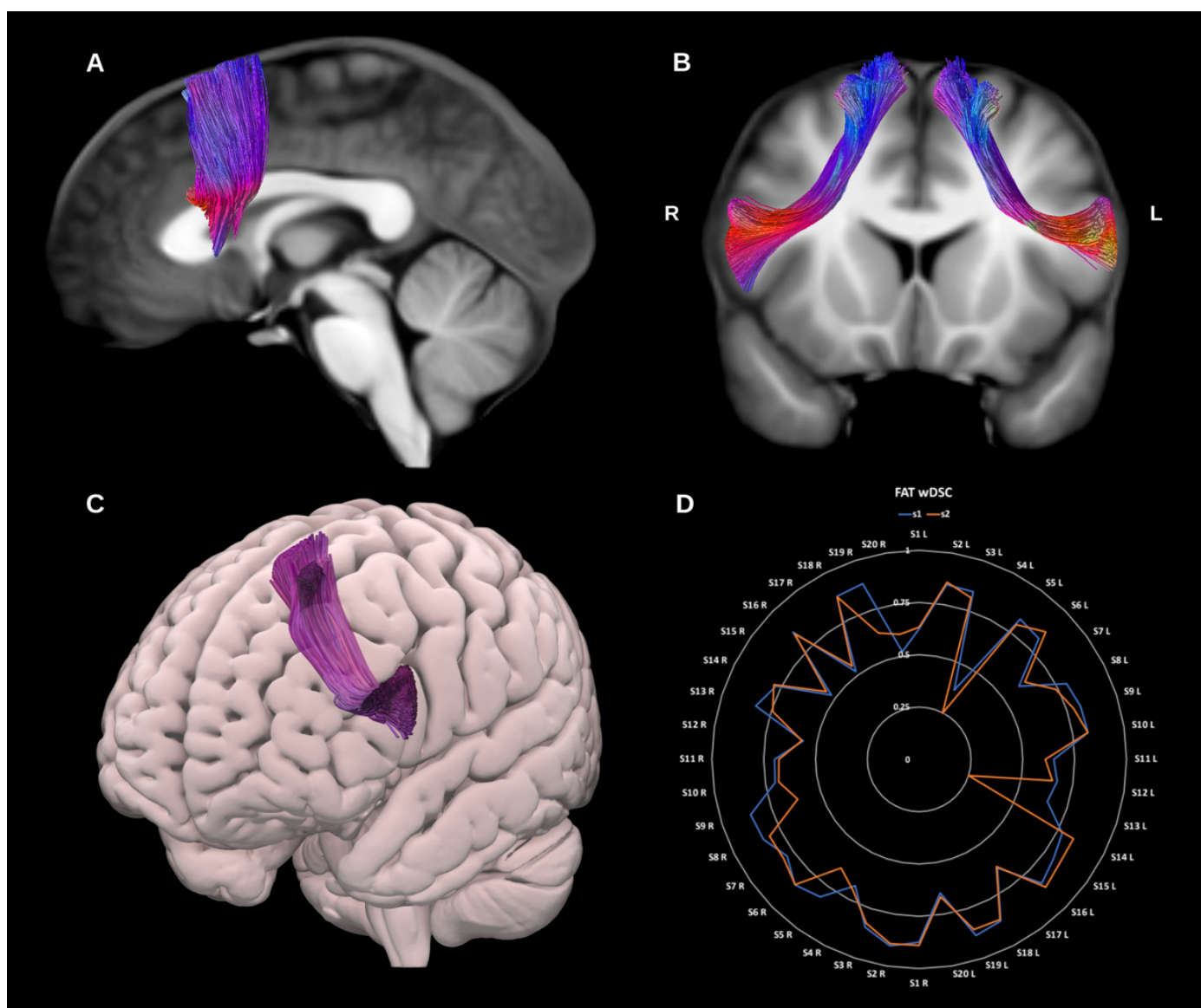

Figure 5: $(A)$ and $(B)$ show sagittal and coronal slices of the T1-weighted group average image with the frontal aslant tracts (FAT) overlaid in directional color coding. (C) shows an oblique anterior projection of the semitransparent MNI pial surface with left FAT in purple. (D) shows a radar plot of the wDSC scores (vertical range) of both FATs as reconstructed using first session (blue) and second session (orange) data. Left sided bundles $(L)$ are on the first half of the circle and right ones $(R)$ are on the second half. MNI = Montreal Neurological Institute, wDSC $=$ weighted dice similarity coefficient.

The FAT is a recently described association bundle connecting the inferior and superior frontal lobe, and is involved in speech initiation, verbal fluency and executive

299 function/inhibitory control. More specifically it connects the pars opercularis and pars

300 triangularis in the inferior frontal gyrus (IFG) with the pre-supplementary motor area 301 (pre-SMA), supplementary motor area (SMA), and the anterior cingulate cortex (Dick 302 et al., 2019; La Corte et al., 2021; Pascual-Diaz et al., 2020), see figure 5. The FAT was successfully reconstructed for all datasets on both sides. 


\subsubsection{Inferior Fronto-occipital fasciculus (IFOF)}

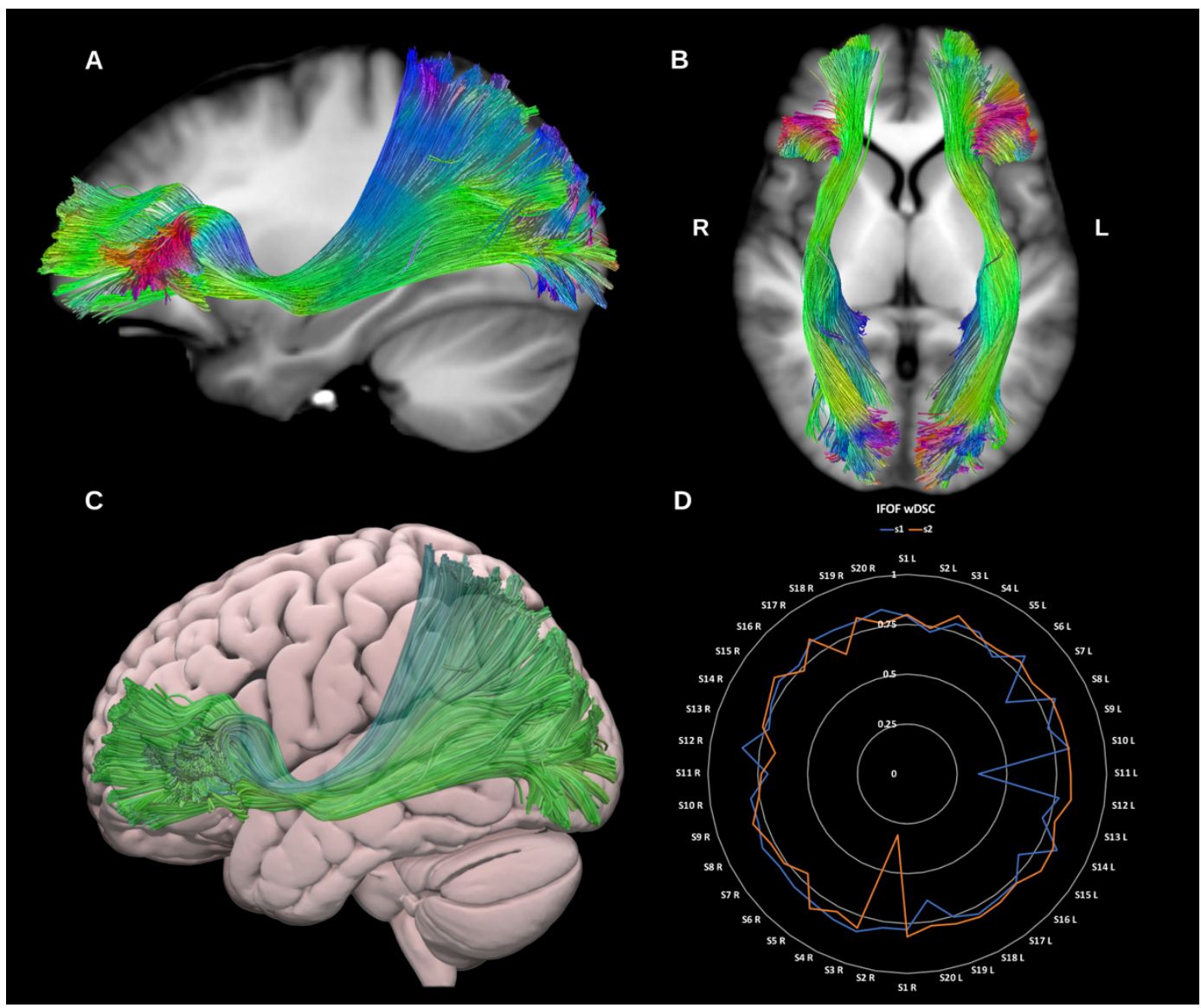

Figure 6: $(A)$ and $(B)$ show sagittal and axial slices of the T1-weighted group average image with the inferior fronto-occipital fasciculi (IFOF) overlaid in directional color coding. (C) shows a lateral projection of the semitransparent MNI pial surface with left IFOF in green. (D) shows a radar plot of the wDSC scores (vertical range) of both IFOFs as reconstructed using first session (blue) and second session (orange) data. Left sided bundles $(L)$ are on the first half of the circle and right ones $(\mathrm{R})$ are on the second half. MNI = Montreal Neurological Institute, $\mathrm{wDSC}=$ weighted dice similarity coefficient.

The IFOF is a large, long-range association fiber bundle connecting the occipital

and temporal lobes to the frontal lobes, specifically the lingual, posterior fusiform, cuneus and polar occipital cortex, with the inferior frontal gyrus, medial fronto-orbital region and frontal pole, see figure 6 . Notably, it narrows at the level of the extreme capsule. Though theoretically distinct from other temporal lobe association pathways,

310 the IFOF runs in close proximity to the middle longitudinal fasciculus (MdLF), inferior 311 longitudinal fasciculus (ILF) and uncinate fasciculus (UF), which may pose issues for

312 some tracking algorithms (Forkel et al., 2014; Wu et al., 2016a). Considerable debate 313 prevails regarding its exact functions however it is believed to serve the ventral visual 314 and language streams along with the inferior longitudinal fasciculus (ILF) and uncinate 
315 fasciculus (UF) (Caverzasi et al., 2016). The right IFOF may be related to facial 316 recognition functions and semantic visual stream processes (Herbet et al., 2018),

317 while the left IFOF is related to semantic language functions (Almairac et al., 2015).

318 The IFOF was generated for all datasets on both sides.

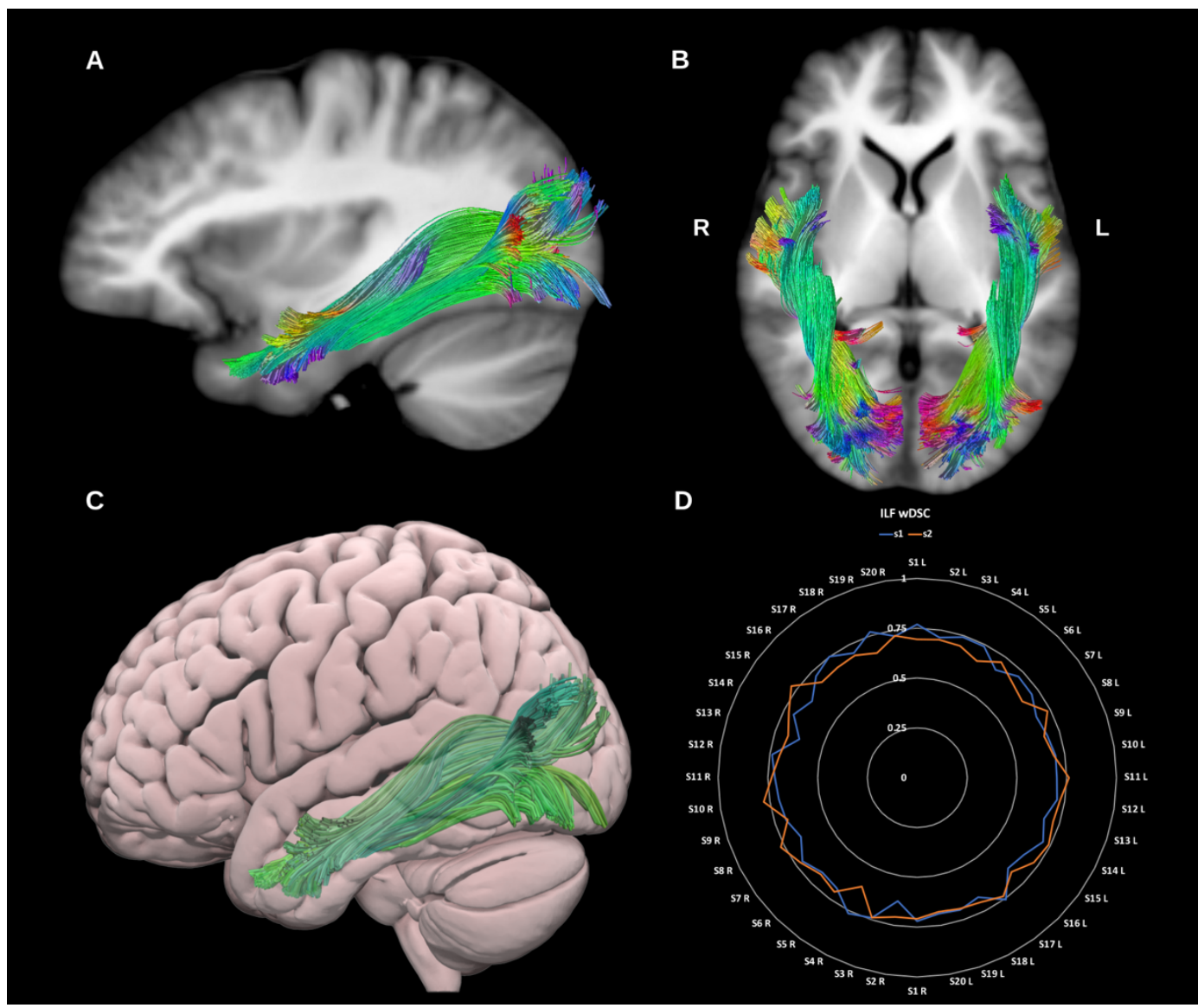

Figure 7: $(A)$ and $(B)$ show sagittal and axial slices of the T1-weighted group average image with the inferior longitudinal fasciculi (ILF) overlaid in directional color coding. (C) shows a lateral projection of the semitransparent MNI pial surface with the left ILF in green. (D) shows a radar plot of the wDSC scores (vertical range) of both ILFs as reconstructed using first session (blue) and second session (orange) data. Left sided bundles $(\mathrm{L})$ are on the first half of the circle and right ones $(\mathrm{R})$ are on the second half. $\mathrm{MNI}=$ Montreal Neurological Institute, $\mathrm{WDSC}=$ weighted dice similarity coefficient.

The ILF is a large association tract connecting the occipital and temporal lobes,

321 and may play an important functional role in visual memory and emotional processing.

322 It lies in direct contact with or close proximity to several bundles, including the UF,

323 IFOF, AF, optic radiations (ORs) and tapetal fibers of the corpus callosum (CC). Whilst

324 commonly depicted as a single bundle, the ILF may have up to four morphological

325 subdivisions which reflect its occipital termination points, and include lingual, cuneate, 
bioRxiv preprint doi: https://doi.org/10.1101/2021.10.13.464139; this version posted October 19, 2021. The copyright holder for this preprint (which was not certified by peer review) is the author/funder, who has granted bioRxiv a license to display the preprint in perpetuity. It is made available under aCC-BY 4.0 International license.

fusiform and dorso-lateral occipital subcomponents. However, given the lack of

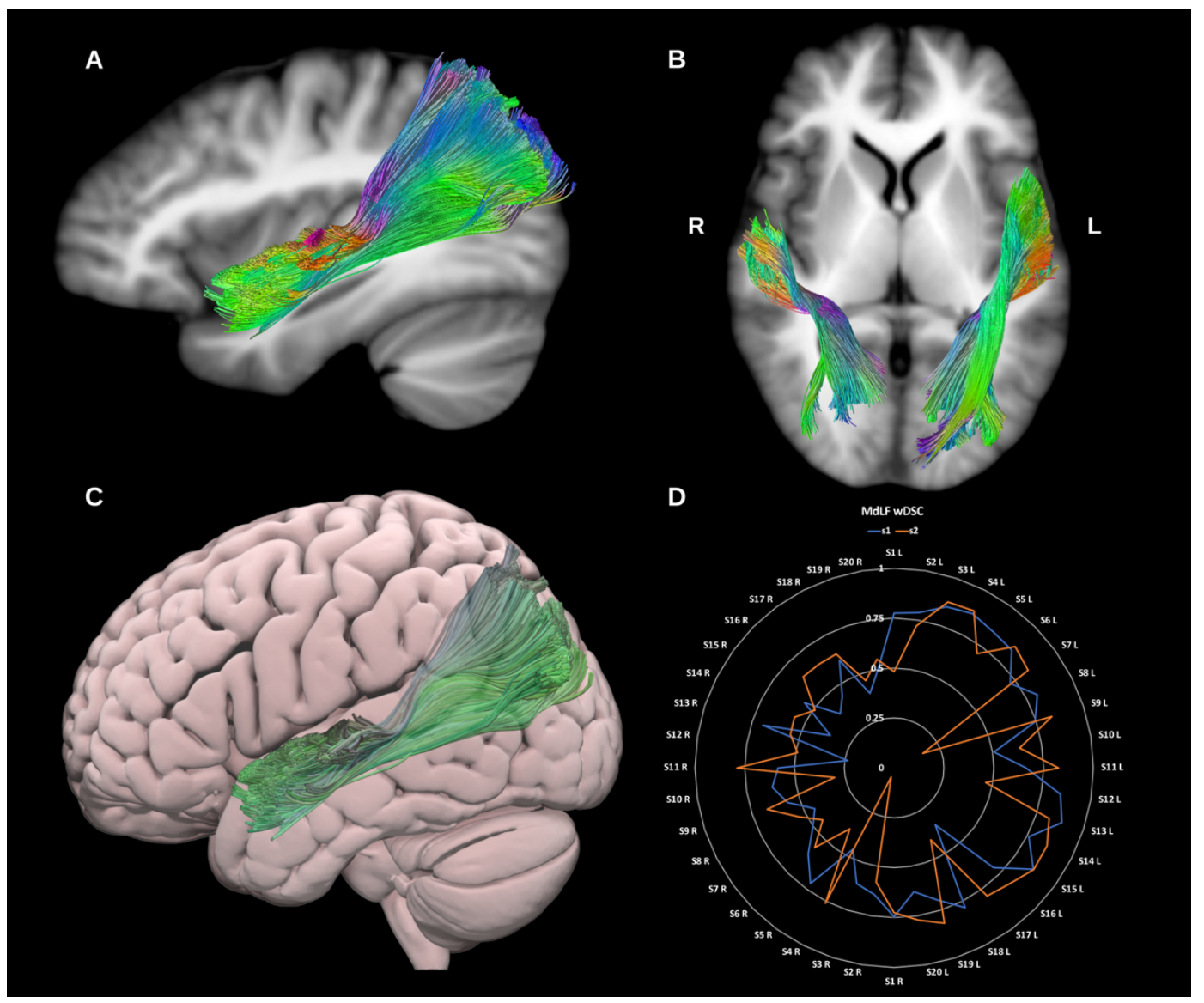

Figure 8: $(A)$, and $(B)$ show sagittal and axial slices of the T1-weighted group average image with the Middle longitudinal fasciculi (MdLF) overlaid in directional color coding. (C) shows a lateral projection of the semitransparent MNI pial surface with the left MdLF in green. (D) shows a radar plot of the wDSC scores (vertical range) of both MdLFs as reconstructed using first session (blue) and second session (orange) data. Left sided bundles $(\mathrm{L})$ are on the first half of the circle and right ones $(\mathrm{R})$ are on the second half. $\mathrm{MNI}=$ Montreal Neurological Institute, wDSC = weighted dice similarity coefficient.

The MdLF is a large association bundle which is hypothesized to play a role in 332 language, visual and auditory processing (De Witt Hamer et al., 2010; Dick and 333 Tremblay, 2012; Kalyvas et al., 2020). Broadly speaking it is thought to connect the 334 temporal pole, superior temporal gyrus, angular gyrus, superior parietal lobule and 335 precuneus forming a distinct association bundle that runs medial to the AF and lateral 336 to the IFOF (Makris et al., 2017, 2009; Maldonado et al., 2013; Menjot de Champfleur 
337 et al., 2013; Seltzer and Pandya, 1984; Wang et al., 2013), see figure 7. However,

338 there remains considerable debate about the anatomical description of the MdLF

339 owing to its relatively recent discovery in humans, and the evolution in its connectivity

340 profile as a result of differences in tracking algorithms. Indeed, an accurate

341 reconstruction of the MdLF is hindered by its close proximity to the extreme capsule,

342 ILF, IFOF and AF. The MdLF was generated for all datasets on both sides.

\subsubsection{Superior Longitudinal Fasciculus (SLF - I, II, and III)}

344 The SLF is a parieto-occipital association fiber system, located in the dorso-lateral 345 aspect of the cerebrum and generally believed to comprise three or four components 346 (de Schotten et al., 2011; Wang et al., 2016). Broadly speaking it connects the frontal 347 with the occipital, parietal and temporal lobes, see figure 9. The SLF is related to 348 language (Madhavan et al., 2014) visuo-spatial (Hong et al., 2019), and meta-cognitive 349 (Zheng et al., 2020) functions. When tracked as a whole the SLF is highly reproducible, 350 being successfully generated in all sessions for all datasets on both sides.

\subsection{SLF=|}

352 The SLF-I connects the superior parietal lobule and precuneus with posterior 353 superior frontal cortical areas. It is a rather short association bundle that is located above the level of the cingulate cortex (Wang et al., 2016). This was generated for all datasets on both sides but was the least reproducible of the SLF segments, most likely due to its small volume and low streamlines count.

\subsection{SLF-II}

SLF-II is located more infero-lateral to SLF-I and originates in the anterior intraparietal sulcus and the angular gyrus, terminating in the posterior regions of the superior and middle frontal gyri. SLF-II can be subdivided into two distinct subcomponents (Barbeau et al., 2020), the first is the dorsal component (SLF-IId), which connects the supramarginal and inferior parietal cortices to the dorsal middle frontal gyrus. The second is the ventral component (SLF-IIv), which is longer, more ventral and connects the supramarginal and inferior parietal cortices to the rostral middle frontal gyrus. The SLF-IId and SLF-Ilv were successfully reconstructed for all datasets on both sides. 
bioRxiv preprint doi: https://doi.org/10.1101/2021.1013.464139; this version posted October 19, 2021. The copyright holder for this preprint (which was not certified by peer review) is the author/funder, who has granted bioRxiv a license to display the preprint in perpetuity. It is made available under aCC-BY 4.0 International license.

\subsection{SLF-III}

SLF-III connects the intraparietal sulcus and inferior parietal lobule to the inferior

369 frontal gyrus (Barbeau et al., 2020). It is immediately superior and medial to the

370 arcuate fasciculus partially overlapping with the horizontal fibers of the Arcuate

371 fasciculus. The SLF-III was generated for all datasets on both sides.

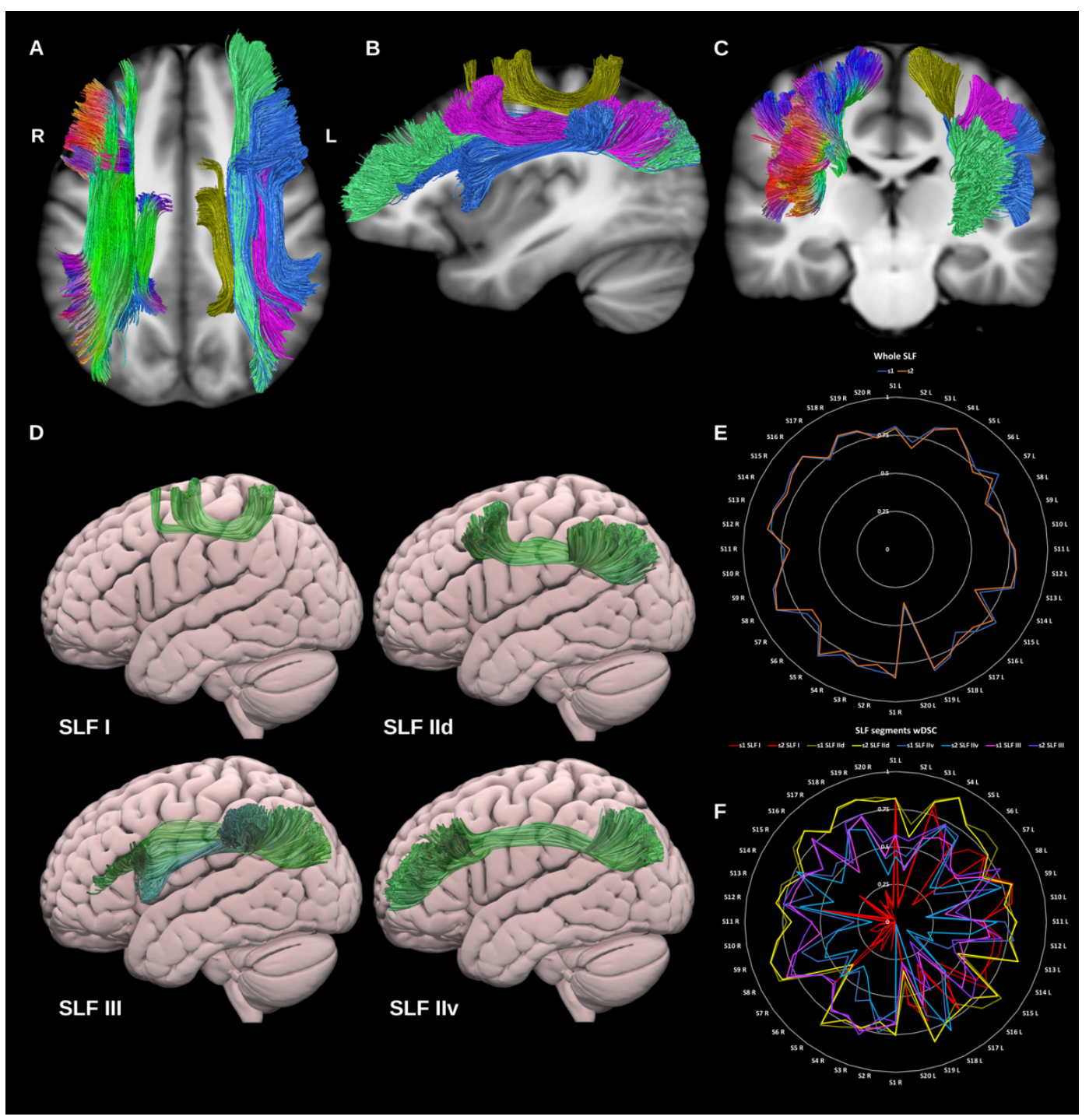

Figure 9: $(A),(B)$, and $(C)$ show sagittal and axial slices of the T1-weighted group average image with the superior longitudinal fasciculi (SLF) overlaid in directional color coding on the right side and show in gold (SLF-I), pink (SLF-IId), green (SLF-IIv) and blue (SLF-III). (D) shows a radar plot of the wDSC scores (vertical range) of both whole SLFs as reconstructed using first session (blue) and second session (orange) data. Left sided bundles $(L)$ are on the first half of the circle and right ones $(R)$ are on the second half. $(E)$ shows lateral projections of the semitransparent MNI pial surface with the left SLF I, IId, IIv, and III in green. (F) shows a radar plot of the wDSC scores (vertical range) of SLF components for each side. MNI= Montreal Neurological Institute, wDSC= weighted dice similarity coefficient. 


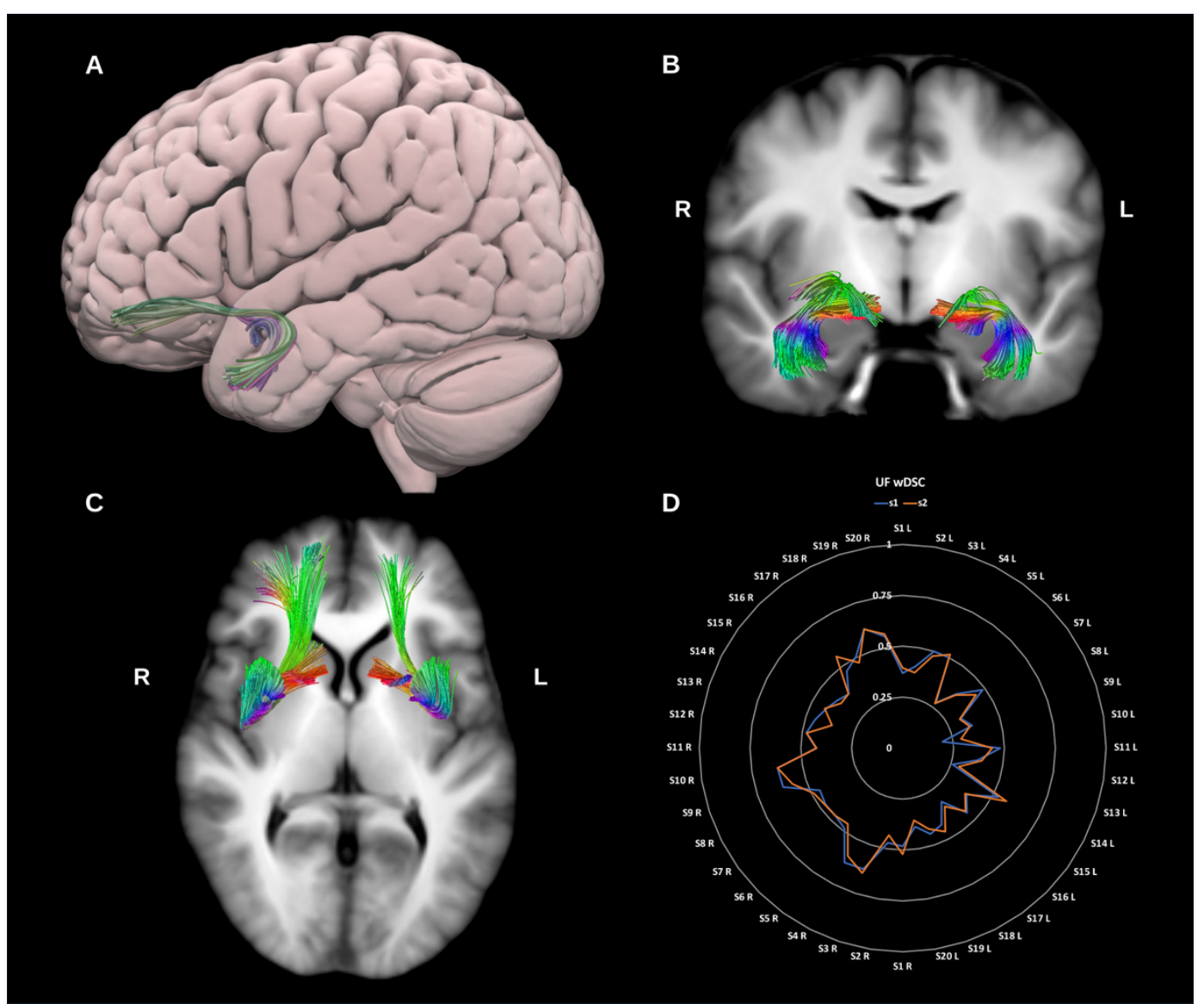

Figure 10: $(A)$ shows a lateral projection of the semitransparent MNI pial surface with the left uncinate fasciculus (UF) in directional color coding, (B) and (C) show coronal and axial slices of the T1-weighted group average image with the UF overlaid in directional color coding. (D) shows a radar plot of the wDSC scores (vertical range) of both UFs as reconstructed using first session (blue) and second session (orange) data. Left sided bundles (L) are on the first half of the circle and right ones $(\mathrm{R})$ are on the second half. $\mathrm{MNI}=$ Montreal Neurological Institute, $\mathrm{wDSC}=$ weighted dice similarity coefficient.

The UF is typically characterized as a hook shaped bidirectional association fiber

374 bundle linking the ventral, medial and orbital frontal lobes and rostral temporal lobes.

375 Whilst DTI typically reconstructs a short hook-shaped fasciculus (Kurki et al., 2013),

376 higher order dMRI reconstructions have revealed a more extensive fiber system which

377 expands into a fan shaped trajectory in the frontal lobe. Such higher order models

378 (e.g., CSD) in combination with microdissection suggest that the UF may be further 379 subdivided into 5 subcomponents (Hau et al., 2017). However, for the present 380 reconstruction, we opted to reconstruct the UF as one bundle for practicality, see figure

381 10. This was generated for all datasets on both sides. 


\subsubsection{Vertical Occipital Fasciculus (VOF)}

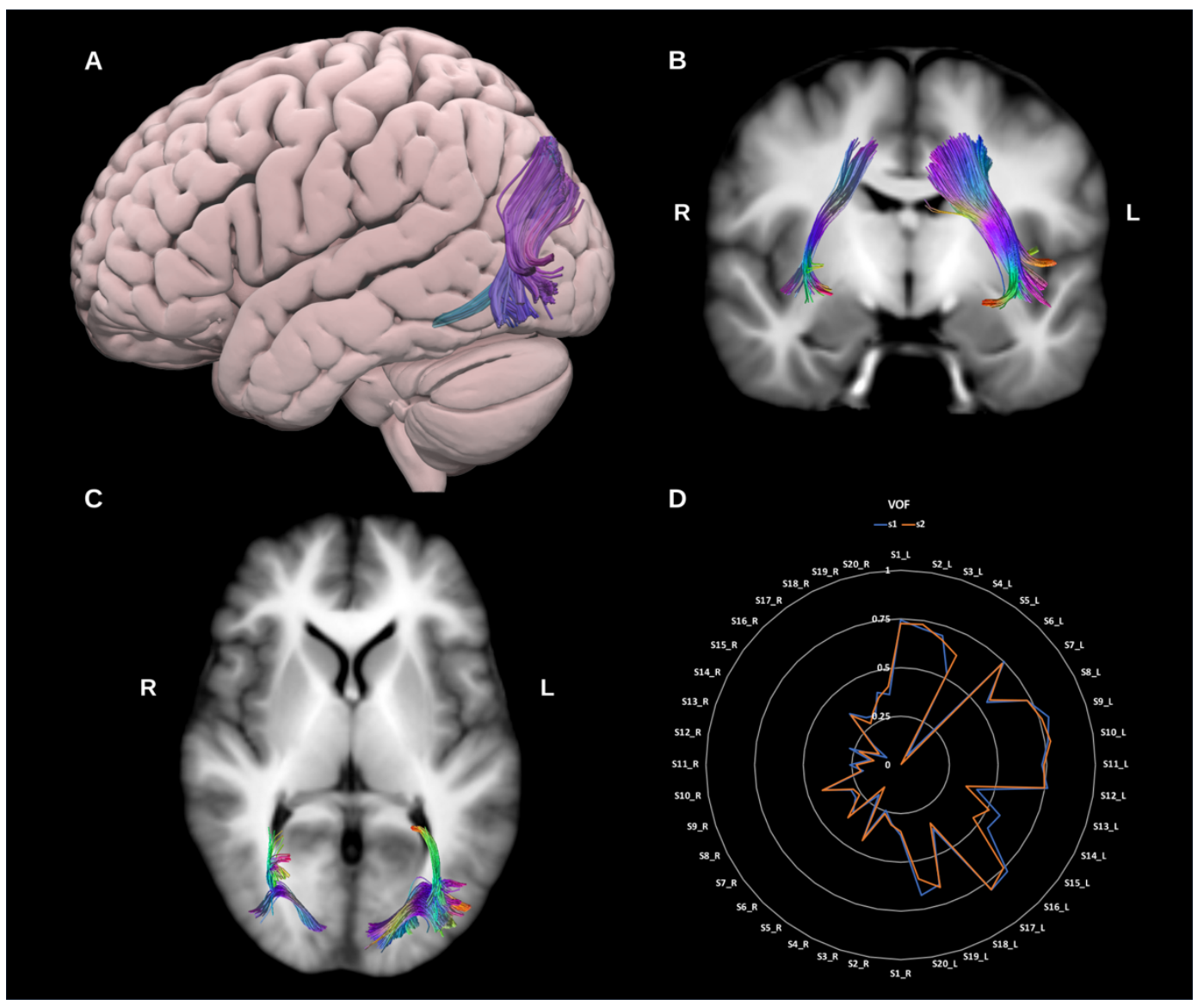

Figure 11: $(A)$ shows a lateral projection of the semitransparent MNI pial surface with the left vertical occipital fasciculus (VOF) in directional color coding, (B) and (C) show coronal and axial slices of the T1-weighted group average image with the VOF overlaid in directional color coding. (D) shows a radar plot of the wDSC scores (vertical range) of both VOFs as reconstructed using first session (blue) and second session (orange) data. Left sided bundles $(L)$ are on the first half of the circle and right ones (R) are on the second half. MNI = Montreal Neurological Institute, wDSC $=$ weighted dice similarity coefficient.

The VOF is described as a short slanted vertical association bundle in the lateral aspect of the occipital lobe. It connects the superior part of the occipital lobe and adjacent cortex of the occipito-parietal sulcus to the inferior aspect of the occipital lobe and adjacent occipito-temporal cortical areas (Jitsuishi et al., 2020; Schurr et al., 2019; Yeatman et al., 2014), see figure 11. This was generated for all datasets on both sides.

\subsubsection{Commissural fiber bundles (in alphabetical order)}

\subsubsection{1}

\section{Anterior Commissure (AC)}

The anterior commissure crosses the midline anterior to the pre-commissural columns of the fornix, above the basal forebrain and below the medial and ventral portion of the anterior limb of the internal capsule. It has two main parts, the first, 
bioRxiv preprint doi: https://doi.org/10.1101/2021.10.13.464139; this version posted October 19, 2021. The copyright holder for this preprint (which was not certified by peer review) is the author/funder, who has granted bioRxiv a license to display the preprint in perpetuity. It is made available under aCC-BY 4.0 International license.

anterior division which includes the olfactory decussation and the second, largest

394 division which connects the temporal lobes, occipital lobes, and predominantly the bilateral amygdalae (van Meer et al., 2016). During its course the AC intersects with the UF, ILF, sagittal stratum and optic radiations, which complicates tracking the true extent of the structure (Çavdar et al., 2021; Choi et al., 2011; Kikinis et al., 2015; Peltier et al., 2011; van Meer et al., 2016; Wilde et al., 2006), see figure 12. The AC was reconstructed successfully in both sessions for all but one subject.

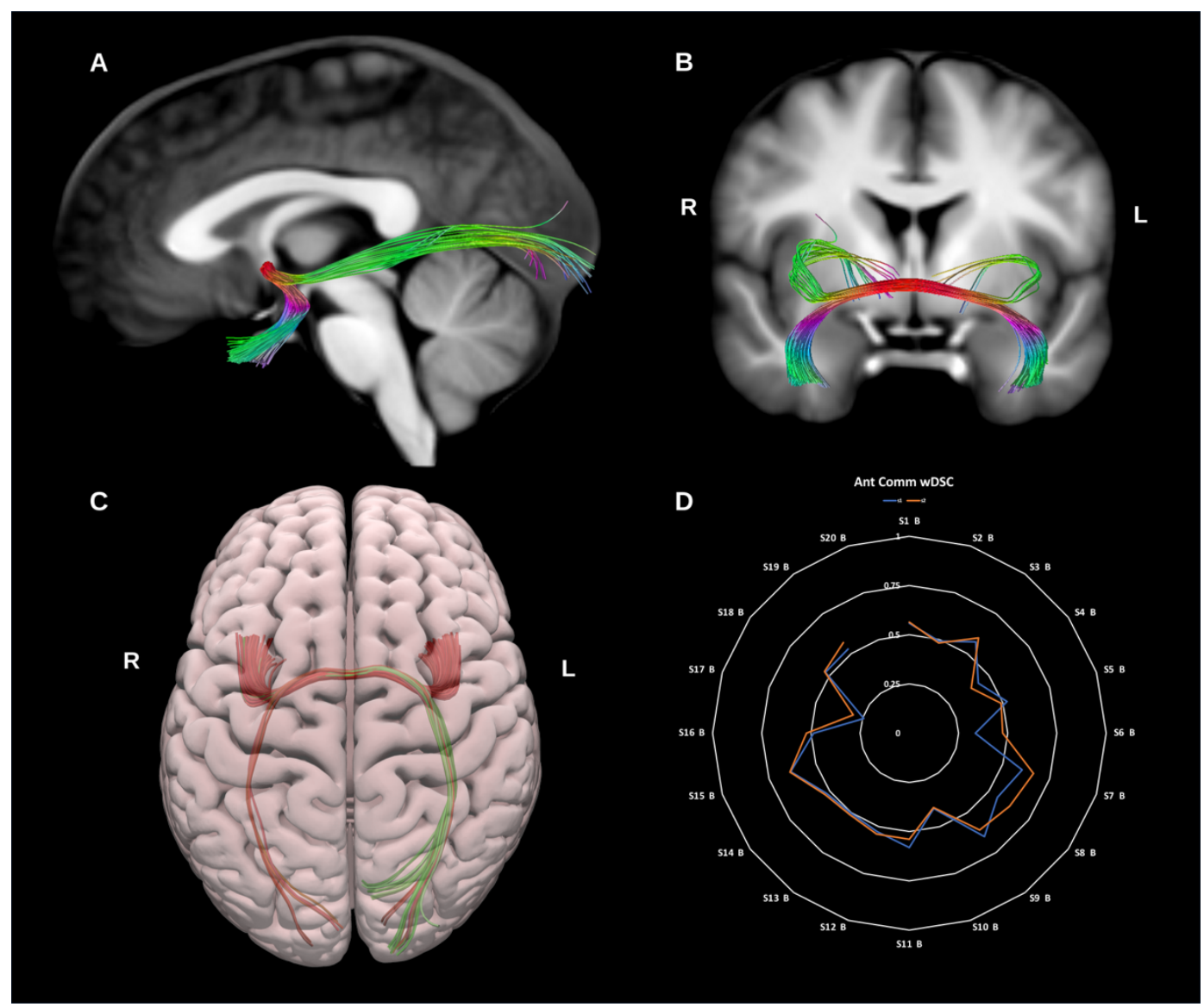

Figure 12: $(A)$ and $(B)$ show sagittal and coronal slices of the T1-weighted group average image with the anterior commissure (Ant. Comm.) overlaid in directional color coding, (C) shows a superior projection of the semitransparent MNI pial surface with the Ant. Comm. in directional color coding, and (D) shows a radar plot of the wDSC scores (vertical range) of the Ant. Comm. as reconstructed using first session (blue) and second session (orange) data. MNI = Montreal Neuro- 
bioRxiv preprint doi: https://doi.org/10.1101/2021.10.13.464139; this version posted October 19, 2021. The copyright holder for this preprint (which was not certified by peer review) is the author/funder, who has granted bioRxiv a license to display the preprint in perpetuity. It is made available under aCC-BY 4.0 International license.

401

402

403

404

405

406

407

408

409

\subsubsection{Corpus Callosum (CC)}

The corpus callosum is the largest fiber bundle in the human brain and connects the right and left cerebral hemispheres, and hence its primary function is interhemispheric information transfer and integration. Traditionally the CC is divided into several subcomponents: the rostrum, genu, body, isthmus, splenium and tapetum. It is common in DTI based literature to find three subdivisions: the genu, which forms the forceps minor and connects left and right prefrontal and anterior cingulate cortices; the callosal body, and the splenium, which forms the forceps major and connects left and right posterior parietal, medial occipital and medial temporal cortices.

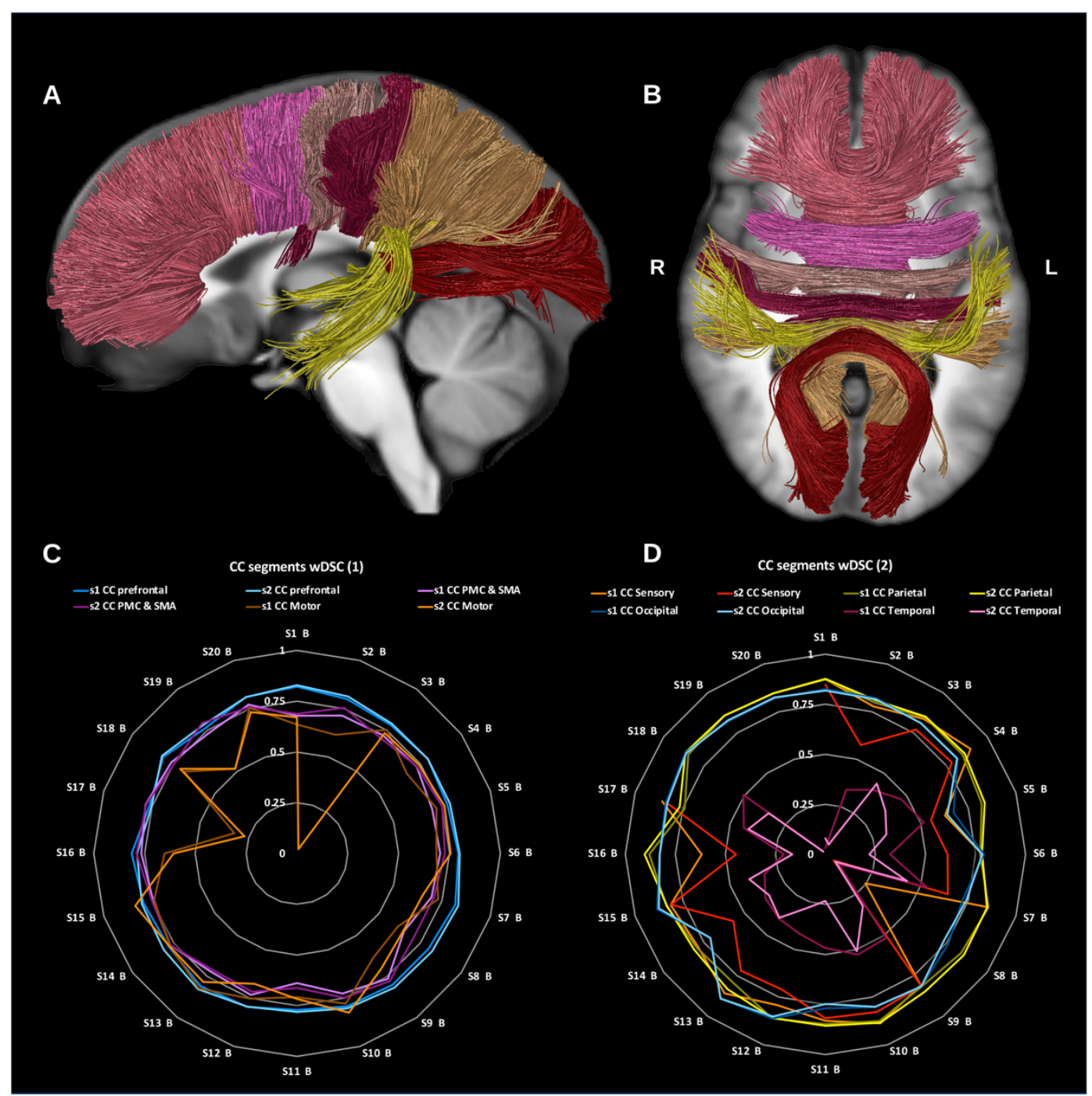

Figure 13: $(A)$ and $(B)$ show sagittal and axial slices of the T1-weighted group average image with the all segments of corpus callosum (CC). Radar plots of wDSC (vertical ranges) resulting from comparison to HCP-template bundles, (C) Prefrontal CC (blue and light blue), premotor and supplementary motor CC (pink and fuschia), motor CC (dark and light orange), (D) sensory CC (orange and red), parietal CC (gold and yellow), occipital CC (blue and light blue), and temporal CC (fuschia and pink). $\mathrm{PMC}=$ premotor cortex, $\mathrm{SMA}=$ supplementary motor cortex . 
410 Improvements in fiber-tracking algorithms and integration with functional data have led

411 to alternative parcellation strategies (Catani, 2006; Fabri et al., 2014; Nazem-Zadeh

412 et al., 2012; Ohoshi et al., 2019, 2019; Phillips et al., 2013). Here we have

413 reconstructed the $\mathrm{CC}$ into 7 segments comprising the prefrontal, premotor, motor,

414 sensory, parietal, occipital and temporal callosal fibers, see figures 13 and 14 .

\section{3.1.2.2.1 Prefrontal corpus callosum (CC PreF):}

416 This was reconstructed for all datasets using the bilateral prefrontal cortices and

417 the genu and anterior third of the body of the corpus callosum.

420 This was reconstructed for all datasets using the caudal middle frontal gyri, the supplementary motor areas bilaterally and the central body of the corpus callosum.

\section{$422 \quad 3.1 .2 .2 .3 \quad$ Motor corpus callosum (CC motor):}

423 This segment connects the primary motor cortices (precentral gyri) of both

424 hemispheres via the posterior third of the body of the corpus callosum. The motor CC 425 was generated for all datasets.

\section{3.1.2.2.4 Sensory corpus callosum (CC sensory):}

427 This was reconstructed using the primary sensory cortices (postcentral gyri) on 428 both sides via the posterior third of the body and splenium of the corpus callosum in 429 the midline. The sensory CC was reconstructed for all except 4 datasets.

\section{$430 \quad 3.1 .2 .2 .5 \quad$ Parietal corpus callosum (CC parietal):}

431 This was reconstructed for all data using the whole parietal lobes bilaterally and 432 the splenium of the corpus callosum.

\section{3.1.2.2.6 Occipital corpus callosum (CC occipital):}

434 This was reconstructed for all data using the occipital lobes on both sides and the 435 splenium of the corpus callosum. 
bioRxiv preprint doi: https://doi. org/10.1101/2021 10.13.464139. this version posted October 19, 2021. The copyright holder for this preprint (which was not certified by peer review) is the author/funder, who has granted bioRxiv a license to display the preprint in perpetuity. It is made available under aCC-BY 4.0 International license.

This was reconstructed using the lateral aspect of the temporal lobes bilaterally and the splenium of the corpus callosum in the midline. The temporal $\mathrm{CC}$

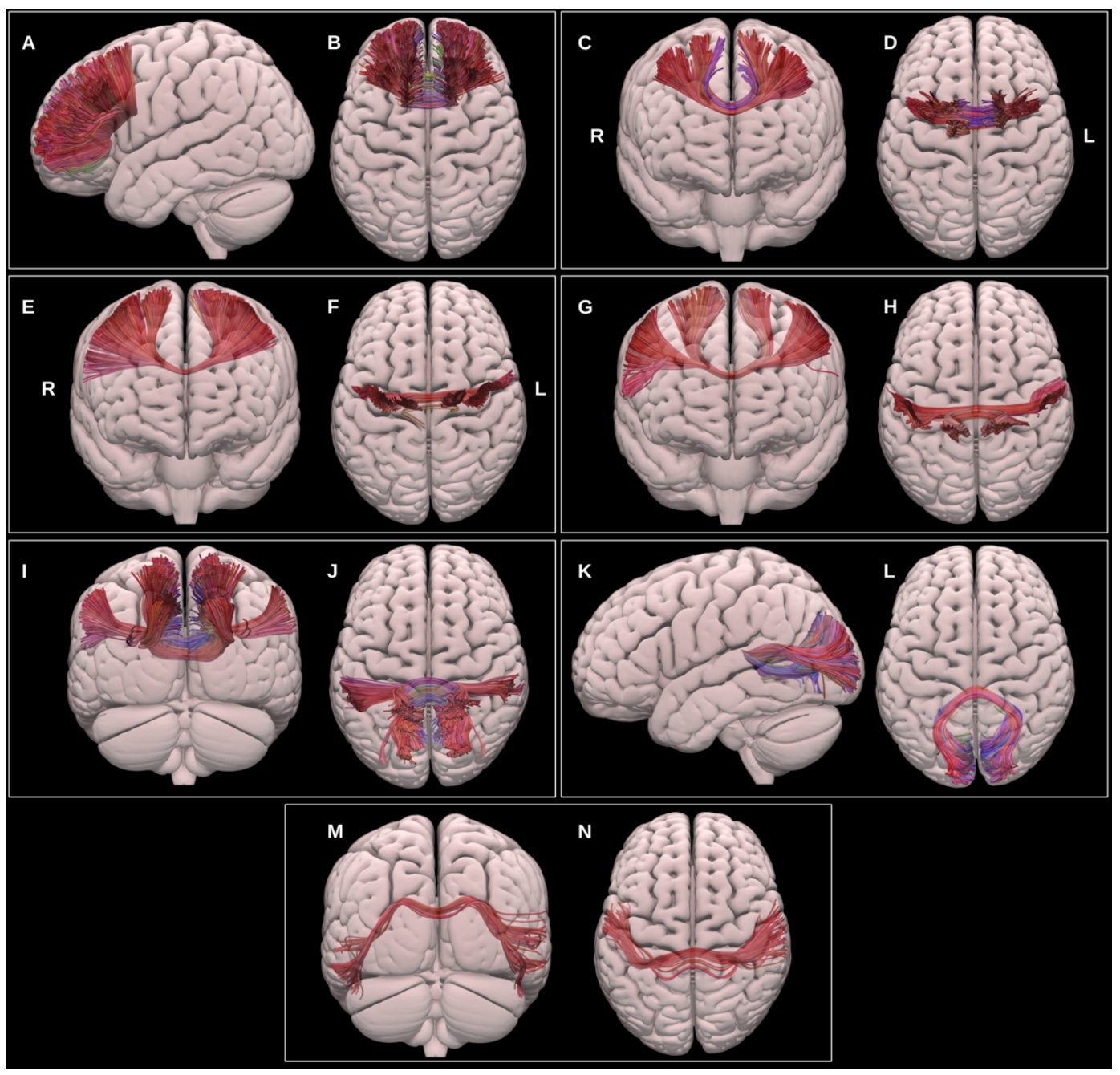

Figure 14: Multiple projections of the semitransparent MNI pial surface, with lateral and superior views showing the prefrontal $C C(A \& B)$, anterior and superior views showing the PMC and SMA $C C(C \& D)$, anterior and superior views showing the motor $C C(E \& F)$, anterior and superior views showing the sensory $C C(G \& H)$, posterior and superior views showing the parietal $C C$ (I \& J), lateral and superior views showing the occipital CC, and posterior and superior views showing the temporal CC (M \& N).

\subsubsection{Projection fiber bundles (in alphabetical order)}

442 The primary dorsal ascending tracts originate in the dorsal columns of the spinal 443 cord, ascend through the brain stem medulla as the medial lemniscus to the thalamic 444 ventral-posterior medial (VPM) and lateral (VPL) nuclei and via the internal capsule to 
bioRxiv preprint doi: https://doi. org/10.1101/2021.10.13.464139. this version posted October 19, 2021. The copyright holder for this preprint (which was not certified by peer review) is the author/funder, who has granted bioRxiv a license to display the preprint in perpetuity. It is made available under aCC-BY 4.0 International license.

445 the primary somatosensory cortex in the postcentral gyrus (Jang and Seo, 2015; Peng 446 et al., 2019a), see figure 15. The ML was reconstructed using the UKBB derived 447 posterior brainstem VOIs, the VPM and VPL thalamic nuclei, and primary sensory 448 cortices. This was generated in all datasets on both sides.

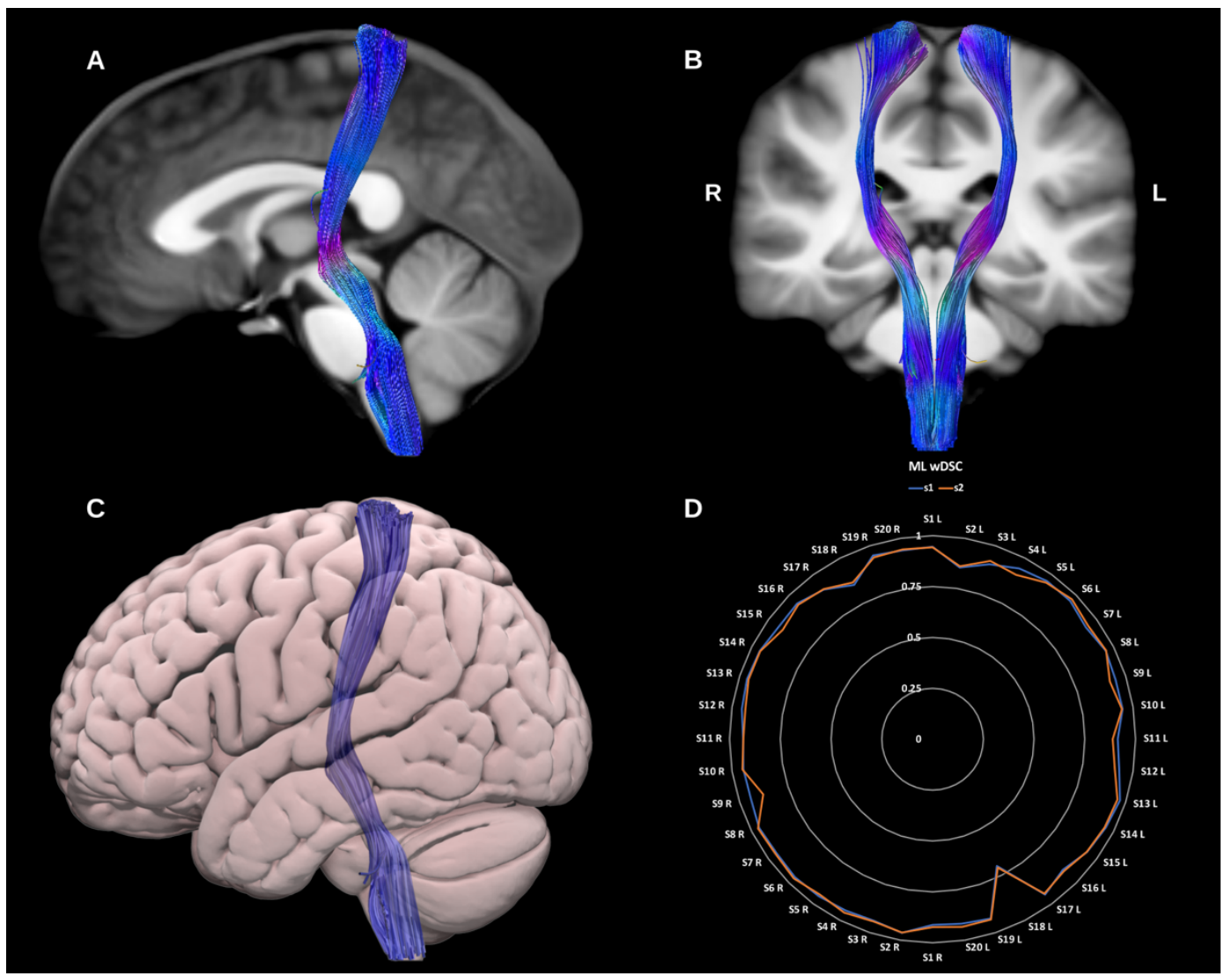

Figure 15: $(A)$ and $(B)$ show sagittal and coronal slices of the T1-weighted group average image with the medial lemniscus (ML) overlaid in directional color coding, (C) shows a lateral projection of the semitransparent MNI pial surface with the ML blue, and (D) shows a radar plot of the WDSC scores (vertical range) of the ML as reconstructed using first session (blue) and second session (orange) data. Left sided bundles $(L)$ are on the first half of the circle and right ones $(R)$ are on the second half. $\mathrm{MNI}=$ Montreal Neurological Institute, $\mathrm{wDSC}=$ weighted dice similarity coefficient. 


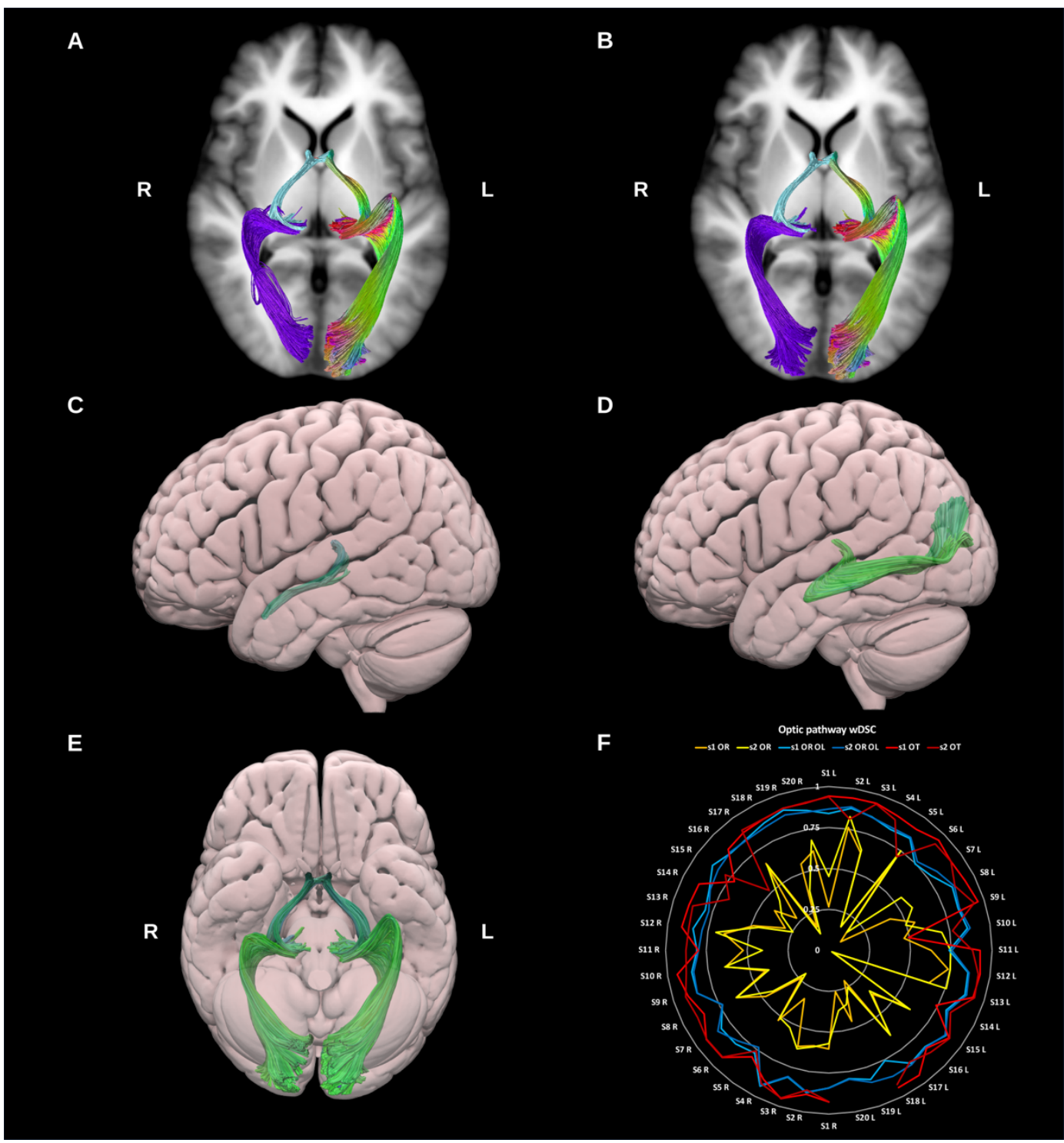

Figure 16: $(A)$ and $(B)$ show axial slices of the T1-weighted group average image with the overlaid optic tracts (light blue) and (A) classic optic radiations (ORs) (purple), and (B) whole occipital lobe optic radiations (OR OL) (purple) on the right side and directional color coding on the left side. (C) \& (D) show lateral projections of the semitransparent MNI pial surface with the optic tract (OT) (C) and optic radiation $(D)$. E shows an inferior projection of the surface view for the entire optic pathway on both sides. (F) shows a radar plot of the wDSC scores (vertical range) of the optic tracts and both versions of the optic radiations. Left sided bundles $(L)$ are on the first half of the circle and right ones $(\mathrm{R})$ are on the second half. MNI = Montreal Neurological Institute, wDSC $=$ weighted dice similarity coefficient.

The ORs connect the lateral geniculate nucleus of the thalamus to the primary

452 visual cortex within the occipital lobe. They are critical for visual processing and

453 present important challenges during surgical procedures involving the temporal lobe.

454 Notably, Meyer's loop presents a particular challenge for tractography (Mehra and

455 Moshirfar, 2021). This portion of the OR, which exhibits significant inter-individual 
456 variability, is characterized by a sharp anterior projection in the anterior temporal lobe

457 that then bends posteriorly to join the sagittal stratum. Moreover, within the anterior 458 loop, OR fibers may intersect with fibers of the anterior commissure, ILF and tapetum,

459 increasing the likelihood of spurious streamlines (Chamberland et al., 2018; Goga and

460 Türe, 2015; Lim et al., 2015; Martínez-Heras et al., 2015), see figure 16. We generated

461 two representations of the optic radiations, first using a classical anatomical definition

462 with only the pericalcarine cortex as a cortical inclusion VOI, and second using the 463 entire occipital lobe as a cortical inclusion VOI. Both versions of the ORs were 464 successfully reconstructed for all datasets on both sides.

\section{$465 \quad 3.1 .3 .3 \quad$ Optic Tract (OT)}

466 The OT is formed of decussating axons from the contra-lateral optic nerve as well 467 as non-decussating fibers from the ipsilateral optic nerve. It extends from the optic 468 chiasm to the lateral geniculate nucleus of the thalamus (Hofer et al., 2010; Mehra and 469 Moshirfar, 2021; Wu et al., 2012), see figure 16. The OTs reconstruction failed in 3 470 datasets on the left side only.

\section{3.1.3.4 Pyramidal Tract (PyT)}

472 The pyramidal tract fibers carry motor impulses from the cerebral cortex to the 473 spinal cord through the brainstem. Here we reconstruct the Pyramidal tract (PyT) as a 474 whole including the primary motor and sensory cortices along with the premotor, 475 supplementary motor and parietal proprioceptive cortices superiorly and the whole 476 brain stem inferiorly. We also provide dissections of this bundle based on specific 477 subsystems of the sensory-motor network (Chenot et al., 2019; Jeong et al., 2013; 478 Peng et al., 2019b; Zhang et al., 2010), see figure 17. The whole PyT was successfully 479 reconstructed for all datasets on both sides, all its subcomponents were reconstructed 480 successfully except for the premotor component, which failed in 6 datasets and was 481 found to be the least reproducible. 


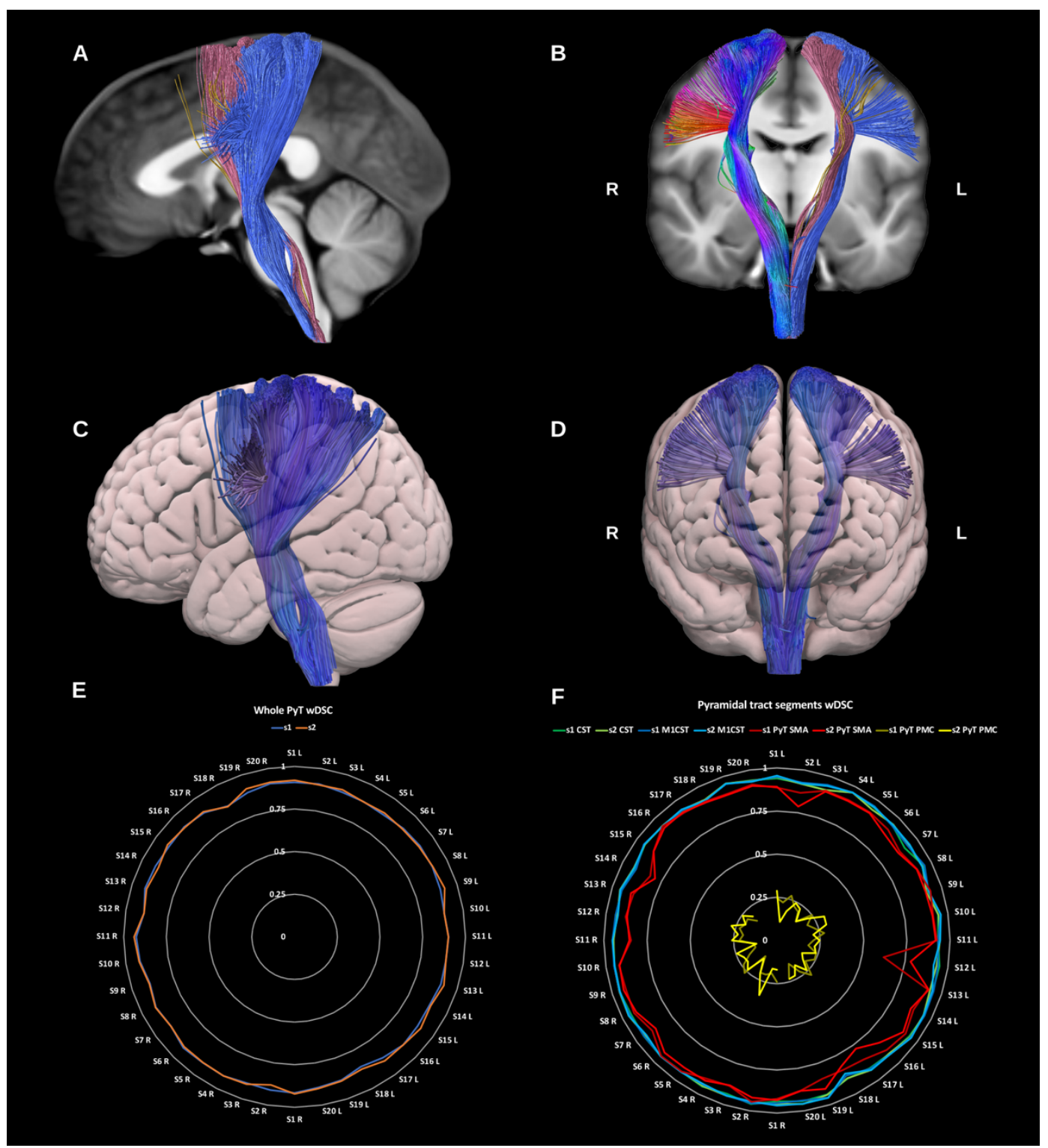

Figure 17: $(A)$ and $(B)$ show sagittal and coronal slices of the T1-weighted group average image with the whole pyramidal tract (PyT_all) overlaid in directional color coding on the right side and its different components on the left side in solid colors, premotor pyramidal tract (PyT_PMC) is shown in yellow, the supplementary motor pyramidal tract (PyT_SMA) in pink and the corticospinal tract (CST) in blue, (C) and (D) show lateral and anterior projections of the semitransparent $\mathrm{MNI}$ pial surface with the whole PyT on both sides shown in blue. Radar plots of wDSC (vertical ranges) resulting from comparison to HCP-template bundles are shown in $(E)$ for the whole pyT, and in (F) for the different pyramidal tract segments. Left sided bundles $(L)$ are on the first half of the circle and right ones $(\mathrm{R})$ are on the second half. $\mathrm{MNI}=$ Montreal Neurological Institute, $\mathrm{wDSC}=$ weighted dice similarity coefficient, $\mathrm{PyT}=$ pyramidal tract, $\mathrm{CST}=$ corticospinal tract, $\mathrm{M} 1 \mathrm{CST}=$ motor only $\mathrm{CST}, \mathrm{SMA}=$ supplementary motor area, $\mathrm{PMC}=$ premotor cortex, $\mathrm{S}=$ session, $\mathrm{S}=$ subject, $\mathrm{L}=$ left, 
The CST descends predominantly from the primary motor areas of the precentral

gyrus and somatosensory areas of the postcentral gyrus through the corona radiata, posterior half of the posterior limb of the internal capsule, and cerebral peduncles to the rostral brainstem where it forms the medullary pyramids before crossing the reconstructions of this bundle, one using the primary motor and sensory cortices (CST) and another using only the primary motor cortices, excluding the postcentral gyri 492 (M1_CST).

\subsection{Premotor pyramidal Tract (PyT PMC)}

494 This represents the pyramidal tract fibers originating in the dorsal premotor cortex 495 (caudal middle frontal gyrus) and descending to the brainstem via the anterior half of 496 the posterior limb of the internal capsule. This bundle had the lowest number of 497 streamlines and was the least reproducible of the pyramidal tract components.

\subsection{Supplementary motor pyramidal Tract (PyT SMA)}

499 This represents the pyramidal tract fibers originating in the supplementary motor 500 area and descending to the brainstem via the centrum semiovale and the anterior half 501 of the posterior limb of the internal capsule.

503 The thalamic radiations are a group of 4-5 thalamo-cortical projection bundles 504 comprising both afferent and efferent fibers. Collectively, the fibers of the thalamic 505 radiations can be described as fanning out towards the cortex and banding up as they 506 approach the thalami. As they run through the internal capsule and corona radiata they 507 intersect with several other projection bundles, which in addition to the complex nature 508 of thalamic microanatomy, can complicate reconstruction (Behrens et al., 2003; kakou 509 et al., 2017; Lyness et al., 2014; Tsao et al., 2015; Younes et al., 2019), see figure 18. 510 The posterior thalamic radiation is represented by the extended version of the optic 511 radiation (OR OL) including the entire occipital cortex. All thalamic radiations were 512 reconstructed using the whole thalamus as an inclusion VOI and excluding non513 contributing thalamic nuclei, e.g., the VPM and VPL nuclei were excluded for the 514 anterior thalamic radiation. 


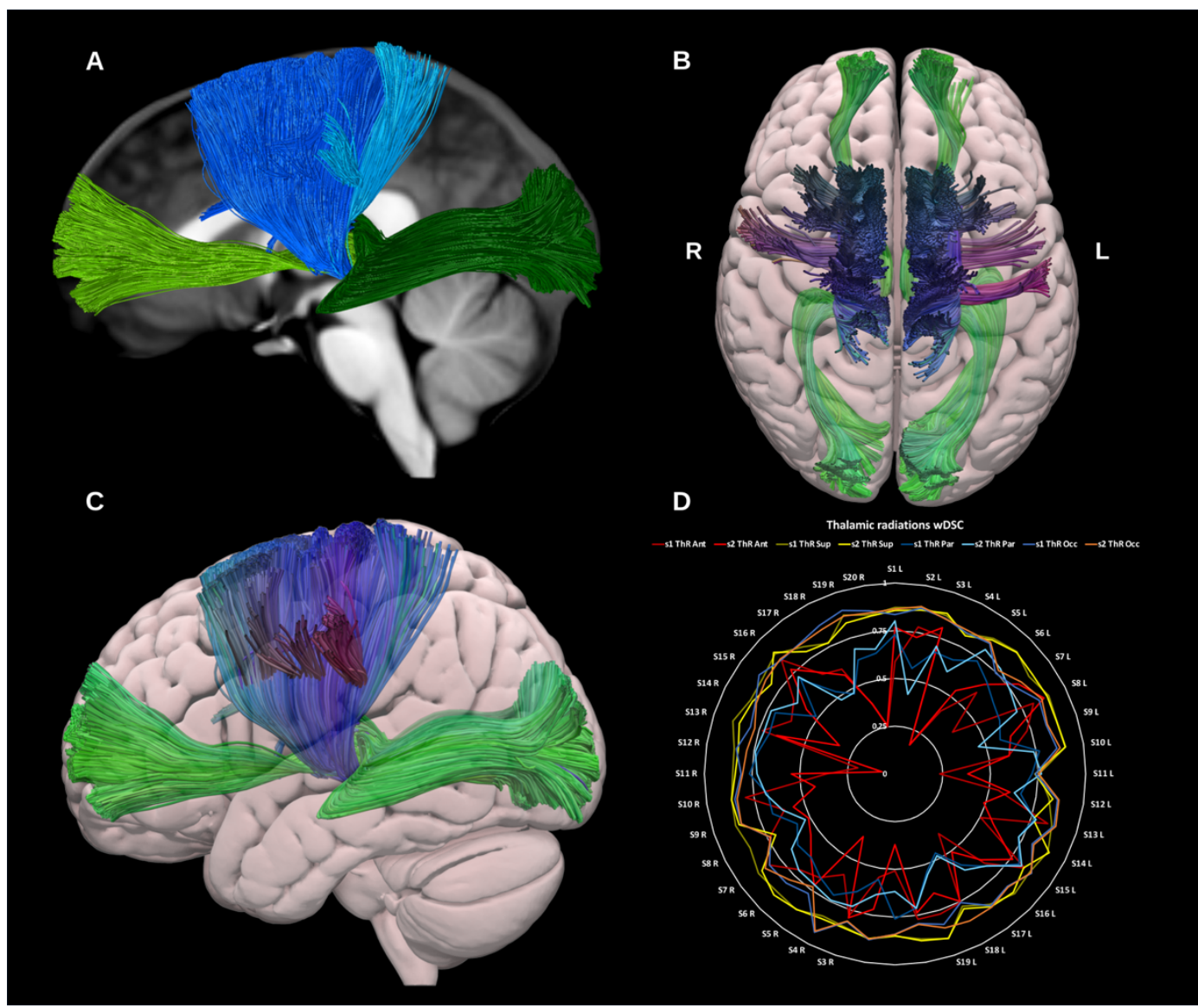

Figure 18: $(A)$ shows a sagittal view of the T1-weighted group average image with the thalamic radiations overlaid in different colors. The anterior thalamic radiation is shown in light green, the superior thalamic radiation in blue, the parietal thalamic radiation in turquoise, and the posterior thalamic radiation in dark green. $(B)$ and $(C)$ show superior and lateral projections of the semitransparent MNI pial surface with the anterior and posterior thalamic radiations in green, and the superior and sensory thalamic radiations in blue. (D) shows a radar plot of the wDSC scores (vertical range) of the different thalamic radiations. Left sided bundles $(L)$ are on the first half of the circle and right ones $(\mathrm{R})$ are on the second half. MNI = Montreal Neurological Institute, $\mathrm{wDSC}=$ weighted dice similarity coefficient, ThR Ant $=$ anterior thalamic radiation, ThR Sup $=$ superior thalamic radiation, ThR Par $=$ parietal thalamic radiation, $\mathrm{ThR}$ Occ $=$ occipital thalamic radiation $=$ Optic radiation to whole occipital lobe.

\subsection{Anterior thalamic radiation (ATR)}

516 The ATR connects the dorso-medial (DM), dorso-lateral (DL) and anterior thalamic 517 (ATN) nuclei to the prefrontal cortex and is thought to be involved in executive 518 functions and complex planning (Niida et al., 2018). The ATR was reconstructed using 519 the thalamus excluding all nuclei but the DM, DL, and ATN, and the ipsilateral medial 520 and rostral prefrontal cortices. The ATR was reconstructed for all datasets on both 521 sides. 


\subsection{Superior thalamic radiation (STR)}

523

524

525

526

The STR connects the ventral thalamic nuclei to the motor and sensory cortices via the superior thalamic peduncle, posterior limb of internal capsule, and the corona radiata, conducting cerebellar and basal ganglia input to the motor cortex (BoschBouju et al., 2013). The STR was reconstructed using the thalamus, excluding the dorso-medial, dorso-lateral and pulvinar nuclei, and the ipsilateral primary motor, supplementary motor and dorsal premotor cortices (Younes et al., 2019). The STR was successfully reconstructed for all datasets on both sides.

\subsection{Parietal thalamic radiation (PaTR)}

This was reconstructed using the entire parietal lobe as a cortical include and the thalamus excluding all except the VPM, and VPL nuclei. This resulted in a bundle of streamlines connecting the thalamic VPM and VPL predominantly to the primary sensory cortex. The PaTR was successfully reconstructed in all datasets on both sides.

\subsection{Posterior thalamic radiation (PoTR)}

This bundle was considered to be the same as the extended optic radiation using the entire occipital lobe, and was not reconstructed separately but will be added in future versions.

\subsubsection{Cerebellar bundles (in alphabetical order)}

\subsubsection{Dentato-rubro-thalamic tract (DRTT)}

The DRTT is the main efferent pathway from the cerebellum to the cerebral cortex and an important subdivision of the SCP in the context of deep brain stimulation-based neurosurgery. It originates in the dentate nucleus of the cerebellum, ascends through the brain stem where the majority of its constituent axons cross the midline at the superior cerebellar decussation (SCP) to synapse with the contra-lateral red nucleus (RN) in the midbrain (Coenen et al., 2020; Kwon et al., 2011; Mollink et al., 2016; Nowacki et al., 2018), see figure 19. From here the DRTT continues to the ventrolateral $(\mathrm{VL})$ and ventromedial (VM) nuclei of the thalamus. Technically the DRTT terminates in the thalamus; however, for functional completeness we extend its trajectory to terminate in the primary motor cortex. 
Whilst an ipsilateral component of the DRTT has been described in the literature,

553 for the current purpose we have limited the reconstruction to the classical/contralateral

554 DRTT. The left DRTT reconstruction failed in 4 datasets, while the right failed in 9

555 datasets. The overall DRTT wDSC showed a median of 0.498 , minimum of 0.078 and

556 Max.A.I.D. of 0.203. Additionally, we generated template DRTT bundles with 2,000

557 and 50,000 streamlines to illustrate the influence of the number streamline seeding

558 attempts on tracking outcome and subsequent template-based reproducibility

559 (supplementary figure 1).

$560 \quad 3.1 .4 .2 \quad$ Inferior cerebellar peduncle (ICP)

561 The ICP consists mainly of afferent sensory fibers projecting from the spinal cord

562 to the cerebellum. The ICPs are functionally involved in the maintenance of balance

563 and posture via the integration of proprioceptive sensory and motor functions (Voogd,

564 2004). Microdissection studies reveal that it consists of four afferent bundles and 1

565 efferent bundle (Lingford-Hughes and Kalk, 2012), however here we reconstruct it as

566 a single lateralized bundle using the dentate, fastigial and interposed nuclei of the

567 cerebellum, and the ipsilateral medulla oblongata (Salamon et al., 2007; van Baarsen

568 et al., 2016), see figure 19. The ICP was generated for all datasets on both sides.

\section{3.1.4.3 Middlle cerebellar peduncle (MCP)}

570 The MCPs are large paired bundles that connect the brainstem to the cerebellum

571 on both sides. They are often reconstructed in neuroimaging studies as a single

572 commissural bundle connecting both cerebellar hemispheres (Leitner et al., 2015).

573 The MCPs are thought to play a role in the modulation of skilled manual motor

574 functions (Lingford-Hughes and Kalk, 2012) and have been shown to consist of three

575 sub-fascicles (superior, inferior and deep) by microdissection studies (Jhaveri et al.,

576 2018). We reconstructed the MCP as a whole for each side using the contra-lateral

577 pons and cerebellar cortex. The resulting streamlines cross the midline in the pons, in

578 line with its neuroanatomical definition. (Re et al., 2017; van Baarsen et al., 2016;

579 Voogd, 2004), see figure 19. The MCP was generated for all datasets on both sides. 


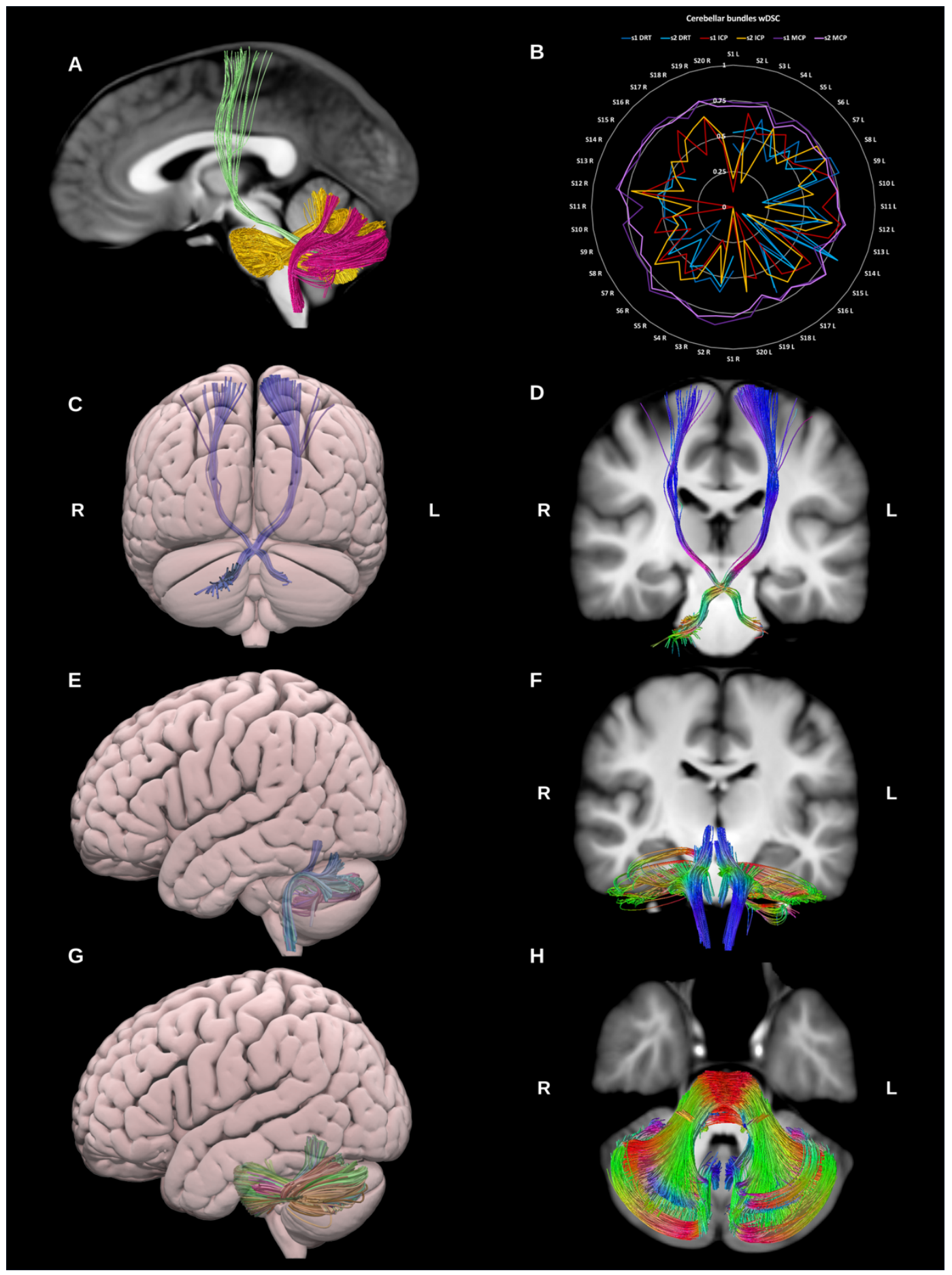

Figure 19: (A) shows a sagittal image of the T1-weighted group average image with the three cerebellar bundles shown in green for the dentato-rubro-thalamic tract (DRTT), inferior cerebellar peduncle (ICP) in fuschia, and in gold the middle cerebellar peduncle (MCP). (B) shows radar plots of WDSC (vertical range) for these three bundles per session. Left sided bundles $(\mathrm{L})$ are on the first half of the circle and right ones $(R)$ are on the second half. (C) and (D) shows a posterior projection surface view and a coronal slice with the DRTT overlaid. (E) and (F) show the ICPs shown on a lateral projection surface view, and a coronal slice respectively. $(G)$ and $(H)$ show the MCP in lateral projection surface view, and axial slice T1. wDSC = weighted dice similarity coefficient, $\mathrm{MNI}=$ Montreal Neurological Institute, missing results indicate failed tractography. 


\subsection{HCP test-retest data results}

In total we successfully reconstructed 2676 out of the 2720 attempted bundle reconstructions. The following bundles could not be reconstructed in all datasets: the

584 DRTT (13 failures), premotor pyramidal tract (6 failures), optic tract (3 failures), fornix 585 (15 failures), anterior commissure (2 failures), sensory CC (4 failures), and temporal 586 CC (1 failure). Results of inter-session pair-wise similarity analysis per bundle for the

587 different HCP test-retest subjects are shown as violin plots in figure 20.

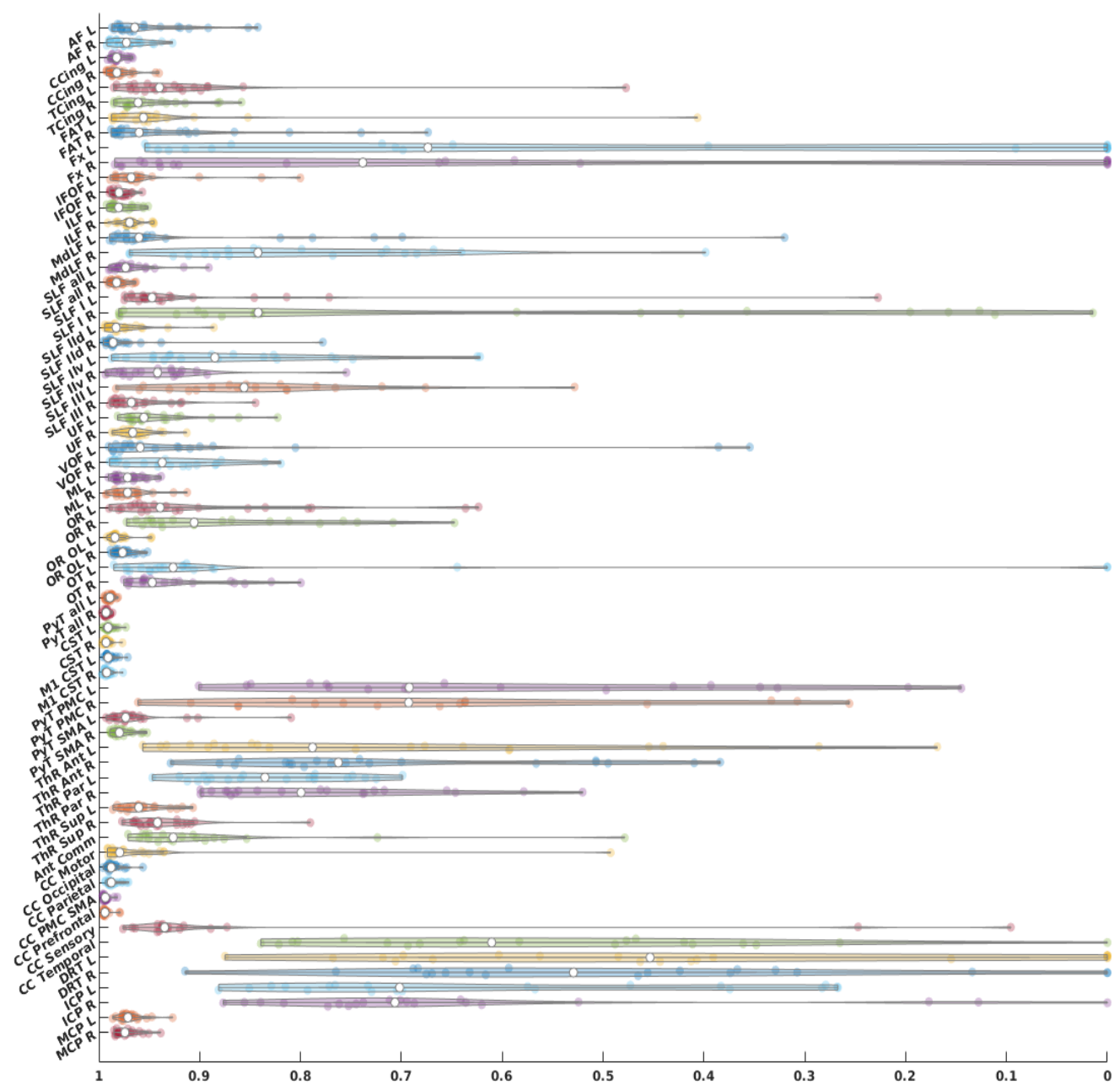

Figure 20: HCP tractograms wDSC scores depicted as violin plots. $\mathrm{wDSC}=$ weighted dice score, $\mathrm{AF}=$ arcuate fasciculus, $\mathrm{CCing}=$ cingulate cingulum, $\mathrm{TCing}=$ temporal cingulum, $\mathrm{FAT}=$ frontal aslant tract, $F x=$ fornix, IFOF = inferior fronto-occipital fasciculu, ILF = inferior longitudinal fasciculus, MdLF = middle longitudinal fasciculus, SLF = superior longitudinal fasciculus, SLF-IId = SLF-II dorsal division, SLF-IIv = SLF-II ventral division, UF = uncinate fasciculus, VOF = vertical occipital fasciculus, $\mathrm{ML}=$ medial lemniscus, $\mathrm{OR}=$ optic radiation, occlobe = occipital lobe, $\mathrm{OT}=$ optic tract, $\mathrm{PyT}=$ pyramidal tract, $\mathrm{CST}=$ corticospinal tract, $\mathrm{M} 1$ = primary motor cortex, $\mathrm{PyT}=$ pyramidal tract, $\mathrm{PMC}=$ premotor cortex, SMA = supplementary motor area, $\mathrm{ThR}=$ thalamic radiation, Ant $=$ anterior, Par $=$ parietal, Sup $=$ superior, Ant Comm $=$ anterior commissure, $C C=$ corpus callosum, PMC and SMA = premotor cortex and supplementary motor area, DRT = dentatorubro-thalamic tract, $\mathrm{ICP}=$ inferior cerebellar peduncle, $\mathrm{MCP}=$ middle cerebellar peduncle, $\mathrm{L}=\mathrm{left}$, $\mathrm{R}=$ right. 
bioRxiv preprint doi: $h$ ttps://doi org/10.1101/2021 10.13464139. this version posted October 19, 2021. The copyright holder for this preprint (which was not certified by peer review) is the author/funder, who has granted bioRxiv a license to display the preprint in perpetuity. It is made available under aCC-BY 4.0 International license. agreement in bundle cores and maximum variability around the periphery.
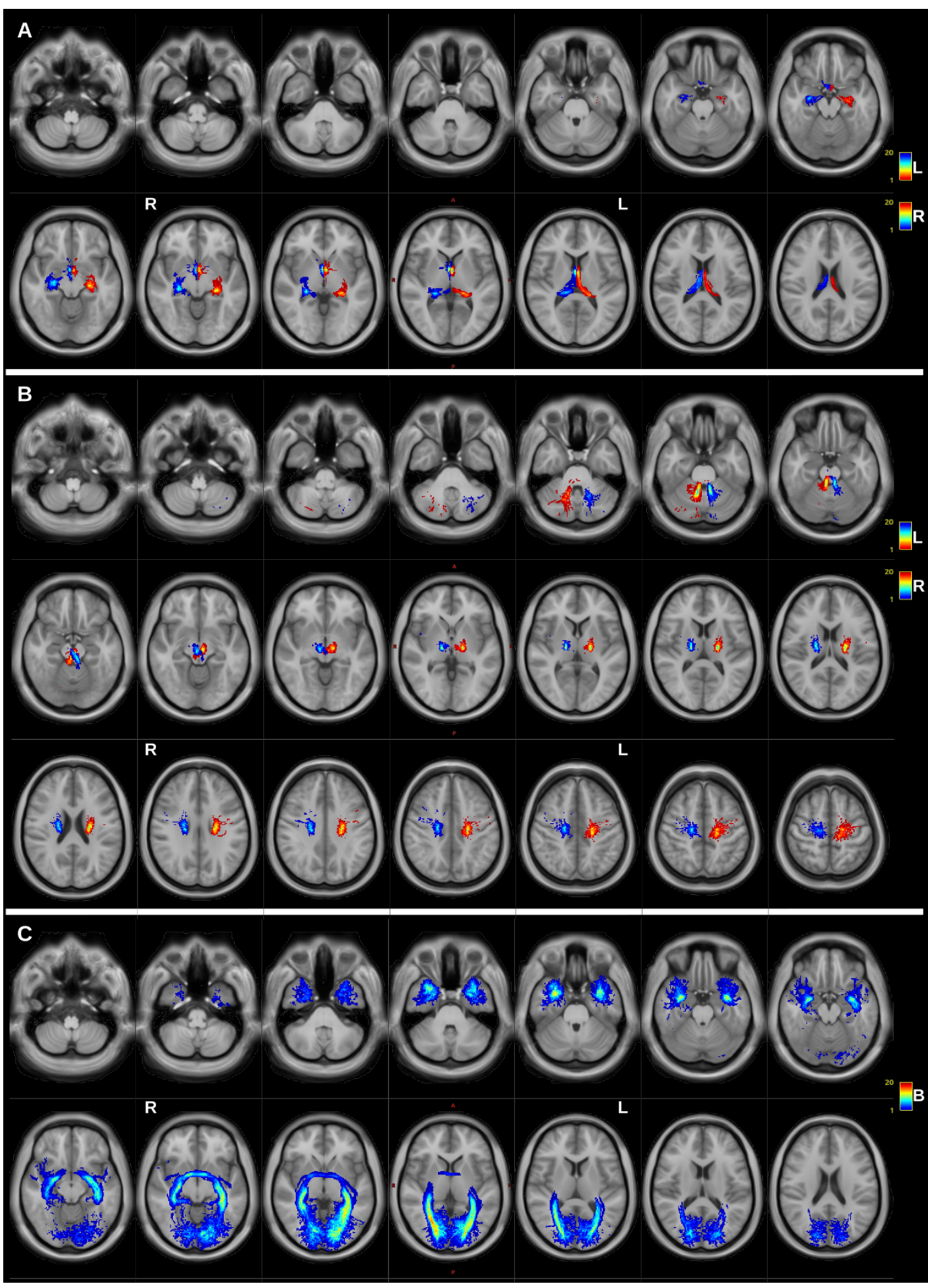

Figure 21: Serial axial slices of the HCP-template T1-weighted images with the cumulative voxelwise heat-maps of the fornix $(A)$, dentato-rubro-thalamic tracts $(B)$, and anterior commissure $(C), L$ $=$ left, $\mathrm{R}=$ right, $\mathrm{B}=$ bilateral. Left sided bundles are shown in red-to-blue, and right sided bundles are shown in blue-to-red. 
bioRxiv preprint doi: https://doi.org/10.1101/2021 10.13464139; this version posted October 19, 2021. The copyright holder for this preprint (which was not certified by peer review) is the author/funder, who has granted bioRxiv a license to display the preprint in perpetuity. It is made available under aCC-BY 4.0 International license.

591

592

593

594

Figures $21 \& 22$ demonstrate these maps for the AF and CST, and fornix and DRTT, respectively as examples of some of the best and worst performing bundles in terms of similarity to HCP-template and inter-session reproducibility. Equivalent maps are provided for all other bundles in the supplementary material.
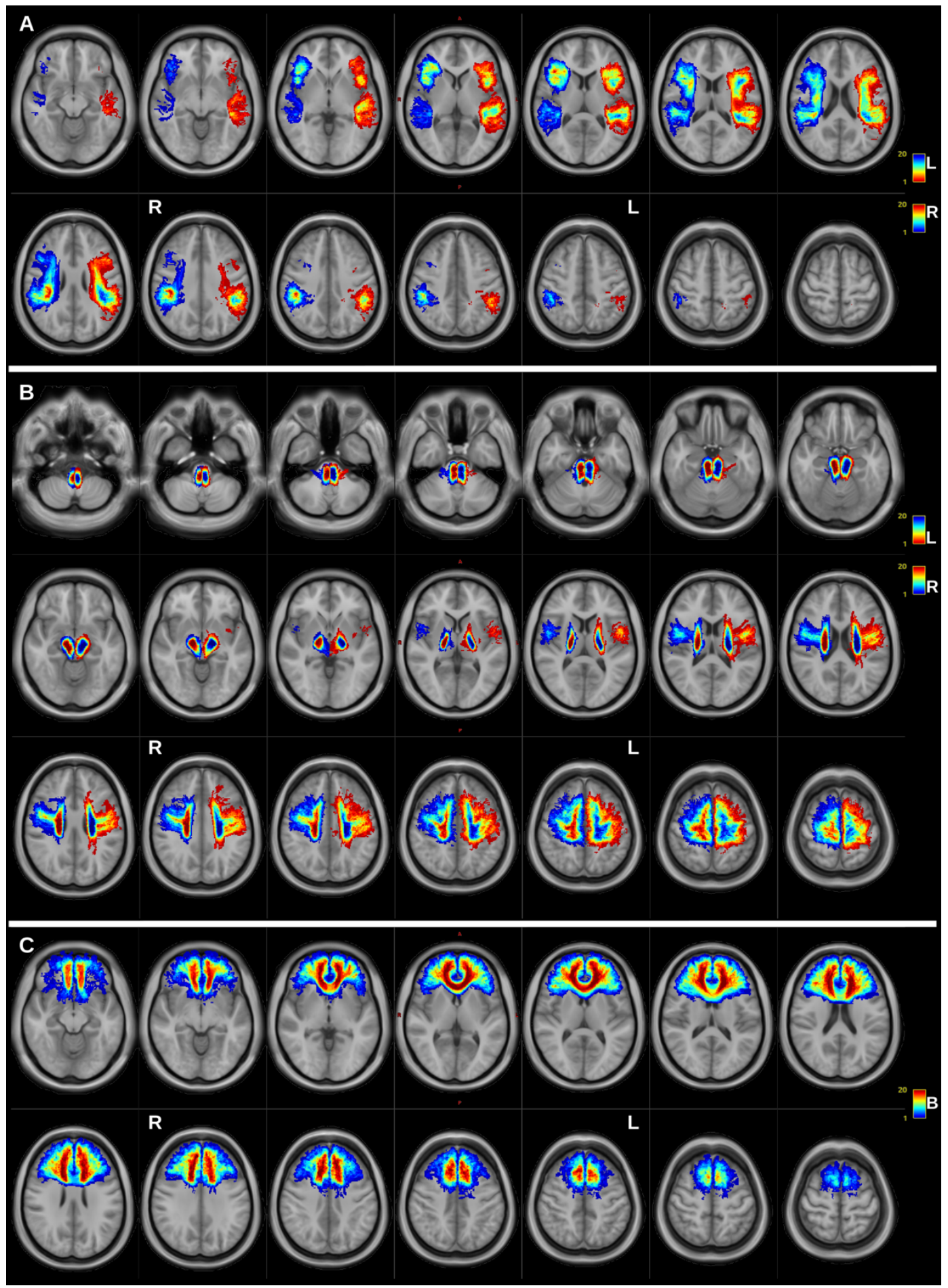

Figure 22: Serial axial slices of the HCP-template T1-weighted images with the cumulative voxelwise heat-maps of the arcuate fasciculi $(A)$, whole pyramidal tracts $(B)$, and prefrontal corpus callosum $(C), L=$ left, $R=$ right, $B=$ bilateral. Left side bundles are shown in red-to-blue, and right sided bundles are shown in blue-to-red. 


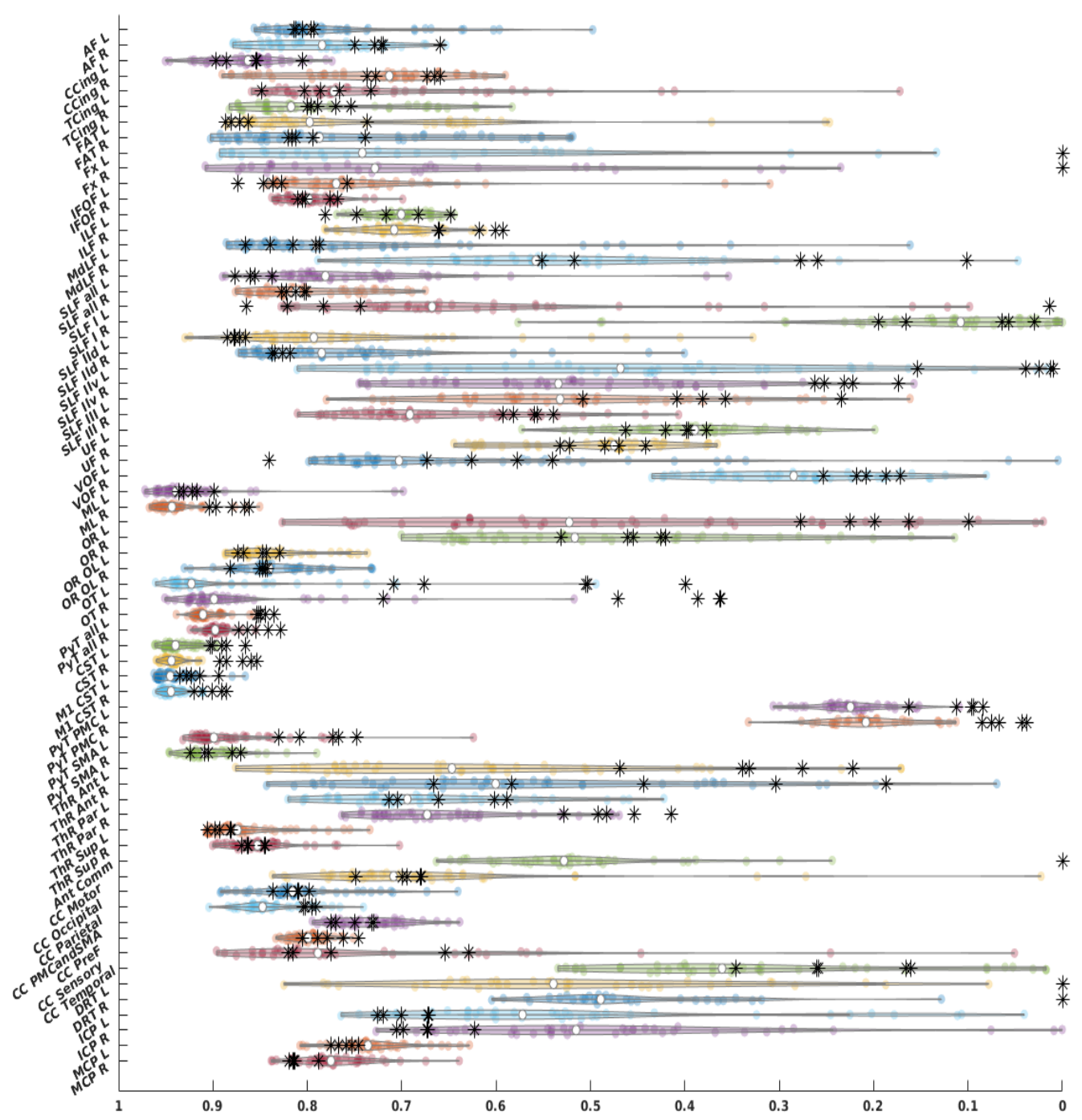

Figure 23:Weighted dice similarity coefficient scores for all bundles, HCP test-retest tractograms are depicted as violin plots and MASSIVE results are depicted as black asterisks., AF = arcuate fasciculus, CCing $=$ cingulate cingulum, TCing $=$ temporal cingulum, FAT $=$ frontal aslant tract, $F x=$ fornix, IFOF = inferior fronto-occipital fasciculu, ILF = inferior longitudinal fasciculus, MdLF = middle longitudinal fasciculus, SLF = superior longitudinal fasciculus, SLF-IId = SLF-II dorsal division, SLF-IIv = SLF-II ventral division, UF $=$ uncinate fasciculus, $V O F=$ vertical occipital fasciculus, $M L=$ medial lemniscus, $\mathrm{OR}=$ optic radiation, occlobe = occipital lobe, $\mathrm{OT}=$ optic tract, $\mathrm{PyT}=$ pyramidal tract, $\mathrm{CST}$ = corticospinal tract, $\mathrm{M} 1$ = primary motor cortex, $\mathrm{PyT}=$ pyramidal tract, $\mathrm{PMC}=$ premotor cortex, $\mathrm{SMA}$ = supplementary motor area, $\mathrm{ThR}=$ thalamic radiation, $\mathrm{Ant}=$ anterior, $\mathrm{Par}=$ parietal, Sup $=$ superior, Ant Comm = anterior commissure, $\mathrm{CC}=$ corpus callosum, $\mathrm{PMC}$ and SMA = premotor cortex and supplementary motor area, DRT = dentato-rubro-thalamic tract, $\mathrm{ICP}=$ inferior cerebellar peduncle, $M C P=$ middle cerebellar peduncle,$L=$ left, $R=$ right.

In total we successfully reconstructed 316 out of 340 bundles (68 for each of the 5

597 MASSIVE datasets). 63 out of the 68 bundles were generated for every dataset, while

598 the anterior commissure was only generated in 1 dataset, and the DRTT and fornix

599 were not generated in any dataset. Figure 23 shows the wDSC scores derived from 
600

601

602

603

604

605

606

607

608

609

610

611

612

613

614

615

616

618

comparing the MASSIVE bundles to the HCP-template bundles superimposed on the results of the HCP test-retest dataset for reference. The single score agreement ICC score was 0.917 (upper-bound $=0.944$, lower-bound $=0.884, p<0.05$ ).

\section{Discussion}

Human WM segmentation based on diffusion imaging tractography has been likened to blurring the boundary between science and art (Van Hecke et al., 2016). While few would argue about the aesthetic qualities of these virtual dissections, when applied appropriately, they transcend pretty pictures to become an indispensable tool in modern neurosurgical practice and neuroscience research (Johansen-Berg and Behrens, 2006; Panesar et al., 2019). In this work we aim to facilitate the use of advanced tractography methods within the clinical research community. First, we provide an intuitive educational reference for researchers and clinicians wishing to apply CSD tractography to typical HARDI datasets. Second, we describe typical WM anatomy derived from automated subject-specific parcellation driven CSD probabilistic tractography in adults. Third, we demonstrate the variability of the reconstructed bundles within and between subjects using two test-retest datasets and offer solutions to improve tracking results for the least reproducible bundles. Finally, we provide an open-source fully-automated and customizable tractography pipeline (FWT) enabling researchers and clinicians to apply the methods presented in this work to their own data.

\subsection{Variability of virtual dissections}

Our results demonstrate that inter-bundle variability was much greater than intersubject variability for the same bundles i.e., there was a wide range of similarity in the extent to which different bundles were reconstructed irrespective of the dataset used. The AF and PyT for example were reconstructed in largely the same way in all datasets, whereas the fornix and DRTT showed high variability in density and spatial extent and were sometimes not reconstructed at all. In line with this, inter-subject agreement was highest for bundles with high streamline densities e.g., PyT, AF, CC and lowest for bundles with low streamline densities e.g., fornix, and DRTT. 
Inter-session and intra-subject agreement were high for all bundles i.e., bundles were reconstructed largely to the same extent, (or failed to be reconstructed) in both sessions of the HCP test-retest dataset. In the test-retest data from a single-subject with varying b-values and shell schemes (MASSIVE) changing the b-value(s), number of gradient directions or shell scheme did not consistently alter bundle similarity to the model.

These findings align with a large body of evidence that diffusion tractography reconstructions are highly variable and are influenced not only by data acquisition parameters such as scanner manufacturer and field strength, b-value, diffusion sampling scheme, reconstruction model (Schilling et al., 2021), but also by the virtual dissection protocol itself (Schilling et al., 2020). Notably, in the context of our study, despite using exactly the same WM dissection protocol for the HCP-template as for the individual datasets, none of the tracked bundles failed in the template data. This highlights the importance of data quality, as group-averaged data tends to have a much higher signal-to-noise ratio (SNR) despite increased blurriness in fine cortical structures due to inter-subject variability. It also demonstrates that even in a unified fully automated WM dissection protocol considerable inter-bundle and inter-subject variability remain. In contrast to previous work, we found that data acquisition parameters and sampling scheme had only a limited impact on bundle variability. This is likely due to the high number of volumes used per session, which would result in a higher SNR compared to the typical single acquisition datasets used in comparison studies.

In agreement with other studies (Bonilha et al., 2015; Cousineau et al., 2017; Gu et al., 2017; Zhang et al., 2019), we found that larger bundles, particularly if they have both a larger volume and a higher streamline count, tend to be more similar/reproducible regardless of differences in scanning parameters or even normal inter-subject anatomical variations. It follows that automated procedures are most appropriate for fasciculi with these characteristics. For smaller, less dense or more narrow bundles such as the fornix, DRTT (and relatedly, the superior cerebellar peduncle) or specific subdivisions of larger bundles (eg SLF-I, temporal corpus callosum) we recommend using either data with sub-millimeter spatial resolution, bundle-specific manual dissection or using the template bundles we provide in an 
661 automated in a RecoBundles (Garyfallidis et al., 2018) based workflow rather than the

662 VOI-based approach. Of note, we also provide symmetrical versions of the HCP.

663 template bundles along with aligned reference anatomical images. In line with the use

664 of template bundles, FWT is compatible with RecoBundles (Garyfallidis et al., 2018)

665 based pipelines that can overcome the need for structural parcellation, which can be

666 particularly useful in clinical data to avoid false tractography results due to structural

parcellation errors.

\subsection{The benefits of using a CSD atlas in clinical studies}

669

670

671

672

673

674

675

676

677

678

679

680

681

682

683

684

685

686

687

688

689

690

There are currently a number of diffusion-based tractography atlases and protocols available (Catani and Thiebaut de Schotten, 2008; Thiebaut de Schotten et al., 2011; Verhoeven et al., 2010; Wakana et al., 2004; Warrington et al., 2020; Wasserthal et al., 2018; Yendiki et al., 2011) some of which are based on HCP data (Hansen et al., 2020; Yeh et al., 2018). Here we aimed to primarily address the limitations of DTI based virtual dissection protocols, which predominate in clinical studies, either as part of comparative studies with healthy controls, or in a healthcare setting using data acquired in shorter scan times than are achievable in a research environment. We did not include formal comparison to other HARDI/higher-order modelling-based atlases which precludes us from drawing conclusions about the relative benefits of FWT over other methods for virtual dissection in this context. It is our aim to complement atlases based on detailed anatomical research and specialized acquisition schemes with a comprehensive description of CSD-based human WM anatomy which improves upon classical DTI representations in 3T data. Whilst the results presented here are based on high quality data in healthy subjects, we also tested our protocol on lower quality, neurosurgical patient data during the development phase of FWT, to ensure its translatability to clinical populations. A formal analysis relating to the application of FWT in patients with lesions is beyond the scope of this paper focusing on typical CSD anatomy and will be presented in a follow-up publication.

\subsection{Technical considerations and limitations}

This work relied on imaging data from only 20 individuals, however by using re-test scans yielding 40 datasets and the additional analysis using the MASSIVE dataset, 
691 our sample is sufficient to study test-retest reproducibility, as well as inter-subject 692 variability. While our dissection protocol was literature-based and guided by several 693 contemporary publications we did not include a direct comparison to any other atlas, 694 nor did we apply formal specific criteria for assessing the validity of the definitions. 695 Given the lack of consensus on anatomical definitions relating to WM fasciculi, it is possible that some readers will disagree with our interpretation, and that the FWT VOIs and reconstructions may need to be revised as the field evolves.

We attempted to keep rigid heuristic decisions (e.g., statistical thresholds) to a minimum especially those pertaining to streamlines filtering, where we utilized largely

700

701

702 data-driven streamline filtering tools from Dipy (Garyfallidis et al., 2014) rather than relying on a more stringent selection of anatomical exclude VOls. However, in order to cater to varying data quality, our choice of inclusion VOls in some situations are influenced by considerations made for lower quality datasets than the ones utilized in this work. For example, for the AF we excluded any streamlines involving the precentral gyrus to avoid false streamlines tunneling through volume average voxels, which we have observed in lower quality data single-shell data. Similarly, the AC is generated without the olfactory component, and we do not include subdivisions to the AF, CST, full posterior thalamic radiation, or superior cerebellar peduncles proper. We also do not include more esoteric bundles such as the superior-anterior fasciculus (David et al., 2019), temporo-insular fasciculus (Nachtergaele et al., 2019; Radwan et al., 2019), and inferior (auditory) thalamic radiation (Maffei et al., 2019a, 2019b), or those with a high chance of failure due to their geometry, such as the posterior commissure, or cranial nerves.

FWT tractography utilizes spherical-deconvolution informed filtering of tractograms (SIFT) (Smith et al., 2015, 2013)) based streamlines seeding and filtering for whole brain tractography, while further improvement can be expected if an optimized whole brain tractography method is utilized, e.g., global tractography (Christiaens et al., 2015) or particle-filtering tractography (Girard et al., 2014). FWT does not use a specific required number of output streamlines during bundle segmentation from whole-brain tractograms to avoid biasing the outcome by an expected number of streamlines. However, this is unavoidable if the bundle specific approach is utilized. In this case the resulting bundle will be directly influenced by the required number of 
723 streamlines as this will influence the number of streamlines seeding attempts. This

724 point is illustrated in supplementary figure 1, which shows that when the DRT template

725 bundles are generated twice with 2,000 and 50,000 required streamlines initially, the

726 outcome from the 50,000 streamlines appears more complete as can be expected.

727 Furthermore, when applied to the individual datasets, the bundle-specific approach

728 with 20,000 required streamlines reconstructed more bundles successfully than the

729 whole-brain tractography and bundle segmentation approach (73 vs. 67), and resulted

730 in higher wDSC scores particularly when compared to the 50,000 streamlines DRT

731 template bundles.

732 The FWT pipeline is time-consuming, as FS recon-all alone requires at least 4 - 6

733 hours and dMRI preprocessing can vary widely depending on acquisition parameters,

734 and reconstruction method. E.g., whole brain tractography can take more than 4 hours

735 for a multi-shell multi-tissue CSD model with probabilistic tractography using second-

736 order integration over distributions of fODFs (iFOD2) (Tournier et al., 2010) with fODF-

737 driven dynamic seeding (Smith et al., 2015). Whilst this is offset by the flexibility of

738 FWT allowing a more targeted approach using bundle-specific seeding and

739 tractography in case of presurgical mapping, further development will focus on

740 improving processing time. Additionally, future work will provide a clustering-based

741 workflow independent of prior structural parcellation, the addition of more fiber

742 bundles, as well as generating deterministic versions of all bundles. Finally, any

743 justified and necessary changes to the inclusion/exclusion VOls for any bundle can be

744 easily implemented by changing the FWT workflows, which are openly provided, and

745 a new atlas of bundles can be easily created by rerunning the template workflows on

746 the HCP-group template data.

\section{Conclusion}

748 The FWT pipeline can reconstruct 68 WM fasciculi and shows high inter- and intra749 subject reproducibility. Dense bundles such as the PyT, AF and MCP yield the most 750 reliable and least variable reconstructions. Higher resolution data or tract specific 751 modification may be required for thinner bundles such as the fornix, DRTT, SLF I and 752 premotor PyT. The FWT CSD atlas may be a useful reference and virtual dissection 
753 tool for applied neuroimaging students and clinical professionals wishing to use and

754 understand the capabilities of CSD tractography.

\section{Acknowledgments and disclosures}

756

757

758

759

760

761

762

763

764

765

766

767

768

769

770

771

772 (https://github.com/KUL-Radneuron/KUL_FWT.git). Finally, the Open Science

773 Framework repository (https://osf.io/snq2d/) contains additional screenshots of single

774 subject bundles, bundle heatmaps, original and symmetric versions of the HCP-

775 template atlas bundles, and additional tractograms. 
$\mathbf{A C}=$ Anterior Commissure

$\mathrm{AC} / \mathrm{PC}=$ Anterior Commissure/Posterior

Commissure

$\mathbf{A F}=$ Arcuate Fasciculus

$\mathbf{A P}=$ Anterior-Posterior

ATR = Anterior Thalamic Radiation

BIDS = Brain Imaging Data Structure

CC $=$ Corpus Callosum

CG = Cingulum

CSD = Constrained Spherical Deconvolution

CST $=$ CorticoSpinal Tract

DL = DorsoLateral nucleus of the thalamus

DM = DorsoMedial nucleus of the thalamus

dMRI = Diffusion Magnetic Resonance

imaging

DRTT = Dentato-Rubro-Thalamic Tract

DSC = Dice Similarity Coefficient

DSI = Diffusion Spectral Imaging

DTI = Diffusion Tensor Imaging

$\mathbf{F A}=$ Fractional Anisotropy

FACT = Fiber Assignment by Continuous

Tracking

FAT = Frontal Aslant Tract

fODF = Fiber Orientation Distribution

Function

$\mathbf{F S}=$ FreeSurfer

FT = Fiber Tracking/Tractography

FWT = Fun With Tracts

Fx $=$ Fornix

HARDI = High Angular Resolution Diffusion Imaging

HCP = Human Connectome Project

ICC = Intra-Class Correlation

ICP = Inferior Cerebellar Peduncle

IFOF = Inferior Fronto-Occipital Fasciculus

ILF = Inferior Longitudinal Fasciculus

M1 = Primary Motor cortex
MALP-EM = Multi-Atlas Label

Propagation with Expectation

Maximization

MASSIVE = Multiple Acquisitions for

Standardization of Structural Imaging

Validation and Evaluation

MCP = Middle Cerebellar Peduncle

MdLF $=$ Middle Longitudinal Fasciculus

ML = Medial Lemniscus

MR = Magnetic Resonance

MRI = Magnetic Resonance Imaging

MSBP = MultiScale Brain Parcellator

NIST $=$ Neuroimaging and Surgical

Technologies

OR = Optic Radiation

OT = Optic Tract

PaTR = Parietal Thalamic Radiation

PD25 = Parkinson's Disease 25

subjects histological atlas

PoTR = Posterior Thalamic Radiation

PyT = Pyramidal Tract

ROIs $=$ Regions Of Interest

SLF = Superior Longitudinal Fasciculus

SNR = Signal-to-Noise Ratio

STR = Superior Thalamic Radiation

SUIT = Spatially Unblased atlas

Template of the cerebellum and

brainstem

UF = Uncinate Fasciculus

UKBB = United Kingdom BioBank

VOF $=$ Vertical Occipital Fasciculus

VOls $=$ Volumes of interest

VPL $=$ Ventral Postero-Lateral

VPM $=$ Ventral Postero-Medial

wDSC $=$ Weighted Dice Similarity

Coefficient

$\mathbf{W M}=$ White Matter 


\section{References}

1- Almairac, F., Herbet, G., Moritz-Gasser, S., de Champfleur, N.M., Duffau, H., 2015. The left inferior fronto-occipital fasciculus subserves language semantics: a multilevel lesion study. Brain Struct. Funct. 220, 1983-1995. https://doi.org/10.1007/s00429-014-0773-1

2- Andersson, J.L.R., Skare, S., Ashburner, J., 2003a. How to correct susceptibility distortions in spin-echo echo-planar images: application to diffusion tensor imaging. Neurolmage 20, 870-888. https://doi.org/10.1016/S10538119(03)00336-7

3- Andersson, J.L.R., Skare, S., Ashburner, J., 2003b. How to correct susceptibility distortions in spin-echo echo-planar images: application to diffusion tensor imaging. Neurolmage 20, 870-888. https://doi.org/10.1016/S10538119(03)00336-7

4- Andersson, J.L.R., Sotiropoulos, S.N., 2016. An integrated approach to correction for off-resonance effects and subject movement in diffusion MR imaging. Neurolmage 125, 1063-1078. https://doi.org/10.1016/j.neuroimage.2015.10.019

5- Andersson, J.L.R., Sotiropoulos, S.N., 2015. Non-parametric representation and prediction of single- and multi-shell diffusion-weighted MRI data using Gaussian processes. Neurolmage 122, 166-176. https://doi.org/10.1016/j.neuroimage.2015.07.067

6- Avants, B.B., Tustison, N.J., Song, G., Cook, P.A., Klein, A., Gee, J.C., Gee, C., 2011. A Reproducible Evaluation of ANTs Similarity Metric Performance in Brain Image Registration. Neuroimage. 54, 2033-2044. https://doi.org/10.1016/j.neuroimage.2010.09.025.A

7- Bain, J.S., Yeatman, J.D., Schurr, R., Rokem, A., Mezer, A.A., 2019. Evaluating arcuate fasciculus laterality measurements across dataset and tractography pipelines. Hum. Brain Mapp. 40, 3695-3711. https://doi.org/10.1002/hbm.24626

8- Barbeau, E.B., Descoteaux, M., Petrides, M., 2020. Dissociating the white matter tracts connecting the temporo-parietal cortical region with frontal cortex 
using diffusion tractography. Sci. Rep. $10, \quad 8186$. https://doi.org/10.1038/s41598-020-64124-y

9- Basser, P.J., Mattiello, J., LeBihan, D., 1994a. MR diffusion tensor spectroscopy and imaging. Biophys. J. 66, 259-267. https://doi.org/10.1016/S00063495(94)80775-1

10-Basser, P.J., Mattiello, J., LeBihan, D., 1994b. Estimation of the effective selfdiffusion tensor from the NMR spin echo. J. Magn. Reson. B 103, 247-254. https://doi.org/10.1006/jmrb.1994.1037

11-Bastiani, M., Cottaar, M., Fitzgibbon, S.P., Suri, S., Alfaro-Almagro, F., Sotiropoulos, S.N., Jbabdi, S., Andersson, J.L.R., 2019. Automated quality control for within and between studies diffusion MRI data using a non-parametric framework for movement and distortion correction. Neurolmage 184, 801-812. https://doi.org/10.1016/j.neuroimage.2018.09.073

12-Bayrak, R.G., Wang, X., Schilling, K.G., Greer, J.M., Hansen, C.B., Blaber, J.A., Williams, O., Beason-Held, L.L., Resnick, S.M., Rogers, B.P., Landman, B.A., 2020. TractEM: Fast Protocols for Whole Brain Deterministic TractographyBased White Matter Atlas. https://doi.org/10.1101/651935

13-Behrens, T.E.J., Johansen-Berg, H., Woolrich, M.W., Smith, S.M., WheelerKingshott, C. a. M., Boulby, P.A., Barker, G.J., Sillery, E.L., Sheehan, K., Ciccarelli, O., Thompson, A.J., Brady, J.M., Matthews, P.M., 2003. Non-invasive mapping of connections between human thalamus and cortex using diffusion imaging. Nat. Neurosci. 6, 750-757. https://doi.org/10.1038/nn1075

14-Bernard, F., Zemmoura, I., Ter Minassian, A., Lemée, J.-M., Menei, P., 2019. Anatomical variability of the arcuate fasciculus: a systematical review. Surg. Radiol. Anat. 41, 889-900. https://doi.org/10.1007/s00276-019-02244-5

15-Bloy, L., Ingalhalikar, M., Eavani, H., Schultz, R.T., Roberts, T.P.L., Verma, R., 2012. White matter atlas generation using HARDI based automated parcellation. Neurolmage 59, 4055-4063. https://doi.org/10.1016/j.neuroimage.2011.08.053

16-Bonilha, L., Gleichgerrcht, E., Fridriksson, J., Rorden, C., Breedlove, J.L., Nesland, T., Paulus, W., Helms, G., Focke, N.K., 2015. Reproducibility of the 
Structural Brain Connectome Derived from Diffusion Tensor Imaging. PLOS ONE 10, e0135247. https://doi.org/10.1371/journal.pone.0135247

17-Bore, A., Rheault, F., Theaud, G., Théberge, A., 2021. Scilpy [WWW Document]. URL https://github.com/scilus/scilpy (accessed 8.3.21).

18-Bosch-Bouju, C., Hyland, B.I., Parr-Brownlie, L.C., 2013. Motor thalamus integration of cortical, cerebellar and basal ganglia information: implications for normal and parkinsonian conditions. Front. Comput. Neurosci. 7. https://doi.org/10.3389/fncom.2013.00163

19-Bouyagoub, S., Dowell, N.G., Gabel, M., Cercignani, M., 2020. Comparing multiband and singleband EPI in NODDI at $3 \mathrm{~T}$ : what are the implications for reproducibility and study sample sizes? Magn. Reson. Mater. Phys. Biol. Med. https://doi.org/10.1007/s10334-020-00897-7

20-Calamuneri, A., Arrigo, A., Mormina, E., Milardi, D., Cacciola, A., Chillemi, G., Marino, S., Gaeta, M., Quartarone, A., 2018. White Matter Tissue Quantification at Low b-Values Within Constrained Spherical Deconvolution Framework. Front. Neurol. 9, 716-716. https://doi.org/10.3389/fneur.2018.00716

21-Catani, M., 2006. Diffusion tensor magnetic resonance imaging tractography in cognitive disorders. Curr. Opin. Neurol. 19, 599-606. https://doi.org/10.1097/01.wco.0000247610.44106.3f

22-Catani, M., Howard, R.J., Pajevic, S., Jones, D.K., 2002. Virtual in Vivo Interactive Dissection of White Matter Fasciculi in the Human Brain. Neurolmage 17, 77-94. https://doi.org/10.1006/nimg.2002.1136

23-Catani, M., Thiebaut de Schotten, M., 2008. A diffusion tensor imaging tractography atlas for virtual in vivo dissections. Cortex, Special Issue on "Brain Hodology - Revisiting disconnection approaches to disorders of cognitive function" 44, 1105-1132. https://doi.org/10.1016/j.cortex.2008.05.004

24-Çavdar, S., Aydın, A.E., Algın, O., Aydın, S., 2021. The Complex Structure of the Anterior White Commissure of the Human Brain: Fiber Dissection and Tractography Study. World Neurosurg. 147, e111-e117. https://doi.org/10.1016/j.wneu.2020.11.157

25-Caverzasi, E., Hervey-Jumper, S.L., Jordan, K.M., Lobach, I.V., Li, J., Panara, V., Racine, C.A., Sankaranarayanan, V., Amirbekian, B., Papinutto, N., Berger, 
M.S., Henry, R.G., 2016. Identifying preoperative language tracts and predicting postoperative functional recovery using HARDI q-ball fiber tractography in patients with gliomas. J. Neurosurg. 125, 33-45. https://doi.org/10.3171/2015.6.JNS142203

26-Chamberland, M., Tax, C.M.W., Jones, D.K., 2018. Meyer's loop tractography for image-guided surgery depends on imaging protocol and hardware. Neurolmage Clin. 20, 458-465. https://doi.org/10.1016/j.nicl.2018.08.021

27-Chen, Z., Tie, Y., Olubiyi, O., Rigolo, L., Mehrtash, A., Norton, I., Pasternak, O., Rathi, Y., Golby, A.J., O'Donnell, L.J., 2015. Reconstruction of the arcuate fasciculus for surgical planning in the setting of peritumoral edema using twotensor unscented Kalman filter tractography. Neurolmage Clin. 7, 815-822. https://doi.org/10.1016/j.nicl.2015.03.009

28-Chenot, Q., Tzourio-Mazoyer, N., Rheault, F., Descoteaux, M., Crivello, F., Zago, L., Mellet, E., Jobard, G., Joliot, M., Mazoyer, B., Petit, L., 2019. A population-based atlas of the human pyramidal tract in 410 healthy participants. Brain Struct. Funct. 224, 599-612. https://doi.org/10.1007/s00429-018-1798-7 29-Choi, H., Kubicki, M., Whitford, T., Alvarado, J.L., Terry, D.P., Niznikiewicz, M., McCarley, R.W., Kwon, J.S., Shenton, M.E., 2011. Diffusion Tensor Imaging of Anterior Commissural Fibers in Patients with Schizophrenia. Schizophr. Res. 130, 78-85. https://doi.org/10.1016/j.schres.2011.04.016

30-Christiaens, D., Reisert, M., Dhollander, T., Sunaert, S., Suetens, P., Maes, F., 2015. Global tractography of multi-shell diffusion-weighted imaging data using a multi-tissue model. Neurolmage 123, 89-101. https://doi.org/10.1016/j.neuroimage.2015.08.008

31-Christiansen, K., Metzler-Baddeley, C., Parker, G.D., Muhlert, N., Jones, D.K., Aggleton, J.P., Vann, S.D., 2017. Topographic separation of fornical fibers associated with the anterior and posterior hippocampus in the human brain: An MRI-diffusion study. Brain Behav. 7, e00604. https://doi.org/10.1002/brb3.604 32-Coenen, V.A., Sajonz, B., Prokop, T., Reisert, M., Piroth, T., Urbach, H., Jenkner, C., Reinacher, P.C., 2020. The dentato-rubro-thalamic tract as the potential common deep brain stimulation target for tremor of various origin: an 
observational case series. Acta Neurochir. (Wien) 162, 1053-1066. https://doi.org/10.1007/s00701-020-04248-2

33-Cordero-Grande, L., Christiaens, D., Hutter, J., Price, A.N., Hajnal, J.V., 2019. Complex diffusion-weighted image estimation via matrix recovery under general noise models. Neurolmage 200, 391-404. https://doi.org/10.1016/j.neuroimage.2019.06.039

34-Cousineau, M., Jodoin, P.-M., Garyfallidis, E., Côté, M.-A., Morency, F.C., Rozanski, V., Grand'Maison, M., Bedell, B.J., Descoteaux, M., 2017. A testretest study on Parkinson's PPMI dataset yields statistically significant white matter fascicles. Neurolmage Clin. 16, 222-233. https://doi.org/10.1016/j.nicl.2017.07.020

35-David, S., Heemskerk, A.M., Corrivetti, F., Thiebaut de Schotten, M., Sarubbo, S., Corsini, F., De Benedictis, A., Petit, L., Viergever, M.A., Jones, D.K., Mandonnet, E., Axer, H., Evans, J., Paus, T., Leemans, A., 2019. The Superoanterior Fasciculus (SAF): A Novel White Matter Pathway in the Human Brain? Front. Neuroanat. 0. https://doi.org/10.3389/fnana.2019.00024

36-de Schotten, M.T., Dell'Acqua, F., Forkel, S.J., Simmons, A., Vergani, F., Murphy, D.G.M., Catani, M., 2011. A lateralized brain network for visuospatial attention. Nat. Neurosci. 14, 1245-1246. https://doi.org/10.1038/nn.2905

37-De Witt Hamer, P.C., Moritz-Gasser, S., Gatignol, P., Duffau, H., 2010. Is the human left middle longitudinal fascicle essential for language? A brain electrostimulation study. Hum. Brain Mapp. 32, 962-973. https://doi.org/10.1002/hbm.21082

38-Dick, A.S., Garic, D., Graziano, P., Tremblay, P., 2019. The frontal aslant tract (FAT) and its role in speech, language and executive function. Cortex J. Devoted Study Nerv. Syst. Behav. 111, 148-163. https://doi.org/10.1016/j.cortex.2018.10.015

39-Dick, A.S., Tremblay, P., 2012. Beyond the arcuate fasciculus: consensus and controversy in the connectional anatomy of language. Brain 135, 3529-3550. https://doi.org/10.1093/brain/aws222 
40-Diedrichsen, J., 2006. A spatially unbiased atlas template of the human cerebellum.

Neurolmage

33 ,

127-138.

https://doi.org/10.1016/j.neuroimage.2006.05.056

41-Diedrichsen, J., Balsters, J.H., Flavell, J., Cussans, E., Ramnani, N., 2009. A probabilistic MR atlas of the human cerebellum 8.

42-Diedrichsen, J., Maderwald, S., Küper, M., Thürling, M., Rabe, K., Gizewski, E.R., Ladd, M.E., Timmann, D., 2011. Imaging the deep cerebellar nuclei: A probabilistic atlas and normalization procedure. Neurolmage 54, 1786-1794. https://doi.org/10.1016/j.neuroimage.2010.10.035

43-Diedrichsen, J., Zotow, E., 2015. Surface-Based Display of Volume-Averaged Cerebellar Imaging Data. PLOS ONE 10, e0133402. https://doi.org/10.1371/journal.pone.0133402

44-Duan, F., Zhao, T., He, Y., Shu, N., 2015. Test-retest reliability of diffusion measures in cerebral white matter: A multiband diffusion MRI study. J. Magn. Reson. Imaging 42, 1106-1116. https://doi.org/10.1002/jmri.24859

45-Fabri, M., Pierpaoli, C., Barbaresi, P., Polonara, G., 2014. Functional topography of the corpus callosum investigated by DTI and fMRI. World J. Radiol. 6, 895-906. https://doi.org/10.4329/wjr.v6.i12.895

46-Farquharson, S., Tournier, J.-D., Calamante, F., Fabinyi, G., Schneider-Kolsky, M., Jackson, G.D., Connelly, A., 2013. White matter fiber tractography: why we need to move beyond DTI. J. Neurosurg. 118, 1367-1377. https://doi.org/10.3171/2013.2.JNS121294

47-Fernández-Miranda, J.C., Wang, Y., Pathak, S., Stefaneau, L., Verstynen, T., Yeh, F.C., 2015. Asymmetry, connectivity, and segmentation of the arcuate fascicle in the human brain. Brain Struct. Funct. 220, 1665-1680. https://doi.org/10.1007/s00429-014-0751-7

48-Fischl, B., 2012. FreeSurfer. Neurolmage 62, 774-781. https://doi.org/10.1016/j.neuroimage.2012.01.021

49-Forkel, S.J., Thiebaut de Schotten, M., Kawadler, J.M., Dell'Acqua, F., Danek, A., Catani, M., 2014. The anatomy of fronto-occipital connections from early blunt dissections to contemporary tractography. Cortex, The clinical 
neuroanatomy of the occipital lobes $56,73-84$.

https://doi.org/10.1016/j.cortex.2012.09.005

51-Froeling, M., Tax, C.M.W., Vos, S.B., Luijten, P.R., Leemans, A., 2017.

"MASSIVE" brain dataset: Multiple acquisitions for standardization of structural imaging validation and evaluation. Magn. Reson. Med. 77, 1797-1809. https://doi.org/10.1002/mrm.26259

52-Garyfallidis, E., Brett, M., Amirbekian, B., Rokem, A., Van Der Walt, S., Descoteaux, M., Nimmo-Smith, I., 2014. Dipy, a library for the analysis of

53-Garyfallidis, E., Brett, M., Correia, M.M., Williams, G.B., Nimmo-Smith, I., 2012. QuickBundles, a Method for Tractography Simplification. Front. Neurosci. 6. https://doi.org/10.3389/fnins.2012.00175

54-Garyfallidis, E., Côté, M.-A., Rheault, F., Sidhu, J., Hau, J., Petit, L., Fortin, D., local and global streamline-based registration and clustering. Neurolmage,

$$
\begin{array}{llll}
\text { Segmenting } \quad \text { Brain 283-295. } & \text { 170, }
\end{array}
$$

$$
\text { https://doi.org/10.1016/j.neuroimage.2017.07.015 }
$$

55-Girard, G., Whittingstall, K., Deriche, R., Descoteaux, M., 2014. Towards quantitative connectivity analysis: reducing tractography biases. Neurolmage 98, 266-278. https://doi.org/10.1016/j.neuroimage.2014.04.074

56-Glasser, M.F., Sotiropoulos, S.N., Wilson, J.A., Coalson, T.S., Fischl, B., Andersson, J.L., Xu, J., Jbabdi, S., Webster, M., Polimeni, J.R., Van Essen, D.C., Jenkinson, M., 2013. The minimal preprocessing pipelines for the Human Connectome Project. Neurolmage 80, 105-124. https://doi.org/10.1016/j.neuroimage.2013.04.127

57-Goga, C., Türe, U., 2015. The anatomy of Meyer's loop revisited: changing the anatomical paradigm of the temporal loop based on evidence from fiber microdissection. J. Neurosurg. 122 , 1253-1262. 
58-Gorgolewski, K.J., Auer, T., Calhoun, V.D., Craddock, R.C., Das, S., Duff, E.P., Flandin, G., Ghosh, S.S., Glatard, T., Halchenko, Y.O., Handwerker, D.A., Hanke, M., Keator, D., Li, X., Michael, Z., Maumet, C., Nichols, B.N., Nichols, T.E., Pellman, J., Poline, J.-B.B., Rokem, A., Schaefer, G., Sochat, V., Triplett, W., Turner, J.A., Varoquaux, G., Poldrack, R.A., 2016. The brain imaging data structure, a format for organizing and describing outputs of neuroimaging experiments. Sci. Data 3, 1-9. https://doi.org/10.1038/sdata.2016.44

59-Gu, X., Eklund, A., Knutsson, H., 2017. Repeated Tractography of a Single Subject: How High Is the Variance?, in: Schultz, T., Özarslan, E., Hotz, I. (Eds.), Modeling, Analysis, and Visualization of Anisotropy, Mathematics and Visualization. Springer International Publishing, Cham, pp. 331-354. https://doi.org/10.1007/978-3-319-61358-1_14

60-Hansen, C.B., Yang, Q., Lyu, I., Rheault, F., Kerley, C., Chandio, B.Q., Fadnavis, S., Williams, O., Shafer, A.T., Resnick, S.M., Zald, D.H., Cutting, L., Taylor, W.D., Boyd, B., Garyfallidis, E., Anderson, A.W., Descoteaux, M., Landman, B.A., Schilling, K.G., 2020. Pandora: 4-D white matter bundle population-based atlases derived from diffusion MRI fiber tractography. bioRxiv 2020.06.12.148999. https://doi.org/10.1101/2020.06.12.148999

61-Hau, J., Sarubbo, S., Houde, J.C., Corsini, F., Girard, G., Deledalle, C., Crivello, F., Zago, L., Mellet, E., Jobard, G., Joliot, M., Mazoyer, B., Tzourio-Mazoyer, N., Descoteaux, M., Petit, L., 2017. Revisiting the human uncinate fasciculus, its subcomponents and asymmetries with stem-based tractography and microdissection validation. Brain Struct. Funct. 222, 1645-1662. https://doi.org/10.1007/s00429-016-1298-6

62-Heilbronner, S.R., Haber, S.N., 2014. Frontal Cortical and Subcortical Projections Provide a Basis for Segmenting the Cingulum Bundle: Implications for Neuroimaging and Psychiatric Disorders. J. Neurosci. 34, 10041-10054.

63-Herbet, G., Zemmoura, I., Duffau, H., 2018. Functional Anatomy of the Inferior Longitudinal Fasciculus: From Historical Reports to Current Hypotheses. Front. Neuroanat. 12. https://doi.org/10.3389/fnana.2018.00077 
1025

1026

1027

1028

1029

1030

1031

1032

1033

1034

1035

1036

1037

1038

1039

1040

1041

1042

1043

1044

1045

1046

1047

1048

1049

1050

1051

1052

1053

1054

1055

1056

64-Hofer, S., Karaus, A., Frahm, J., 2010. Reconstruction and dissection of the entire human visual pathway using diffusion tensor MRI. Front. Neuroanat. 4. https://doi.org/10.3389/fnana.2010.00015

65-Hong, X., Zheng, L., Rajan, A., Ding, M., 2019. Role of superior longitudinal fasciculus in visual spatial attention. J. Vis. 19, 320-320. https://doi.org/10.1167/19.10.320

66-Jang, S.H., Seo, J.P., 2015. Differences of the medial lemniscus and spinothalamic tract according to the cortical termination areas: A diffusion tensor tractography study. Somatosens. Mot. Res. 32, 67-71. https://doi.org/10.3109/08990220.2014.966899

67-Jenkinson, M., Bannister, P., Brady, M., Smith, S., 2002. Improved optimization for the robust and accurate linear registration and motion correction of brain images. Neurolmage 17, 825-841. https://doi.org/10.1016/s10538119(02)91132-8

68-Jenkinson, M., Beckmann, C.F., Behrens, T.E.J., Woolrich, M.W., Smith, S.M., 2012.

FSL.

Neurolmage

62 ,

782-790.

https://doi.org/10.1016/j.neuroimage.2011.09.015

69-Jeong, J.-W., Asano, E., Yeh, F.-C., Chugani, D.C., Chugani, H.T., 2013. Independent component analysis tractography combined with a ball-stick model to isolate intravoxel crossing fibers of the corticospinal tracts in clinical diffusion MRI. Magn. Reson. Med. 70, 441-453. https://doi.org/10.1002/mrm.24487

70-Jeurissen, B., Tournier, J.-D.D., Dhollander, T., Connelly, A., Sijbers, J., 2014. Multi-tissue constrained spherical deconvolution for improved analysis of multishell diffusion MRI data. Neurolmage 103, 411-426. https://doi.org/10.1016/j.neuroimage.2014.07.061

71-Jhaveri, M.D., Salzman, K.L., Ross, J.S., Moore, K.R., Osborn, A.G., Ho, C.Y., 2018. Middle Cerebellar Peduncle Lesion(s), in: Jhaveri, M.D., Salzman, K.L., Ross, J.S., Moore, K.R., Osborn, A.G., Ho, C.Y. (Eds.), Expertddx: Brain and Spine (Second Edition), ExpertDDx. Elsevier, pp. 518-521. https://doi.org/10.1016/B978-0-323-44308-1.50200-2

72-Jitsuishi, T., Hirono, S., Yamamoto, T., Kitajo, K., Iwadate, Y., Yamaguchi, A., 2020. White matter dissection and structural connectivity of the human vertical 
occipital fasciculus to link vision-associated brain cortex. Sci. Rep. 10, 820. https://doi.org/10.1038/s41598-020-57837-7

73-Johansen-Berg, H., Behrens, T.E.J., 2006. Just pretty pictures? What diffusion tractography can add in clinical neuroscience. Curr. Opin. Neurol. 19, 379-385. https://doi.org/10.1097/01.wco.0000236618.82086.01

74-Jones, D.K., Christiansen, K.F., Chapman, R.J., Aggleton, J.P., 2013. Distinct subdivisions of the cingulum bundle revealed by diffusion MRI fibre tracking: implications for neuropsychological investigations. Neuropsychologia 51, 6778. https://doi.org/10.1016/j.neuropsychologia.2012.11.018

75-kakou, M., Kouakou, F., Nâ ${ }^{\mathrm{TM}}$ dri Oka, D., Mbende, A.S., Peltier, J., Velut, S., 2017. Microanatomy of Thalamic Radiations. Int. J. Hum. Anat. 1, 28-37. https://doi.org/10.14302/issn.2577-2279.ijha-17-1719

76-Kalyvas, A., Koutsarnakis, C., Komaitis, S., Karavasilis, E., Christidi, F., Skandalakis, G.P., Liouta, E., Papakonstantinou, O., Kelekis, N., Duffau, H., Stranjalis, G., 2020. Mapping the human middle longitudinal fasciculus through a focused anatomo-imaging study: shifting the paradigm of its segmentation and connectivity pattern. Brain Struct. Funct. 225, 85-119. https://doi.org/10.1007/s00429-019-01987-6

77-Kikinis, Z., Fitzsimmons, J., Dunn, C., Vu, M.-A., Makris, N., Bouix, S., Goldstein, J.M., Mesholam-Gately, R.I., Petryshen, T., del Re, E.C., Wojcik, J., Seidman, L.J., Kubicki, M., 2015. Anterior Commissural White Matter Fiber Abnormalities in First-Episode Psychosis: A Tractography Study. Schizophr. Res. 162, 29-34. https://doi.org/10.1016/j.schres.2015.01.037

78-Kreilkamp, B.A.K., Lisanti, L., Glenn, G.R., Wieshmann, U.C., Das, K., Marson, A.G., Keller, S.S., 2019. Comparison of manual and automated fiber quantification tractography in patients with temporal lobe epilepsy. Neurolmage Clin. 24, 102024. https://doi.org/10.1016/j.nicl.2019.102024

79-Kurki, T.J.I., Laalo, J.P., Oksaranta, O.M., 2013. Diffusion tensor tractography of the uncinate fasciculus: Pitfalls in quantitative analysis due to traumatic volume changes. J. Magn. Reson. Imaging 38, 46-53. https://doi.org/10.1002/jmri.23901 
80-Kwon, H.G., Hong, J.H., Hong, C.P., Lee, D.H., Ahn, S.H., Jang, S.H., 2011. Dentatorubrothalamic tract in human brain: diffusion tensor tractography study. Neuroradiology 53, 787-791. https://doi.org/10.1007/s00234-011-0878-7 81-La Corte, E., Eldahaby, D., Greco, E., Aquino, D., Bertolini, G., Levi, V., Ottenhausen, M., Demichelis, G., Romito, L.M., Acerbi, F., Broggi, M., Schiariti, M.P., Ferroli, P., Bruzzone, M.G., Serrao, G., 2021. The Frontal Aslant Tract: A Systematic Review for Neurosurgical Applications. Front. Neurol. 12. https://doi.org/10.3389/fneur.2021.641586

82-Larkman, D.J., Hajnal, J.V., Herlihy, A.H., Coutts, G.A., Young, I.R., Ehnholm, G., 2001. Use of multicoil arrays for separation of signal from multiple slices simultaneously excited. J. Magn. Reson. Imaging JMRI 13, 313-317. https://doi.org/10.1002/1522-2586(200102)13:2<313::aid-jmri1045>3.0.co;2-w 83-Latini, F., Mårtensson, J., Larsson, E.-M., Fredrikson, M., Åhs, F., Hjortberg, M., Aldskogius, H., Ryttlefors, M., 2017. Segmentation of the inferior longitudinal fasciculus in the human brain: A white matter dissection and diffusion tensor tractography study. Brain Res. 1675, 102-115. https://doi.org/10.1016/j.brainres.2017.09.005

84-Leitner, Y., Travis, K.E., Ben-Shachar, M., Yeom, K.W., Feldman, H.M., 2015. Tract Profiles of the Cerebellar White Matter Pathways in Children and Adolescents. The Cerebellum 14, 613-623. https://doi.org/10.1007/s12311015-0652-1

85-Liacu, D., Idy-Peretti, I., Ducreux, D., Bouilleret, V., De Marco, G., 2012. Diffusion Tensor Imaging Tractography Parameters of Limbic System Bundles in Temporal Lobe Epilepsy Patients. J Magn Reson Imaging 36, 561-568. https://doi.org/10.1002/jmri.23678

86-Lim, J.C., Phal, P.M., Desmond, P.M., Nichols, A.D., Kokkinos, C., DaneshMeyer, H.V., Kaye, A.H., Moffat, B.A., 2015. Probabilistic MRI Tractography of the Optic Radiation Using Constrained Spherical Deconvolution: A Feasibility Study. PLOS ONE 10 , e0118948-e0118948. https://doi.org/10.1371/journal.pone.0118948 
87-Lingford-Hughes, A., Kalk, N., 2012. 2 - Clinical neuroanatomy, in: Wright, P., Stern, J., Phelan, M. (Eds.), Core Psychiatry (Third Edition). W.B. Saunders, Oxford, pp. 13-34. https://doi.org/10.1016/B978-0-7020-3397-1.00002-1 compressed sensing for rapid MR imaging. Magn. Reson. Med. 58, 1182-1195. https://doi.org/10.1002/mrm.21391

89-Lustig, M., Donoho, D.L., Santos, J.M., Pauly, J.M., 2007b. Compressed sensing MRI, in: leee Signal Processing Magazine.

90-Lyness, R.C., Alvarez, I., Sereno, M.I., MacSweeney, M., 2014. Microstructural differences in the thalamus and thalamic radiations in the congenitally deaf. Neurolmage 100, 347-357. https://doi.org/10.1016/j.neuroimage.2014.05.077

91-Madhavan, K.M., McQueeny, T., Howe, S.R., Shear, P., Szaflarski, J., 2014. Superior Longitudinal Fasciculus and Language Functioning in Healthy Aging. Brain Res. 1562, 11-22. https://doi.org/10.1016/j.brainres.2014.03.012 92-Maffei, C., Lee, C., Planich, M., Ramprasad, M., Ravi, N., Trainor, D., Urban, Z., Kim, M., Jones, R.J., Henin, A., Hofmann, S.G., Pizzagalli, D.A., Auerbach, R.P., Gabrieli, J.D.E., Whitfield-Gabrieli, S., Greve, D.N., Haber, S.N., Yendiki, A., 2021. Using diffusion MRI data acquired with ultra-high gradients to improve tractography in routine-quality data. https://doi.org/10.1101/2021.06.28.450265 93-Maffei, C., Sarubbo, S., Jovicich, J., 2019a. A Missing Connection: A Review of the Macrostructural Anatomy and Tractography of the Acoustic Radiation. Front. Neuroanat. 0. https://doi.org/10.3389/fnana.2019.00027

94-Maffei, C., Sarubbo, S., Jovicich, J., 2019b. Diffusion-based tractography atlas of the human acoustic radiation. Sci. Rep. 9, 4046. https://doi.org/10.1038/s41598-019-40666-8 95-Maier-Hein, K.H., Neher, P.F., Houde, J.-C., Côté, M.-A., Garyfallidis, E., Zhong, J., Chamberland, M., Yeh, F.-C., Lin, Y.-C., Ji, Q., Reddick, W.E., Glass, J.O., Chen, D.Q., Feng, Y., Gao, C., Wu, Y., Ma, J., He, R., Li, Q., Westin, C.-F., Deslauriers-Gauthier, S., González, J.O.O., Paquette, M., St-Jean, S., Girard, G., Rheault, F., Sidhu, J., Tax, C.M.W., Guo, F., Mesri, H.Y., Dávid, S., Froeling, M., Heemskerk, A.M., Leemans, A., Boré, A., Pinsard, B., Bedetti, C., Desrosiers, M., Brambati, S., Doyon, J., Sarica, A., Vasta, R., Cerasa, A., 
Quattrone, A., Yeatman, J., Khan, A.R., Hodges, W., Alexander, S., J.-P., Cetingul, H.E., Odry, B.L., Mailhe, B., Nadar, M.S., Pizzagalli, F., Prasad, G., Villalon-Reina, J.E., Galvis, J., Thompson, P.M., Requejo, F.D.S., Laguna, P.L., Lacerda, L.M., Barrett, R., Dell'Acqua, F., Catani, M., Petit, L., Caruyer, E., Daducci, A., Dyrby, T.B., Holland-Letz, T., Hilgetag, C.C., Stieltjes, B., Descoteaux, M., 2017. The challenge of mapping the human connectome based tractography.

Nat.

Commun.

8 ,

1349. https://doi.org/10.1038/s41467-017-01285-X

D.N., 2009. Delineation of the middle longitudinal fascicle in humans: a quantitative, in vivo, DT-MRI study. Cereb. Cortex N. Y. N 1991 19, 777-785. https://doi.org/10.1093/cercor/bhn124

97-Makris, N., Zhu, A., Papadimitriou, G.M., Mouradian, P., Ng, I., Scaccianoce, E., cortical connections of the human middle longitudinal fascicle in subject-specific, probabilistic, and stereotaxic Talairach spaces. Brain Imaging Behav. 11, 12581277. https://doi.org/10.1007/s11682-016-9589-3

98-Maldonado, I.L., de Champfleur, N.M., Velut, S., Destrieux, C., Zemmoura, I., Duffau, H., 2013. Evidence of a middle longitudinal fasciculus in the human brain from fiber dissection. J. Anat. 223, 38-45. https://doi.org/10.1111/joa.12055 99-Martínez-Heras, E., Varriano, F., Prčkovska, V., Laredo, C., Andorrà, M., Martínez-Lapiscina, E.H., Calvo, A., Lampert, E., Villoslada, P., Saiz, A., PratsGalino, A., Llufriu, S., 2015. Improved Framework for Tractography Reconstruction of the Optic Radiation. PloS One 10, e0137064-e0137064. https://doi.org/10.1371/journal.pone.0137064 
1181 101- Mehra, D., Moshirfar, M., 2021. Neuroanatomy, Optic Tract, in:

StatPearls. StatPearls Publishing, Treasure Island (FL).

102- Menjot de Champfleur, N., Lima Maldonado, I., Moritz-Gasser, S.,

Machi, P., Le Bars, E., Bonafé, A., Duffau, H., 2013. Middle longitudinal

fasciculus delineation within language pathways: A diffusion tensor imaging

study in human. Eur. J. Radiol., Special Section: Imaging of the Peripheral

Nervous System 82, 151-157. https://doi.org/10.1016/j.ejrad.2012.05.034

Aggleton, J.P., O'Sullivan, M.J., 2012. Cingulum Microstructure Predicts

Cognitive Control in Older Age and Mild Cognitive Impairment. J. Neurosci. 32, 17612-17619.

104- Meynert, T., 1888. Psychiatrie clinique des maladies du cerveau antérieur. Psychiatr. Clin. Mal. Cerveau Antér. 294-294.

105- Miller, K.L., Alfaro-Almagro, F., Bangerter, N.K., Thomas, D.L., Yacoub,

E., Xu, J., Bartsch, A.J., Jbabdi, S., Sotiropoulos, S.N., Andersson, J.L.,

Multimodal population brain imaging in the UK Biobank prospective epidemiological study. Nat. Neurosci. 19, 1523-1536. https://doi.org/10.1038/nn.4393

106- Moeller, S., Yacoub, E., Olman, C.A., Auerbach, E., Strupp, J., Harel, N., Uğurbil, K., 2010. Multiband multislice GE-EPI at 7 tesla, with 16-fold acceleration using partial parallel imaging with application to high spatial and temporal whole-brain fMRI. Magn. Reson. Med. 63, 1144-1153. https://doi.org/10.1002/mrm.22361

107- Mollink, J., van Baarsen, K.M., Dederen, P.J.W.C., Foxley, S., Miller, K.L., Jbabdi, S., Slump, C.H., Grotenhuis, J.A., Kleinnijenhuis, M., van Cappellen van Walsum, A.M., 2016. Dentatorubrothalamic tract localization with postmortem MR diffusion tractography compared to histological 3D reconstruction. Brain Struct. Funct. 221, 3487-3501. https://doi.org/10.1007/s00429-015-1115-7 
108- Mori, S., Crain, B.J., Chacko, V.P., van Zijl, P.C., 1999. Threedimensional tracking of axonal projections in the brain by magnetic resonance imaging. Ann. Neurol. 45, 265-269. https://doi.org/10.1002/15318249(199902)45:2<265::aid-ana21>3.0.co;2-3

109- Mori, S., Oishi, K., Faria, A.V., 2009. White matter atlases based on

diffusion tensor imaging. Curr. Opin. Neurol. 22, 362-369. https://doi.org/10.1097/WCO.0b013e32832d954b

110- Mori, S., Oishi, K., Jiang, H., Jiang, L., Li, X., Akhter, K., Hua, K., Faria, white matter atlas based on diffusion tensor imaging in an ICBM template.

Neurolmage 40, 570-582. https://doi.org/10.1016/j.neuroimage.2007.12.035

112- Nachtergaele, P., Radwan, A., Swinnen, S., Decramer, T.,

Uytterhoeven, M., Sunaert, S., van Loon, J., Theys, T., 2019. The https://doi.org/10.3171/2018.11.JNS18679

113- Nazem-Zadeh, M.-R., Saksena, S., Babajani-Fermi, A., Jiang, Q., Soltanian-Zadeh, H., Rosenblum, M., Mikkelsen, T., Jain, R., 2012.

Segmentation of corpus callosum using diffusion tensor imaging: validation in patients with glioblastoma. BMC Med. Imaging 12, 10. https://doi.org/10.1186/1471-2342-12-10

114- Niida, R., Yamagata, B., Niida, A., Uechi, A., Matsuda, H., Mimura, M., 2018. Aberrant Anterior Thalamic Radiation Structure in Bipolar Disorder: A Diffusion Tensor Tractography Study. Front. Psychiatry 9. https://doi.org/10.3389/fpsyt.2018.00522

115- Nowacki, A., Schlaier, J., Debove, I., Pollo, C., 2018. Validation of diffusion tensor imaging tractography to visualize the dentatorubrothalamic tract 
for surgical planning. J. Neurosurg. 130, 99-108.

116- Ohoshi, Y., Takahashi, S., Yamada, S., Ishida, T., Tsuda, K., Tsuji, T.,

Terada, M., Shinosaki, K., Ukai, S., 2019. Microstructural abnormalities in callosal fibers and their relationship with cognitive function in schizophrenia: $A$ tract-specific analysis study. Brain Behav.

9, e01357. https://doi.org/10.1002/brb3.1357

Panesar, S.S., Abhinav, K., Yeh, F.-C., Jacquesson, T., Collins, M.,

118- Panesar, S.S., Yeh, F.-C., Jacquesson, T., Hula, W., FernandezMiranda, J.C., 2018. A Quantitative Tractography Study Into the Connectivity,

Segmentation and Laterality of the Human Inferior Longitudinal Fasciculus.

119- Pascalau, R., Popa Stănilă, R., Sfrângeu, S., Szabo, B., 2018. Anatomy of the Limbic White Matter Tracts as Revealed by Fiber Dissection and Tractography. World Neurosurg. 113, e672-e689. https://doi.org/10.1016/j.wneu.2018.02.121

1266 121- Peltier, J., Verclytte, S., Delmaire, C., Pruvo, J.-P., Havet, E., Le Gars, 1267 D., 2011. Microsurgical Anatomy of the Anterior Commissure: Correlations With

120- Pascual-Diaz, S., Varriano, F., Pineda, J., Prats-Galino, A., 2020. Structural characterization of the Extended Frontal Aslant Tract trajectory: A MLvalidated laterality study in 3T and 7T. Neurolmage 222, 117260. https://doi.org/10.1016/j.neuroimage.2020.117260

Diffusion Tensor Imaging Fiber Tracking and Clinical Relevance. Oper. Neurosurg. 69 , ons241-ons247. https://doi.org/10.1227/NEU.0b013e31821bc822

122- Peng, H., Cirstea, C.M., Kaufman, C.L., Frey, S.H., 2019a. Microstructural integrity of corticospinal and medial lemniscus tracts: insights from diffusion tensor tractography of right-hand amputees. J. Neurophysiol. 122, 316-324. https://doi.org/10.1152/jn.00316.2018 
123- Peng, H., Cirstea, C.M., Kaufman, C.L., Frey, S.H., 2019b.

Microstructural integrity of corticospinal and medial lemniscus tracts: insights

124- Phillips, O., Sanchez-Castaneda, C., Elifani, F., Maglione, V., Di Pardo,

A., Caltagirone, C., Squitieri, F., Sabatini, U., Di Paola, M., 2013. Tractography

of the Corpus Callosum in Huntington's Disease. PLoS ONE 8.

https://doi.org/10.1371/journal.pone.0073280

125- Portegies, J.M., Fick, R.H.J., Sanguinetti, G.R., Meesters, S.P.L., Girard,

G., Duits, R., 2015. Improving Fiber Alignment in HARDI by Combining

126- Radwan, A., Nachtergaele, P., Swinnen, S., Decramer, T.,

Uytterhoeven, M., van Loon, J., Theys, T., Sunaert, S., 2019. The temporoinsular projection system: a multisubject fiber tractography study using connectome diffusion da, in: Proc. Intl. Soc. Mag. Reson. Med Intl. 27. Leiden, NL, pp. 34-34. https://doi.org/10.13140/RG.2.2.20513.76642

Re, T.J., Levman, J., Lim, A.R., Righini, A., Grant, P.E., Takahashi, E., 2017. High-angular resolution diffusion imaging tractography of cerebellar pathways from newborns to young adults. Brain Behav. 7, e00589. https://doi.org/10.1002/brb3.589

128- Rheault, F., De Benedictis, A., Daducci, A., Maffei, C., Tax, C.M.W., Chamberland, M., Barakovic, M., Goyette, N., Poulin, P., Chenot, Q., Panesar, S.S., Sarubbo, S., Petit, L., Descoteaux, M., 2020. Tractostorm: The what, why, and how of tractography dissection reproducibility. Hum. Brain Mapp. 41, 18591874. https://doi.org/10.1002/hbm.24917

129- Rheault, F., St-Onge, E., Sidhu, J., Maier-Hein, K., Tzourio-Mazoyer, N., Petit, L., Descoteaux, M., 2019. Bundle-specific tractography with incorporated anatomical and orientational priors. Neurolmage 186, 382-398. https://doi.org/10.1016/j.neuroimage.2018.11.018 
130- Salamon, N., Sicotte, N., Drain, A., Frew, A., Alger, J.R., Jen, J., Perlman, S., Salamon, G., 2007. White matter fiber tractography and color mapping of the normal human cerebellum with diffusion tensor imaging. J. Neuroradiol. 34, 115-128. https://doi.org/10.1016/j.neurad.2007.03.002 131- Schilling, K.G., Rheault, F., Petit, L., Hansen, C.B., Nath, V., Yeh, F.-C., Girard, G., Barakovic, M., Rafael-Patino, J., Yu, T., Fischi-Gomez, E., Pizzolato, M., Ocampo-Pineda, M., Schiavi, S., Canales-Rodríguez, E.J., Daducci, A., Granziera, C., Innocenti, G., Thiran, J.-P., Mancini, L., Wastling, S., Cocozza, S., Petracca, M., Pontillo, G., Mancini, M., Vos, S.B., Vakharia, V.N., Duncan, J.S., Melero, H., Manzanedo, L., Sanz-Morales, E., Peña-Melián, Á., Calamante, F., Attyé, A., Cabeen, R.P., Korobova, L., Toga, A.W., Vijayakumari, A.A., Parker, D., Verma, R., Radwan, A., Sunaert, S., Emsell, L., Luca, A.D., Leemans, A., Bajada, C.J., Haroon, H., Azadbakht, H., Chamberland, M., Genc, S., Tax, C.M.W., Yeh, P.-H., Srikanchana, R., Mcknight, C., Yang, J.Y.-M., Chen, J., Kelly, C.E., Yeh, C.-H., Cochereau, J., Maller, J.J., Welton, T., Almairac, F., Seunarine, K.K., Clark, C.A., Zhang, F., Makris, N., Golby, A., Rathi, Y., O’Donnell, L.J., Xia, Y., Aydogan, D.B., Shi, Y., Fernandes, F.G., Raemaekers, M., Warrington, S., Michielse, S., Ramírez-Manzanares, A., Concha, L., Aranda, R., Meraz, M.R., Lerma-Usabiaga, G., Roitman, L., Fekonja, L.S., Calarco, N., Joseph, M., Nakua, H., Voineskos, A.N., Karan, P., Grenier, G., Legarreta, J.H., Adluru, N., Nair, V.A., Prabhakaran, V., Alexander, A.L., Kamagata, K., Saito, Y., Uchida, W., Andica, C., Masahiro, A., Bayrak, R.G., Wheeler-Kingshott, C.A.M.G., D’Angelo, E., Palesi, F., Savini, G., Rolandi, N., Guevara, P., Houenou, J., López-López, N., Mangin, J.-F., Poupon, C., Román, C., Vázquez, A., Maffei, C., Arantes, M., Andrade, J.P., Silva, S.M., Raja, R., Calhoun, V.D., Caverzasi, E., Sacco, S., Lauricella, M., Pestilli, F., Bullock, D., Zhan, Y., Brignoni-Perez, E., Lebel, C., Reynolds, J.E., Nestrasil, I., Labounek, R., Lenglet, C., Paulson, A., Aulicka, S., Heilbronner, S., Heuer, K., Anderson, A.W., Landman, B.A., Descoteaux, M., 2020. Tractography dissection variability: what happens when 42 groups dissect 14 white matter bundles on the same dataset? bioRxiv 2020.10.07.321083. https://doi.org/10.1101/2020.10.07.321083 
132- Schilling, K.G., Tax, C.M., Rheault, F., Hansen, C.B., Yang, Q., Yeh, F.C., Cai, L.Y., Anderson, A.W., Landman, B.A., 2021. Fiber tractography bundle segmentation depends on scanner effects, vendor effects, acquisition resolution, diffusion sampling scheme, diffusion sensitization, and bundle $\begin{array}{lll}\text { segmentation } \quad \text { workflow. } & \text { bioRxiv } 21.03 .17 .435872 .\end{array}$ https://doi.org/10.1101/2021.03.17.435872

133- Schurr, R., Filo, S., Mezer, A.A., 2019. Tractography delineation of the vertical occipital fasciculus using quantitative T1 mapping. Neurolmage 202, 116121-116121. https://doi.org/10.1016/j.neuroimage.2019.116121

134- Scilpy documentation [WWW Document], 2021. URL https://scilpy.readthedocs.io/en/latest/ (accessed 2.15.21).

135- Seltzer, B., Pandya, D.N., 1984. Further observations on parietotemporal connections in the rhesus monkey. Exp. Brain Res. 55, 301-312. https://doi.org/10.1007/BF00237280

136- Skare, S., Bammer, R., 2009. Jacobian weighting of distortion corrected EPI data [WWW Document]. URL https://www.semanticscholar.org/paper/Jacobian-weighting-of-distortioncorrected-EPI-data-Skare-

Bammer/7ed01244fd7c4ab273656d40b8b76fcbdc4bee7d (accessed 6.26.21). 137- Smith, R.E., Tournier, J.-D., Calamante, F., Connelly, A., 2015. SIFT2: Enabling dense quantitative assessment of brain white matter connectivity using streamlines tractography. Neurolmage 119, 338-351. https://doi.org/10.1016/j.neuroimage.2015.06.092

138- Smith, R.E., Tournier, J.-D., Calamante, F., Connelly, A., 2013. SIFT: Spherical-deconvolution informed filtering of tractograms. Neurolmage 67, 298312. https://doi.org/10.1016/j.neuroimage.2012.11.049

139- Smith, S.M., Jenkinson, M., Woolrich, M.W., Beckmann, C.F., Behrens, T.E.J., Johansen-berg, H., Bannister, P.R., Luca, M.D., Drobnjak, I., Flitney, D.E., Niazy, R.K., Saunders, J., Vickers, J., Zhang, Y., Stefano, N.D., Brady, J.M., Matthews, P.M., 2004. Advances in functional and structural MR image analysis and implementation as FSL 23, 208-219. https://doi.org/10.1016/j.neuroimage.2004.07.051 
Soares, J.M., Marques, P., Alves, V., Sousa, N., 2013. A hitchhiker's guide to diffusion tensor imaging. Front. Neurosci. 7, 1-14. https://doi.org/10.3389/fnins.2013.00031

141- Stieltjes, B., Kaufmann, W.E., van Zijl, P.C.M., Fredericksen, K.,

Pearlson, G.D., Solaiyappan, M., Mori, S., 2001. Diffusion Tensor Imaging and

Axonal Tracking in the Human Brainstem. Neurolmage 14, 723-735.

142- $\quad$ Strange, B.A., Witter, M.P., Lein, E.S., Moser, E.I., 2014. Functional

143- Thiebaut de Schotten, M., ffytche, D.H., Bizzi, A., Dell'Acqua, F., Allin, M., Walshe, M., Murray, R., Williams, S.C., Murphy, D.G.M., Catani, M., 2011.

Atlasing location, asymmetry and inter-subject variability of white matter tracts in the human brain with MR diffusion tractography. Neurolmage 54, 49-59. https://doi.org/10.1016/j.neuroimage.2010.07.055

144- Toselli, B., Tortora, D., Severino, M., Arnulfo, G., Canessa, A., Morana,

G., Rossi, A., Fato, M.M., 2017. Improvement in White Matter Tract

Review. Front. Pediatr. 5, 182. https://doi.org/10.3389/fped.2017.00182

146- Tournier, J.-D., Calamante, F., Connelly, A., 2010. Improved probabilistic streamlines tractography by 2nd order integration over fibre orientation distributions. Proc Intl Soc Mag Reson Med ISMRM 18.

1400 148- Tournier, J.-D., Smith, R.E., Raffelt, D.A., Tabbara, R., Dhollander, T., 1401 Pietsch, M., Christiaens, D., Jeurissen, B., Yeh, C.-H., Connelly, A., 2019. 
processing and visualisation. Neurolmage 202, 116137-116137. https://doi.org/10.1016/j.neuroimage.2019.116137

149- Tsao, H., Pannek, K., Boyd, R.N., Rose, S.E., 2015. Changes in the integrity of thalamocortical connections are associated with sensorimotor deficits in children with congenital hemiplegia. Brain Struct. Funct. 220, 307318. https://doi.org/10.1007/s00429-013-0656-x

150- van Baarsen, K.M., Kleinnijenhuis, M., Jbabdi, S., Sotiropoulos, S.N., Grotenhuis, J.A., van Cappellen van Walsum, A.M., 2016. A probabilistic atlas of the cerebellar white matter. Neurolmage 124, 724-732. https://doi.org/10.1016/j.neuroimage.2015.09.014

151- Van Essen, D.C., Ugurbil, K., Auerbach, E., Barch, D., Behrens, T.E.J., Bucholz, R., Chang, A., Chen, L., Corbetta, M., Curtiss, S.W., Della Penna, S., Feinberg, D., Glasser, M.F., Harel, N., Heath, A.C., Larson-Prior, L., Marcus, D., Michalareas, G., Moeller, S., Oostenveld, R., Petersen, S.E., Prior, F., Schlaggar, B.L., Smith, S.M., Snyder, A.Z., Xu, J., Yacoub, E., WU-Minn HCP Consortium, 2012. The Human Connectome Project: a data acquisition perspective. Neurolmage 62 ,

2222-2231. https://doi.org/10.1016/j.neuroimage.2012.02.018 152- Van Hecke, W., Emsell, L., Sunaert, S. (Eds.), 2016. Diffusion Tensor Imaging: A Practical Handbook. Springer-Verlag, New York. https://doi.org/10.1007/978-1-4939-3118-7

153- van Meer, N., Houtman, A.C., Van Schuerbeek, P., Vanderhasselt, T., Milleret, C., ten Tusscher, M.P., 2016. Interhemispheric Connections between the Primary Visual Cortical Areas via the Anterior Commissure in Human $\begin{array}{lllll}\text { Callosal Agenesis. } & \text { Front. }\end{array}$ https://doi.org/10.3389/fnsys.2016.00101

154- Veraart, J., Fieremans, E., Novikov, D.S., 2016. Diffusion MRI Noise Mapping Using Random Matrix Theory 1593, 1582-1593. https://doi.org/10.1002/mrm.26059

155- Verhoeven, J.S., Sage, C.A., Leemans, A., Van Hecke, W., Callaert, D., Peeters, R., De Cock, P., Lagae, L., Sunaert, S., 2010. Construction of a stereotaxic DTI atlas with full diffusion tensor information for studying white 
matter maturation from childhood to adolescence using tractography-based segmentations. Hum. Brain Mapp. 31, 470-486. https://doi.org/10.1002/hbm.20880

156- Voogd, J., 2004. CHAPTER 11 - Cerebellum and Precerebellar Nuclei, in: Paxinos, G., Mai, J.K. (Eds.), The Human Nervous System (Second Edition). Academic Press, San Diego, pp. 321-392. https://doi.org/10.1016/B978012547626-3/50012-0

157- Wakana, S., Caprihan, A., Panzenboeck, M.M., Fallon, J.H., Perry, M., Gollub, R.L., Hua, K., Zhang, J., Jiang, H., Dubey, P., Blitz, A., Van Zijl, P., Mori, S., 2007. Reproducibility of Quantitative Tractography Methods Applied to Cerebral White Matter.

158- Wakana, S., Jiang, H., Nagae-Poetscher, L.M., Zijl, P.C.M. van, Mori, S., 2004. Fiber Tract-based Atlas of Human White Matter Anatomy. Radiology. https://doi.org/10.1148/radiol.2301021640

159- Wang, X., Pathak, S., Stefaneanu, L., Yeh, F.-C., Li, S., FernandezMiranda, J.C., 2016. Subcomponents and connectivity of the superior longitudinal fasciculus in the human brain. Brain Struct. Funct. 221, 2075-2092. https://doi.org/10.1007/s00429-015-1028-5

160- Wang, Y., Fernández-Miranda, J.C., Verstynen, T., Pathak, S., Schneider, W., Yeh, F.-C., 2013. Rethinking the Role of the Middle Longitudinal Fascicle in Language and Auditory Pathways. Cereb. Cortex 23, 2347-2356. https://doi.org/10.1093/cercor/bhs225

161- Warrington, S., Bryant, K.L., Khrapitchev, A.A., Sallet, J., CharqueroBallester, M., Douaud, G., Jbabdi, S., Mars, R.B., Sotiropoulos, S.N., 2020. XTRACT - Standardised protocols for automated tractography in the human and $\begin{array}{llll}\text { macaque brain. } & \text { Neurolmage } & 216923 .\end{array}$ https://doi.org/10.1016/j.neuroimage.2020.116923

162- Wasserthal, J., Neher, P., Maier-Hein, K.H., 2018. TractSeg - Fast and accurate white matter tract segmentation. Neurolmage 183, 239-253.

163- Wilde, E.A., Bigler, E.D., Haider, J.M., Chu, Z., Levin, H.S., Li, X., Hunter, J.V., 2006. Vulnerability of the anterior commissure in moderate to 
severe pediatric traumatic brain injury. J. Child Neurol. 21, 769-776. https://doi.org/10.1177/08830738060210090201

164- Wilkins, B., Lee, N., Gajawelli, N., Law, M., Leporé, N., 2015. Fiber estimation and tractography in diffusion MRI: Development of simulated brain images and comparison of multi-fiber analysis methods at clinical b-values. Neurolmage 109, 341-356. https://doi.org/10.1016/j.neuroimage.2014.12.060 165- Wu, W., Rigolo, L., O’Donnell, L.J., Norton, I., Shriver, S., Golby, A.J.,

2012. Visual Pathway Study Using In Vivo Diffusion Tensor Imaging Tractography to Complement Classic Anatomy. Oper. Neurosurg. 70, ons145ons156. https://doi.org/10.1227/NEU.0b013e31822efcae

Wu, Y., Sun, D., Wang, Yong, Wang, Yibao, 2016a. Subcomponents and

Connectivity of the Inferior Fronto-Occipital Fasciculus Revealed by Diffusion

Spectrum Imaging Fiber Tracking. Front. Neuroanat. 10.

167- Wu, Y., Sun, D., Wang, Yong, Wang, Yibao, Ou, S., $2016 \mathrm{~b}$.

168- Xiao, Y., Beriault, S., Pike, G.B., Collins, D.L., 2012. Multicontrast multiecho FLASH MRI for targeting the subthalamic nucleus. Magn. Reson. Imaging 30, 627-640. https://doi.org/10.1016/j.mri.2012.02.006

169- Xiao, Y., Fonov, V., Bériault, S., Al Subaie, F., Chakravarty, M.M., Sadikot, A.F., Pike, G.B., Collins, D.L., 2015. Multi-contrast unbiased MRI atlas of a Parkinson's disease population. Int. J. Comput. Assist. Radiol. Surg. 10, 329-341. https://doi.org/10.1007/s11548-014-1068-y

170- Xiao, Y., Fonov, V., Chakravarty, M.M., Beriault, S., Al Subaie, F., Sadikot, A., Pike, G.B., Bertrand, G., Collins, D.L., 2017. A dataset of multi1496 contrast population-averaged brain MRI atlases of a Parkinson's disease cohort. Data Brief 12, 370-379. https://doi.org/10.1016/j.dib.2017.04.013

171- Yeatman, J.D., Weiner, K.S., Pestilli, F., Rokem, A., Mezer, A., Wandell, B.A., 2014. The vertical occipital fasciculus: A century of controversy resolved by in vivo measurements. Proc. Natl. Acad. Sci. 111, E5214-E5223. 
172- Yeh, F.-C., Panesar, S., Fernandes, D., Meola, A., Yoshino, M., Fernandez-Miranda, J.C., Vettel, J.M., Verstynen, T., 2018. Populationaveraged atlas of the macroscale human structural connectome and its network topology. Neurolmage 178, 57-68. https://doi.org/10.1016/j.neuroimage.2018.05.027

173- Yendiki, A., Panneck, P., Srinivasan, P., Stevens, A., Zöllei, L., Augustinack, J., Wang, R., Salat, D., Ehrlich, S., Behrens, T., Jbabdi, S., Gollub, R., Fischl, B., 2011. Automated Probabilistic Reconstruction of White-Matter Pathways in Health and Disease Using an Atlas of the Underlying Anatomy. Front. Neuroinformatics 5. https://doi.org/10.3389/fninf.2011.00023

174- Younes, K., Hasan, K.M., Kamali, A., McGough, C.E., Keser, Z., Hasan, O., Melicher, T., Kramer, L.A., Schulz, P.E., 2019. Diffusion Tensor Imaging of the Superior Thalamic Radiation and Cerebrospinal Fluid Distribution in Idiopathic Normal Pressure Hydrocephalus. J. Neuroimaging 29, 242-251. https://doi.org/10.1111/jon.12581

175- Zhang, F., Wu, Y., Norton, I., Rathi, Y., Golby, A.J., O’Donnell, L.J., 2019. Test-retest reproducibility of white matter parcellation using diffusion MRI tractography fiber clustering. Hum. Brain Mapp. 40, 3041-3057. https://doi.org/10.1002/hbm.24579

176- Zhang, Y., Zhang, J., Oishi, K., Faria, A.V., Jiang, H., Li, X., Akhter, K., Rosa-Neto, P., Pike, G.B., Evans, A., Toga, A.W., Woods, R., Mazziotta, J.C., Miller, M.I., van Zijl, P.C.M., Mori, S., 2010. Atlas-guided tract reconstruction for automated and comprehensive examination of the white matter anatomy. Neurolmage 52, 1289-301. https://doi.org/10.1016/j.neuroimage.2010.05.049 177- Zheng, Y., Wang, D., Ye, Q., Zou, F., Li, Y., Kwok, S.C., 2020. Diffusion property and functional connectivity of superior longitudinal fasciculus underpin human metacognition. bioRxiv 2020.03.17.994574. https://doi.org/10.1101/2020.03.17.994574 